\title{
Idiopathic glomerular disease : a prospective study of chronic haematuria and nephrotic syndrome
}

Citation for published version (APA):

Nieuwhof, C. M. G. (1999). Idiopathic glomerular disease : a prospective study of chronic haematuria and nephrotic syndrome. [Doctoral Thesis, Maastricht University]. Universiteit Maastricht. https://doi.org/10.26481/dis.19990115cn

Document status and date:

Published: 01/01/1999

DOI:

$10.26481 /$ dis. $19990115 \mathrm{cn}$

Document Version:

Publisher's PDF, also known as Version of record

\section{Please check the document version of this publication:}

- A submitted manuscript is the version of the article upon submission and before peer-review. There can be important differences between the submitted version and the official published version of record.

People interested in the research are advised to contact the author for the final version of the publication, or visit the DOI to the publisher's website.

- The final author version and the galley proof are versions of the publication after peer review.

- The final published version features the final layout of the paper including the volume, issue and page numbers.

Link to publication

\footnotetext{
General rights rights.

- You may freely distribute the URL identifying the publication in the public portal. please follow below link for the End User Agreement:

www.umlib.nl/taverne-license

Take down policy

If you believe that this document breaches copyright please contact us at:

repository@maastrichtuniversity.nl

providing details and we will investigate your claim.
}

Copyright and moral rights for the publications made accessible in the public portal are retained by the authors and/or other copyright owners and it is a condition of accessing publications that users recognise and abide by the legal requirements associated with these

- Users may download and print one copy of any publication from the public portal for the purpose of private study or research.

- You may not further distribute the material or use it for any profit-making activity or commercial gain

If the publication is distributed under the terms of Article $25 \mathrm{fa}$ of the Dutch Copyright Act, indicated by the "Taverne" license above, 


\section{Idiopathic Glomerular Disease}

A prospective study of chronic haematuria and nephrotic syndrome 
Printed by Unigraphic

ISBN $90-5681-042-1$

Cover by Lea Nieuwhof

The studies in this thesis were financially supported by a grant of the Nierstichting Nederland (grant number C 89.929) and by the University Hospital Maastricht. 


\section{IDIOPATHIC GLOMERULAR DISEASE}

A prospective study of chronic haematuria and nephrotic syndrome

\section{PROEFSCHRIFT}

Ter verkrijging van de graad van doctor aan

de Universiteit Maastricht, op gezag van de Rector Magnificus,

Prof. Dr. A.C. Nieuwenhuijzen Kruseman volgens het besluit van het College van Decanen, in het openbaar te verdedigen op vrijdag 15 jamuari 1999 om 16.00 uur

door

Christina Maria Gertrudis Nieuwhof geboren op 9 juni 1964 te Beek 
Promotor:

Co-promotor:

Beoordelingscommissie:
Prof. Dr. P.J.C. van Breda Vriesman

Dr. $\mathbf{K}$ Leunissen

Prof. Dr. J.W. Arends (voorzitter)

Prof. Dr. L.A. van Es (Universiteit van Leiden)

Prof. Dr. P.W. de Leeuw

Prof. Dr. D.W. Slaaf

Prof. Dr. J.J. Weening (Universiteit van Amsterdam) 
aan mijn ouders 



\section{Contents}

Abbreviations $\quad 8$

Introduction 9

Chapter 1: Idiopathic glomerular disease: study of the natural history provides important information for decision-making in treatment of renal disease.

Chapter 2: A prospective study of the natural history of idiopathic non-proteinuric haematuria

Chapter 3: Thin GBM nephropathy: premature glomerular obsollescence is associated with hypertension and late onset renal failure

Chapter 4: Chronicity index and mesangial IgG deposition are risk factors for hypertension and renal failure in early IgA nephropathy

Chapter 5: Natural history of idiopathic membranous glomerulonephritis

Summary

Nederlandse samenvatting

List of publications

Curriculum vitae 


\section{Abbreviations}

ACE
CADI
C5b-9
COLAA3
COLAA4
ERPF
ESRD
FGS
FSGS
GBM
GFR
GN
IgAN
IMGN
NCI-domain
NSAD
PAH
PCR
SLE
TGF- $\beta$

Angiotension-converting-enzyme

Chronic allograft damage index

Complement factor 5b-9, membrane-attackcomplex

Collagen type 4, 03 -chain

Collagen type 4 , $\alpha 4 \times$ chain

Effective renal plasma flow

End-stage renal disease

Focal global glomerulosclerosis

Focal and segmental hyalinosis and sclerosis

Glomerular basement membrane

Glomenuar filtration rate

Glomerulonephritis

IgA nephropathy

Idiopathic membranous glomerulonephritis non-collagenous domain of collagen

Non steroid-anti-inflammatory-drugs

Para-amino-hippuric-acid

Polymerase chain reaction

Systemic lupus erythematosis

Transforming-growth factor $-\beta$ 


\section{Introduction}


This thesis was designed to chart the natural history of idiopathic glomenulonephritis and to identify risk factors for a poor renal prognosis early in the natural history of disease. This was feasible because the patients who were followed in this study were all included shortly after patient identification, thus at an early stage of disease. The patients originated from a prospective regional study on the epidemiology of idiopathic glomerulonephritis executed between 1978-1984. The study outline ruled out a centre-effect by avoiding a concentration of patients with an unfavourable prognosis in academic centres. Financing was not dependent on extemal funding; the Dutch Kidney Foundation enabled follow up and repeat renal biopsies. A long-term follow-up was thus ensured: 12 years median with a range of 9 to 15 years. Sufficient data were available for analysis of the natural history of patients who presented with idiopathic chronic macroscopic or microscopic haematuria and of patients who were biopsied because of idiopathic nephrotic syndrome.

In chapter 1 , which is a review of the literature, we discuss the role of renal biopsies in the diagnosis and management of idiopathic glomerular diseases like IgA nephropathy and membranous glomerullonephritis. As clinical parameters appear insufficient to differentiate between glomerular and urological causes of haematuria. (quantitation of dysmorphic erythrocytes), renal biopsy is required to establish the diagnosis. As further shown in this thesis, it also provides information about prognosis. In membranous glomerulonephritis, renal biopsies enable determination of immunological activity and should be used liberally to guide management decisions, like treatment with immunosuppressive medication or symptomatic and supportive treatment.

In chapter 2 the natural history of idiopathic non-proteinuric non-azotaemic haematuria is presented. Long-term follow-up of these patients showed that IgA mephropathy and thin glomerular basement membrane (GBM) nephropathy are equal risk factors for the development of hypertension. The hypertension in thin GBM nephropathy is probably of renal origin as it appears associated with increased percentages of global glomerulosclerosis. This inevitably lead to the question whether or not thin GBM nephropathy is form fruste of Alport syndrome.

In chapter 3 we show that although thin GBM nephropathy may share some ulltrastructural glomerular characteristics with early Alport syndrome, thin GBM nephropathy differs from Alport in terms of glomerular immunohistochemistry, 
evolution of the glomerular lesions and absence of extrarenal signs of disease. Thin GBM nephropathy shows in half of the patients a familial pattern of inheritance with unexplained renal insufficiency occurring in roughly one quarter of the families. GFR and ERPF determinations in normotensive thin GBM patients show decreased renal function in several elderly patients. A tempting bypothesis links the increased presence of focal global glomerulosclerosis, development of hypertension, abnormal GFR and ERPF determinations and renal insufficiency in older age in this syndrome.

In chapter 4 the natural history of idiopathic IgA nephropathy is outlined. A histological, semi-quantitative, scoring system was developed to assess the activity and chronicity of lesions. This scoring system consist of an activity and a chronicity index and derives from the NIH classification of Lupus nephritis. This modified NIH index, together with immunohistochemical analysis and clinical parameters of disease was. used to determine prognosis in early idiopathic IgA nephropathy. We show that, in the univariate analysis, mesangial $\operatorname{IgG}$ deposition and a higher initial chronicity index were associated with development of hypertension, while development of uraemia was associated with initial proteinuria over $1000 \mathrm{mg} / \mathrm{day}$. High initial proteimuria is related. to a high activity index and to extracapillary crescents, but not to the development of bypertension. Kaplan Meier curves showed that mesangial IgG deposition, initial type of haematuria and a chronicity index higher than 3 had a significant influence on the clinical disease-free interval. In the multivariate analysis, a high initial chronicity index and presence of erythrocyturia as presenting symptom are independent determinants of the natural bistory of disease.

In chapter 5 the natural history of idiopathic membranous glomerulonephritis is described. Patients were included according to strict indication to biopsy, early in the disease history. We show that idiopathic membranous glomerulonephritis presents almost invariably with a proteinuria over 5 grams per day and with stage 1 histology. The chronicity of the proteinuria, and not its sewerity at onset, is a risk factor for renal insufficiency. Repeat renal biopsies are required to differentiate immune-mediated chronic proteinuria from proteinuria due to non-immunological mechanisms. 


\section{Chapter 1.}

Study of the natural history of idiopathic glomerulonephritis provides important information for decision-making in treatment of renal disease. 
In the Netherlands one in 2000 citizens currently needs treatment for end-stage renal disease. An additional 1.3 in 1000 (some 20 000) citizens suffer from renal failure that might necessitate renal replacement therapy in the future. The total number of patients on replacement therapy has increased threefold in the period between 1980 1994; this increase is primarily seen in the population over 65 years of age [1]. Idiopathic glomerular disease still is an important cause of renal insufficiency requiring renal replacement therapy. When the prospective regional epidemiological study of idiopathic glomenular disease started, in the period 1978-1984, idiopathic glomerular disease accounted for some $27 \%$ of mew patients with end-stage renal disease (ESRD) that entered renal replacement programmes. Over the years, diabetic nephropathy has become more important as a cause of renal failure requiring replacement treatment (in the European population the yearly percentage of new patients with end-stage renal disease due to diabetic nephropathy increased from $8 \%$ to $15 \%$ [2]; in 1990 one third of all new patients who entered renal replacement therapies in the United States suffered from diabetic nephropathy [3]). Likewise, hypertensive and renovascular nephropathies are increasingly important in causing end-stage renal disease. Another $19 \%$ of the patients with ESRD had no definitive diagnosis. Still, idiopathic glomenular disease remains the cause of renal insufficiency in 12-21\% of the patients (table 1 and 2) $[2,3]$. Idiopathic glomerular disease usually is a chronic, slowly progressive renal disease that will cause symptoms in children and in young adults, but that will cause chronic renal impairment in a proportion of these patients in older age. As the mean age of patients admitted into renal replacement programmes - be it peritoneal dialysis, haemodialysis or renal transplantation increases, the number of patients who need to be treated for ESRD caused by glomerular disease will remain substantial. Since the cost of renal replacement therapies are considerable - both in terms of medical and social costs - it is worthwhile to converge on tertiary prevention measures, aimed at postponing or altogether preventing the start of renal replacement therapy. Biesides attention to prevention of the complications of existing renal disease, a great deal of energy has been devoted to elucidate the pathogenetic mechanisms of renal diseases, but truly successful therapeutic interventions for idiopathic glomerulonephritis have not yet emerged from this.

For adequate tertiary prevention, knowledge of the natural history of renal disease is mandatory. The natural history of idiopathic glomerular disease, however, remains topic of debate for several reasons. The diagnosis of idiopathic glomenular disease can only be made by performing a renal biopsy, which is an invasive 
procedure but, in experienced hands, has no mortality and little morbidity. Due to the indolent course of many idiopathic glomerular diseases study of the natural history is only possible if long-term follow-up is attainable. Financing of long-term studies in the Netherlands, as in the United States, is usually limited to four years. Many reports on natural history of disease are derived from academic centres that tend to suffer from the "centre" effect: concentration of patients with more severe or more complicated disease in these centres. In this thesis we address the natural history of the most common idiopathic glomenulopathies in adults, who either present with chronic haematuria or with nephrotic syndrome: IgA nephropathy and thin glomerular basement membrane (GBM) nephropathy on the one hand and membranous glomerulonephritis (MGN) on the other hand (table 3). Both membranous glomerulonephritis and IgA nephropathy are immune-mediated and both show electron-dense immune-complexes, although in different anatomical locations, on ultrastructural analysis.

Table 1. Causes of renal failure in patients with end-stage renal-disease entering renal replacement programmes in Europe (EDTA Registry) in the period 1982-19911

\begin{tabular}{lll} 
& $1982(\%)$ & $1991(\%)^{2}$ \\
\hline Diabetes & 8 & 15 \\
Renovascular disease & 9 & 12 \\
Glomerulonephritis & 27 & 22 \\
Cystic nephropathy & 9 & 8 \\
Other hereditary nephropathy & 2 & 4 \\
Pyelonephritis & 20 & 13 \\
Drug nephropathy & 3 & 3 \\
Systemic disease & 4 & 4 \\
Others & 18 & 21
\end{tabular}

Legend to the table

1 Adapted from Mallick NP, Jones E, Selwood N. The European (European Dialysis and Transplantation Association-Etropean Renall Association) Registry. Am J Kid Dis, 1995, 25, pp 176-187

230,060 new patients in 1992 required renal replacement therapy.

Due to rounding up, the percentages do not always exactly represent 100 . 
Table 2. Causes of renal insufficiency in new patients requiring renal replacement therapy in the United States in 1991.1

patients / million population $(\%)^{2}$

Glomerulonephritis

Diabetes

Hypertension:

Cystic disease

Other
$20(12)$

$58(34) *$

$47(29)^{*}$

$39(23)$

Legend to the table

1 Adapted from Agodoa L, Eggers PW. Renal replacement therapy in the United States: data from the United States Renal Data System. Am J Kid Dis, 1995, 25, pp 119-133.

245,000 new patients entered renal replacement programmes in the United States in 1990 (incidence rate 196 per million population)

Note: In the USA population $80 \%$ of the new ESRD patients who become eligible for renal replacement therapy because of diabetic nephropathy are either black or native American, as is the case for hypertensive nephropathy.

When addressing the natural history of glomerular disease, three questions are essential:

1. Is the sign or symptom of disease with which the patient presents indicative of glomerular disease?

2. Which type of glomerular disease causes the signs and symptoms?

3. At which point in time in the natural history of glomerular disease is the patient identified?

We will illustrate these questions using the chronic haematurias and nephrotic syndrome as signs of idiopathic glomerular disease. The design of the prospective epidemiological study on idiopathic glomerular disease as carried out by the Regional Working Pary in Limburg allows these questions to be addressed. In this study renal biopsies were taken in the regional hospitals from all patients who presented with chronic haematuria of apparently non-urological origin and from all patients who presented with nephrotic syndrome of apparently recent onset. Indications for renall biopsy were in hierarchical order: recent onset uraemia (senm creatinine concentration $>300$ umol/L), and recent onset nephrotic syndrome (excretion of serum proteins into 
the urine $>5 \mathrm{~g} / \mathrm{day}$ ), the renal biopsies were taken within 6 months after patient identification. Other indications for renal biopsy were idiopathic persistent erythrocyturia or recurrent macroscopic haematuria without or with proteinuria $(0.5-5$ $\mathrm{g} / \mathrm{day}$ ), of at least six months duration, or persisting proteinuria (>1.0 g/day) of at least six months duration, in the absence of hypertension. Renal biopsies were taken within 2 years of patient identification [4].

Table 3. Epidemiology of idiopathic glomerulonephritis in the Netherlands ${ }^{1}$

\begin{tabular}{llllllll} 
Diagnosis & no. & Incidence & Indication for renal biopsy \\
biop- & per $10^{6}$ & H & HP & P & NS U \\
& sies & per vear & & & & \\
\hline
\end{tabular}

- Idiopathic

IgA nephropathy

20

$15 \quad 13^{*}$

- Thin GBM

nephropathy

127

- Membranous

nephropathy

- Focal segmental

sclerosis and

hyalinosis

- Minimal change

nephropathy

9

6

9

Legend to the table

Adapted with slight modification from Tiebosch et al. Epidemiology of idiopathic glomerulonephritis: a prospective study. Kidney International, 1987, 32, pp 112-116. $\mathrm{H}$ : recurrent macroscopic haematuria or persistent erythrocyturia, HP: haematuria with proteinuria, P: chronic proteinuria, NS: nephrotic syndrome; U: uraemia (serum creatinine $>300 \mu \mathrm{mol} / \mathrm{l}$ )

* One patient had a slightly elevated serum creatinine concentration at renal biopsy (> 120 umol/). 


\section{The chronic haematurias}

We consider, arbitrarily, haematuria to be chronic when it persists for at least 6 months. When batematuria is associated with non-orthostatic proteinuria of over 1.0 g/day, it is usually of glomerular origin. The presence of erythrocyte casts is confirmatory evidence. When an urological cause for non-proteinuric $(<0.5 \mathrm{~g} / \mathrm{day})$ haematuria has been ruled out by cystoscopy, intravenous pyelography, ultra-sound examination, CT scans or angiography and metabolic studies - e.g. measuring 24 hour urinary excretion of uric acid and calcium - the haematuria is considered to be idiopathic. The next step in diagnosing the cause of haematuria is a renal biopsy. However, this is topic of debate [5] for at least two reasons. Non-proteinuric glomerular haematuria, regardless of cause, has a benign prognosis with regard to ultimate renal function, thus renal biopsies are an academic exercise and prone to cause complications. Thus, chronic glomerular haematuria should be followed, renal biopsies should only be taken on indications like deterioration of renal function. However, until a glomerular cause for haematuria is nuled out with renal biopsy, the patient should be followed at the urological outpatient clinic for repeated, often invasive, urological work-ups.

The question pro- or contra- renal biopsy has been discussed by Topham et al [6]. They question the practice of having patients younger than 45 years of age, with chronic non-proteinuric haematuria screened first by a urologist, by pointing out that of these patients only $2 \%$ will turn out to have a urological disorder [7], which is seldom malignant [8]. In their series $50 \%$ of the patients were shown to have a glomerular or renal disorder by renal biopsy, thus they advocate to start the work-up of non-proteinuric haematuria with a renal biopsy [6]. Additional arguments in favour of such a policy included determination of prognosis, employment and insurance policies and, above all, unnecessary repeat urological follow-ups [9]. We support this policy because, as shown further in this thesis, both thin GBM nephropathy and nonproteinuric IgA nephropathy have a high incidence of late onset hypertension and occasionally renal insufficiency [10].Thus, the positive identification of these minor glomerular disorders is of prognostic significance.

It is often suggested that urinary red cell morphology can differentiate between glomerular haematuria and haematuria of urological origin. The differentiation between glomerular and urological haematuria on the basis of urinary red cell morphology was first described by Fairly and Birch [11] using semi-quantitative urinary red cell counts 
and phase contrast microscopy. Excretion of large numbers of normal, eumorphic, erythrocytes into the urine is indicative of urological disorders; excretion of dysmorphic erythrocytes, which differ from the nomal erythrocyte in terms of size, shape and haemoglobin content, indicate glomerular bleeds. [11]. Normal healthy men excrete dysmorphic erythrocytes into the urine up to $8000 / \mathrm{ml}$ in freshly woided urinary morning specimens [9]. Patients with "minor glomenular disorders", like nonproteinuric IgA nephropathy or thin GBM nephropathy, also excrete dysmorphic erythrocytes but only slightly more than normal men. Consequently, urinary red cell analysis does not differentiate nomal from minor glomerular disorders [12] However, when large numbers of erythrocytes are excreted into the urine, e.g. $100.000 / \mathrm{ml}$ in freshly woided morning urine, as may be the case in mesangiocapillary glomerulonephritis [11] or when overnight 12 hour Addis counts shows the erythrocyturia to be over 30 times of normal, as may be the case in urolithiasis [13], the red cel $\rfloor$ morphology will give clear answers, as large numbers of eumorphic erythrocytes will be present in the latter, but not in the former condition. In chronic mild microscopic haematuria, normal renal histology can not be differentiated from minor glomerular disorders by phase-contrast microscopy, while non glomerular microscopic haematuria may yield a mixed pattem. Hence, renal biopsies are required to differentiate between normal, minor glomerular disease or urological disorders.

The second question is when renal biopsies should be taken in chronic idiopathic haematuria. This question was addressed in a prospective study by taking renal biopsies from 49 patients with chronic idiopathic non-proteinuric haematuria of at least six months duration, but within 2 years of patient identification [10]. Of these 49 patients, 20 had normal renal tissue: $65 \%$ lost haematuria within 5 years. In 7 haematuria persisted which was associated with wrolithiasis in 5 , in the absence of hypercalciuria or increased excretion of uric acid identification [10]. On the basis of these observations one can defend the argument that in patients with chronic microscopic haematuria one should proceed with renal biopsy after 4 years. By doing this, $65 \%$ of the patients with normal renal tissue would not have been subjected to renal biopsy, at the expense of the patients with glomernlar haematuria who will have been subjected to repeated urological-ups. We now consider that patients with idiopathic chronic non-proteinuric erythrocyturia, younger than 40 years of age, should firstly be subjected to renal biopsy within four years of patient identification. Those patients older than 40 years of age should be firstly subjected to urological screening. 


\section{Immune-mediated versus non-immune mediated renal damage}

In IgA nephropathy immune complexes are formed in or on the mesangial cell, with some degree of subendothelial immune complex formation, leading invariably to erythrocyturia and, in most cases, to protemuria. The observation that IgA nephropathy frequently recurs in renal transplants suggests an autoantigen, while the observation that membranous glomerulonephritis almost never recurs in transplants suggests that membranous glomerulonephrits is due to trapped antigens from divers origin; either microbial, viral or neoplastic. We show (vide infra and chapters 2,3 , and 4) that these immune mediated glomerulopathies, as well as the non-immune mediated thin GBM nephropathy, may in time cause renal failure and that a final common, non-immunological, pathway exists. This is characterised histologically by glomerular scarring and nephron obliteration, focal and segmental glomerulosclerosis, occasional interstitial nephritis - probably a result of a cellular immune reaction against leakage of tubular proteins into the renal interstitium - and the effects of hypertension on renal macro- and microvasculature. This final common pathway can obviously not be reversed by immunosuppressive therapy, but may be influenced to some extent by therapies aiming at decreasing glomerular and systemic hypertension, reducing hyperfiltration by decreasing protein load and preventing secondary hyperparathyroidism $[14,15]$.

The dilemmas for the physician caring for patients with IgA nephropathy are clear: at which point in the disease history of the individual patient is hypertension, increased proteinuria or decline in renal function due to an active immunological process? When is this due to non-immunological mechanisms and, above all, can the imimune processes be halted before the non-immune mechanisms come into play? The amswers to these questions require a thorough understanding of the pathogenesis and the natural history of human glomerular disease. Animal models of glomerular diseases are instrumental in studying the pathogenesis of lesions, they have elucidated effector mechanisms of several glomerular disorders, like anti-glomerular-basementmembrane nephropathy and membranous glomerulonephritis, thus allowing manipulation of these effector" mechanisms. A clinically relevant animal model is lacking for IgA nephropathy.

Of the few animal models which allow analysis of the interaction of the immune response with an intravascularly located glomerular antigen, and the nonimmunological mechanisms leading to renal insufficiency are rat models of chronic renal allograft rejection. These chronic rejection models are relevant to IgA 
nephropathy because they illustrate the non-immunological mechanisms that come into play when a chronic renal immune injury, be it against intravascularly located antigens or against mesangially located antigens, causes sufficient loss of renal mass. This interplay of immune and non-immune factors is exemplary illustrated in the study of Tullius et al [16] where renal allografts were removed from the recipients and transplanted back into the donor strain at variable points in time. In this study extensive cellular infiltrate, mostly consisting of $\mathrm{T}$-cells and $\mathrm{ED}{ }^{+}$macrophages, combined with massive amounts of cytokines expressed in the interstitium, and glomerular and arteriolar deposition of IgM, IgG and C3 were present in allografts at 8 weeks after transplantation. This was associated with early interstitial damage, fibrosis and arteriolar inflammation. The lesions were reversible when allografts were removed from the recipients before 12 weeks after the transplantation; retransplantation of the allografts into the donor strain after 12 weeks lead to similar humoral and cellular and structural charges in the retransplanted kidney as in the nonretransplanted allograft. Thus, it was shown that in the first weeks chronic renal allograft rejection is a reversible alloantigen-driven immune response, while at the course of time adaptive mechanisms, independent of the bost environment, are responsible for progressive renal damage. In the same paper it is shown that transplantation procedure related damage and hyperfiltration also play a role in chronic rejection as isografts - which were transplanted into syngeneic animals that had undergone bilateral nephrectomies - did develop tubular atrophy, vascular obliteration with intimal thickening and arterial smooth muscle proliferation.

Many of the histological features that are seen in chronic renal allograft rejection resemble those found in other chronic glomerular diseases. Debate exist as to whether renal. histopathological findings are relevant for the prognosis of renal disease. In many reports on idiopathic glomerulonephritis histological findings are identified that appear associated with a poor prognosis. Indeed, in a study of Williams et al. [17] it was shown that renal pathological findings are important in determining the prognosis as patients with renal failure due to glomerulonephritis or diabetic nephropathy showed faster deterioration of their renal function, while patients with similar renal failure on the basis of chronic pyelonephritis or hypertensive nephropathy showed stable renal function. Histological criteria were also used in a study on chronic allograft rejection by Isonierni et al. [18]. Here, a "chronic allograft damage index" (CADD) was formulated which scored the presence of interstitial Iymphocytic inflammation and fibrosis, glomerular sclerosis and mesangiall matrix increase, vascular intimal proliferation and tubular atrophy. These lesions were 
correlated with chronic renal allograft rejection when biopsies were takien 2 years after renal transplantation. Using the $\mathrm{CADI}$, the authors were able to accurately predict the development of progressive renal failure in chronic renal allograft rejection [18]. Those patients who had a low index and stable graft function at 2 years after transplantation, showed stable renal function at 6 years follow-up, while those patients with a high index showed either progressive renal function or were dialysisdependent at follow-up. An intermediate group was identified that had a low index, but deteriorating renal function at 2 years; of these patients half showed progressive graft function loss or was on dialysis at 6 years follow-up.

Clinically, chronic renal allograft rejection is characterised by a slow, unrelenting deterioration of graft function leading in the course of several months or years to end-stage renal failure. Increased proteimuria [19] and hypertension [20] are hallmarks of chronic rejection. Identification of hypertension as a factor that directly precedes an increase in the rate of decline in renal function [20] and reports that the rate of deterioration of renal function can positively be influenced by institution of a low-protein diet in patients with chronic allograft rejection [21] support the hypothesis of a final common non-immunological pathway in which haemodynamic mechanisms are initiated to compensate for loss of functional renal tissue, but which appear deleterious for the remaining viable nephrons [22].

An interesting observation that emerged from studies in animal models of real disease and humans (in passive Heymann nephritis [23], in IgA nephropathy in patients who are treated because of active crescentic lesions [24] and in human renal allograft [25]) is that when the activity of the immune attack is high, more chronic lesions are seen in renal biopsies taken later in the course of the disease. Probably, this is related to the potentiality of active immunological lesions to initiate repair mechanisms through increased cytokine-release (mainly TGF-B) from infiltrating and glomerular cells, leading to increased extracellular matrix production and secondary mesangial and glomerular sclerosis $[26,27]$. The activity of an immune mediated renal disease may be measured on renal biopsy and used as an indication for immumosuppressive treatment. Ideally, early instituted treatment should prevent chronic lesions, while immunosuppression started later in the course of the disease risks to be less effective in terms of long-term preserwation of renal function. In chapter 4 we illustrate that the use of activity and chronicity indices in IgA nephropathy allows for accurate prediction of renal prognosis in IgA nephropathy and car provide useful guidelines in treatment of $\operatorname{Ig} A$ nephropathy 


\section{The nephrotic syndrome}

We consider a nephrotic syndrome to be present when the excretion of serum proteins into the urine equals or exceeds 5.0 gram per day, in the absence of Bence Jones proteinuria. A great variety of glomerular diseases may, at any given point in the natural history of disease, cause this degree of proteinuria. This may be of a transient nature, e.g. in diffuse extracapillary glomerulonephritis when a progressively decreasing glomerular surface area, clinically evident by an increasing serum creatinine concentration, reduces leakage of serum proteins into Bowman's space. Proteimuria may be stable; this stability is an operational definition: in the context of this thesis a period of six months is used, provided the serum creatinine concentration remains < $300 \mu \mathrm{mol} / \mathrm{L}$. These stable nephrotic syndromes are due to interference with or damage to the glomerular capillary wall by, usually, immunologically mediated disease. The nephrotic syndrome, depending on its severity, may cause divers systemic effects. Salt and water retention may be associated with generalised oedema, loss of circulating anticoagulant proteins into the urine may cause venous thrombosis, particularly at the site of maximum depletion: the renal veins [28], and hypercholesterolaemia may predispose to cardiovascular disease [29]. In severe and aselective proteinuria, the ensuing hypogammaglobulinaemia enhances bacterial infections. When an idiopathic nephrotic syndrome presents in adults with normal renal function, it is usually caused by membranous glomerulonephritis, focal and segmental glomeruiosclerosis, or minimal lesion glomerulopathy in this order of decreasing incidence $\llbracket 30]$. Since all three diseases are immune-mediated, the question arises whether to proceed with immunosuppressive therapy such as steroids, alkylating agents and/or cyclosporine immediately or to proceed with renal biopsy first.

It has been advocated, on the basis of decision analysis, to treat these patients blindly with a trial of steroid therapy before resorting to renal biopsy [31]. Although this approach is taken by paediatric nephrologists - since steroid responsive minimal lesion glomerulopathy is by far the most common cause of childhood nephrotic syndrome - this does not hold for adults [30]. Here, membranous glomerulonephritis is the most common cause of nephrotic syndrome and steroids do not influence this disease $[32,33]$. In addition, in adults with focal and segmental glomerulosclerosis a good response to steroids is less frequently observed compared to those with minimal lesion glomerulopathy [34]. Hence, in contrast to childhood nephrotic syndrome, a diagnostic therapeutic trial with steroids in adults is contraindicated. Since minimal lesion glomerulopathy is responsive to cyclosporine and since a beneficial effect of 
cyclosporine has been observed in a controlled trial in membranous glomerulonephritis [35], it may appear reasonable to use cyclosporine therapy as first step procedure in nephrotic syndrome in adults before resorting to renal biopsy. This approach cannot be recommended for three reasons. Firstly, most patients with membranous glomerulonephritis go into spontaneous remission. Secondly, (vide infra) an immunologically driven membranous glomerulonephritis may, without any change in proteinuria, convert to a non-immune driven proteinuria, due to remodelling of the GBM or development of focal and segmental glomerulosclerosis. This, obviously, is not responsive to cyclosporine therapy but should be treated with $A C E$ inhibition. Finally, cyclosporine A may influence renal function adversely. Hence, in adult nephrotic syndrome one should proceed with renal biopsy first [36]. This allows a clear differentiation of membranous glomerulonephritis, focal and segmental glomerulosclerosis, minimal lesion glomenulopathy and other causes of nephrotic syndrome (table 4).

The question remains whether - in membranous glomerulonephritis - the renal biopsy gives information about prognosis. This requires insight into the pathogenesis of membranous glomerulonephritis and its natural history (see Chapter 5).

\section{Renal biopsy findings in membranous glomerulonephritis}

In idiopathic membranous glomerulonephritis several stages are identified and thought to reflect the evolution and severity of disease [37, 38]. Histological classification according to Noel et al. [39] uses silver impregnation on 1-2 $\mu \mathrm{m}$ sections, counterstained with hematoxylin and eosin. Stage 1 disease is characterised by normal histology on light microscopic examination (stage la) or focal and segmental silver positive subepithelial spikes (stage $\Perp$ b). This is invariably associated with the presence of $\operatorname{IgC}, \mathrm{C} 3$ and the membrane-attack-complex (MAC, C5b-9) in a diffuse granular capillary pattern on immunohistochemistry, while newly formed and irregular subepithelial electron dense deposits with occasional basement membranelike protrusions are visible on ultrastructural analysis. Stage 2 is characterised histologically by diffuse subepithelial silver-positive spiking with sometimes and segmental inclusion of the deposits. Immunohistochemistry is analogous to stage 1 disease. Transmission electron-microscopy reveals basement membrane-like protrusions and frequent focal and segmental incorporation of the deposits in newly formed GBM. Stage 3 is characterised by diffuse thickening of the GBM with honey- 
comb appearance histologically, since electron dense deposits are covered by newly formed basement membrane. Immunohistochemistry revealls IgG, wsually without C3 or MAC. By transmission electron-microscopy stage 3 is divided, according to Churg [40], into stage III, characterised by diffuse fusion of the GBM around the deposits, rarefaction of the deposits and thickening of the GBM, and stage IV with disappearance of the deposits leaving an irregularly thickened lamina densa or (stage V) an almost normal GBM. Mesangial proliferation is seen in a small percentage of the biopsies. Progression can be accompanied by the development of focal and segmental glomerulosclerosis (FSGS), probably related to hyperfiltration in the glomeruli [41], and with capillary collapse, capsular adhesions and glomerular sclerosis [40].

Table 4. Renal biopsies taken because of nephrotic syndrome ${ }^{\#}$ of unknown origin. Prospective study of the Regional Working Party on Renal Disease in Limburg

$1978-1987^{\mathbb{I I}}$

Membranous nephropathy

- jidiopathic

24

- secondary

3

Minimal change nephropathy

14

Focal and segmental sclerosis and hyalinosis

11

Membranoproliferative glomerulonephritis

4

Divers

$21 *$

Legend to the table

Excretion of serum proteins into the urine over 5 grams per day

TAdherent adult population at risk between 18 and 65 years was $2.5 \times 10^{6}$.

Secondary membranous nephropathy occurred in the context of carcinoma, lepra, rheumatoid arthritis and hepatitis $B$ infection.

* Acute glomerulonephritis $(x 6)$, focal segmental pauci-immune extracapillary $G N$ (x5), IgA nephropathy (x3), chronic interstitial nephritis with FSGS (x2).

AL amyloidosis (x2), Immunotactoid $\mathrm{GN}(\mathrm{x} 1)$, diffuse mesangioproliferative $\mathrm{GN}(\mathrm{x} 2)$. 
The temporal sequence in sequence in membranous nephropathy has also been illustrated in immunohistochemical studies where in stage I membranous nephropathy no new collagen formation is detectable, while in subsequent stages novel type IV ( $\alpha 3-0.4)$ collagen chains, together with laminin and nidogen, is deposited in close apposition to the traditional type IV $(\alpha 1-\alpha 2)$ collagen chains [42]. However, repeat renal biopsies have shown that this temporal sequence does not always represent the course of membranous nephropathy in the individual patient.

\section{Pathogenesis of the lesions in membranous glomerullonephritis}

Idiopathic membranous glomerulonephritis is the most common cause of nephrotic syndrome in adults (table 4) [30]. In 10-20\% of the patients, membranous glomerulonephritis is associated with malignant neoplasm (particularly of the lung [43]), auto-immune disease like systemic lupus erythematosus, chronic infections of parasitic (e.g. malaria), bacterial (e.g. lepra), or viral (e.g. hepatitis B, Figure la, b) origun or drugs. When secondary causes have been ruled out, membranous glomerulonephritis is classified as idiopathic. Work on experimental membranous glomerulonephritis in rodents has unequivocally established that the disease is brought about by an IgG-antibody response to trapped or auto-antigens residing in the subepithelial part of the glomerular wall, thus causing local immune complex formation $[44,45]$. In man planted antigens of microbial (fig 1) or neoplastic nature have been demonstrated to reside in the immune complexes in secondary membranous glomerulonephritis. Once the immune complexes are formed, the ensuing complement activation contributes to the proteinuria [46]. A pivotal factor in the development of proteinuria is the deposition of the C5b-9 teminal complex at the podocyte [47]. The C5b-9 complement complex does not induce lysis of the glomerular epithelial cell, but stimulates the release of inflammatory mediators [48] and the production of laminin and collagen type IV $[42,49,50]$, which are subsequently deposited in the GBM where they contribute to the formation of the typical spikes. However, C5b 9 is not the sole factor responsiblle for the proteinuria in Heymann nephritis, since active Heymann nephritis with proteinuria can be induced in $\mathrm{C6}$ deficient rats [51], while depletion of CD8* T-cells mitigated proteinuria [52]. The ongoing glomerular inflammatory reaction with ensuing proteinuria may lead to sequallae like remodelling of the glomerular basement membrane, focal and segmental glomerulosclerosis, 
interstitial cellular infiltrates and frbrosis, ultimately resulting in nephron loss and renal insufficiency [53-55].

\section{Immunosuppression in iMGN: role of renal biopsy}

These lesions described above appear to make a solid case for immunosuppressive therapy (steroids and cytostatic drugs), but this remains topic of debate for at least three reasons. Firstly, the natural history of patients with early idiopathic MGN with similar degrees of proteinuria is highly variable and is characterised by spontaneous remissions and recurrences, stable nephrotic syndrome over many years and early and late renall insufficiency $[39,56]$. Secondly, follow-up of pharmacological interventions studies is usually of short duration (2-5 years), and thirdly, the incidence of idiopathic membranous nephropathy is low. This triad of factors results in studies in which large groups of patients are treated for short periods of time without taking into account the natural history of disease. Because of the low incidence of disease, these studies are usually multi-centre studies, suffering from bias as prognostically poor patients and patients late in the natural history of disease are referred to these centres. This explains why the large prospective study from Boston [57] showing a beneficial effect of high dose steroids given for 8 weeks, could not be confirmed by the Toronto study [32] nor by the Medical Research Council Trial [33]. This point is exemplary illustrated by the prospective and multi-centre Italian study carried out by Ponticelli et al [58], where controls and treated groups appeared to be well matched for duration of disease and histological staging. After a follow-up of 2.5 years the group treated with chlorambucil and prednisone fared much better compared to the controls in terms of severity of proteinuria and incidence of renal insufficiency [58]. This effect, however, was no longer seen at a follow-up of 4 years [59]. Similar arguments can be used for studies using oral or intravenously administered bolus cyclophosphamide [60-63]. It is clear from the above mentioned, that only those patients with idiopathic membranous glomerulonephritis who have an immunemediated risk factor for renal insufficiency should be treated with immunosuppressive drugs. This risk factor should preferably be apparent early in the disease history. We show in Chapter 5 that no such risk factors for renal insufficiency are identifiable early in the disease history, since almost all patients with idiopathic membranous glomerulonephritis present with stage I histology, normal blood pressure, normal renal function and a proteinuria of over $5.0 \mathrm{~g} /$ day. Similarly, the severity of initial proteinuria has no bearing on renal prognosis (chapter 5), in contrast to the presence of 
chronic severe proteinuria as is indicated in our study (chapter 5) as well as in the study from the Toronto Registry. In the Toronto Registry study, chronic proteinuria over $6 \mathrm{~g} / \mathrm{day}$ present for over 9 months is a risk factor for renal insufficiency (bigh predictive value), while the degree of proteinuria, gender, age and blood pressure were not [64]. When chromic proteinuria is associated with decreasing renal function, the positive predictive value for renal insufficiency increases [65]. Precisely these patients are treated with - and frequently responsiwe to - immunosuppressive medication, e.g. oral cyclophosphamide [66]. These observations raise the question whether all patients with chronic sustained proteinuria, e.g. of over 2 years duration, should be treated with immunosuppressive drugs or only those with decreasing renal function. We believe that persistent proteinuria in combination with decreasing renal function should be treated immunosuppressively, provided repeat renal biopsies are taken to differentiate immune-mediated proteinuria from proteinuria due to aspecific mechanisms, as illustrated by the case history presented below.

A 20 year old male with a chronic hepatitis $B$ infection - carrier state - with normal liver function tests, HbsAg and Hbe-antigens positive, antibodies to Hbcore antigen positive (in serum) developed an acute nephrotic syndrome diue to stage 1 (fig. la) membranous glomerulonephritis. The subepithelial immune complexes contained IgG, C3 and MAC as demonstrated by direct immunofluorescence and Hbe-antigen by immunoperoxidase staining (fig. 1b). Transmission electronmicroscopy revealed the presence of subepithelial electron dense deposits compatible with stage I membranous glomenulonephritis. Because of severe proteinuria, 15-20 g/day, the patient was treated 8 months after onset of mephrotic syndrome with Vidarabine $5 \mathrm{mg} / \mathrm{kg} /$ day intramuscularly for 28 days after which the converted within 8 weeks to $\mathrm{Hbs} \mathrm{Ag}$ - and Hbe-antibody positivity. As the nephrotic syndrome continued relentlessly, a repeat renal biopsy was taken 4 months after seroconversion -14 months after onset of discase. This repeat renal biopsy showed histologically, conspicuous stage II and focal stage III lesions of membranous glomerulonephritis with strong staining of the immuneaggregates for $\mathrm{IgG}$ and $\mathrm{C} 3$. The staining for Hbe had clearly decreased (fig. $1 \mathrm{c}_{\mathrm{n}} \mathrm{d}$ ). Transmission electron-microscopy showed already ongoing remodelling of the GBM. Because of persisting, although decreasing, proteinuria (of 5 g/day) a third renal biopsy was taken 30 months after onset of disease. Now the glomerular capillary wall was completely remodelled histologically, which was confirmed by transmission electron-microscopy, which demonstrated absence of electron dense deposits. This was associated immunohistochemically with the absence of $\mathrm{C} 3$ and Hbe-antigen from the glomerulli which stained only for IgG in an aspecific patten (fig. 1e, f). Since the patient had a stable - and normal serum creatinine concentration throughout the disease history, it is clear that in the first repeat biopsy the nephrotic syndrome was shown to be driven by immune reactants up to 4 months after 
seroconversion. After this time the nephrotic syndrome was caused by aspecific mechanisms such as remodelling of the GBM.

Legend to figure 1.

A. Membranous nephropathy stage 1 , related to active Hepatitis B infection, silver methenamin hematoxylin eosin stain. In this biopsy slight swelling of the visceral epithelial cells is visible, otherwise the glomerulus shows no abnormalities. The patient presented with a recent onset nephrotic syndrome and liver test abnomalities, he was hepatitis $B$ antigen positive and antibody negative $(160 x)$. $B$. Immunohistochemistry of the same biopsy shows diffuse capillar granular deposition of the Hepatitis B core antigen (immunoperoxidase staining) (160x). C. Repeat renal biopsy, taken because of persistent nephrotic syndrome and decrease in renall function. six months after the initial biopsy, revealed a stage II-IV membranous nephropathy with extensive interstitial mononuclear cell infiltrate, responsible for the renal failure. The patient had received anti-viral treatment, leading to seroconversion and disappearance of the hepatitis $\mathrm{B}$ antigen $(65 \mathrm{x})$. D. Immunohistochemistry of the first repeat renal biopsy showed only residual presence of the Hepatitis B core antigen in the glomerulus (immunoperoxidase) $(160 \mathrm{x}$ ). E. Three years after initial biopsy the patient still had persistent nephrotic syndrome. The second repeat renal biopsy revealed focal and segmental hyalinosis and sclerosis with lesion at the glomerular tip and the vessel pole (arrows) (160x). F. Immunofluorescence showed positive staining for $\mathrm{IgG}$, in a capillar linear pattern. $\mathrm{C} 3$ was negative and $\mathrm{Clq}$ was positive in an identical pattern as for $\operatorname{IgG}(160 \mathrm{x})$. 
Figure 1.
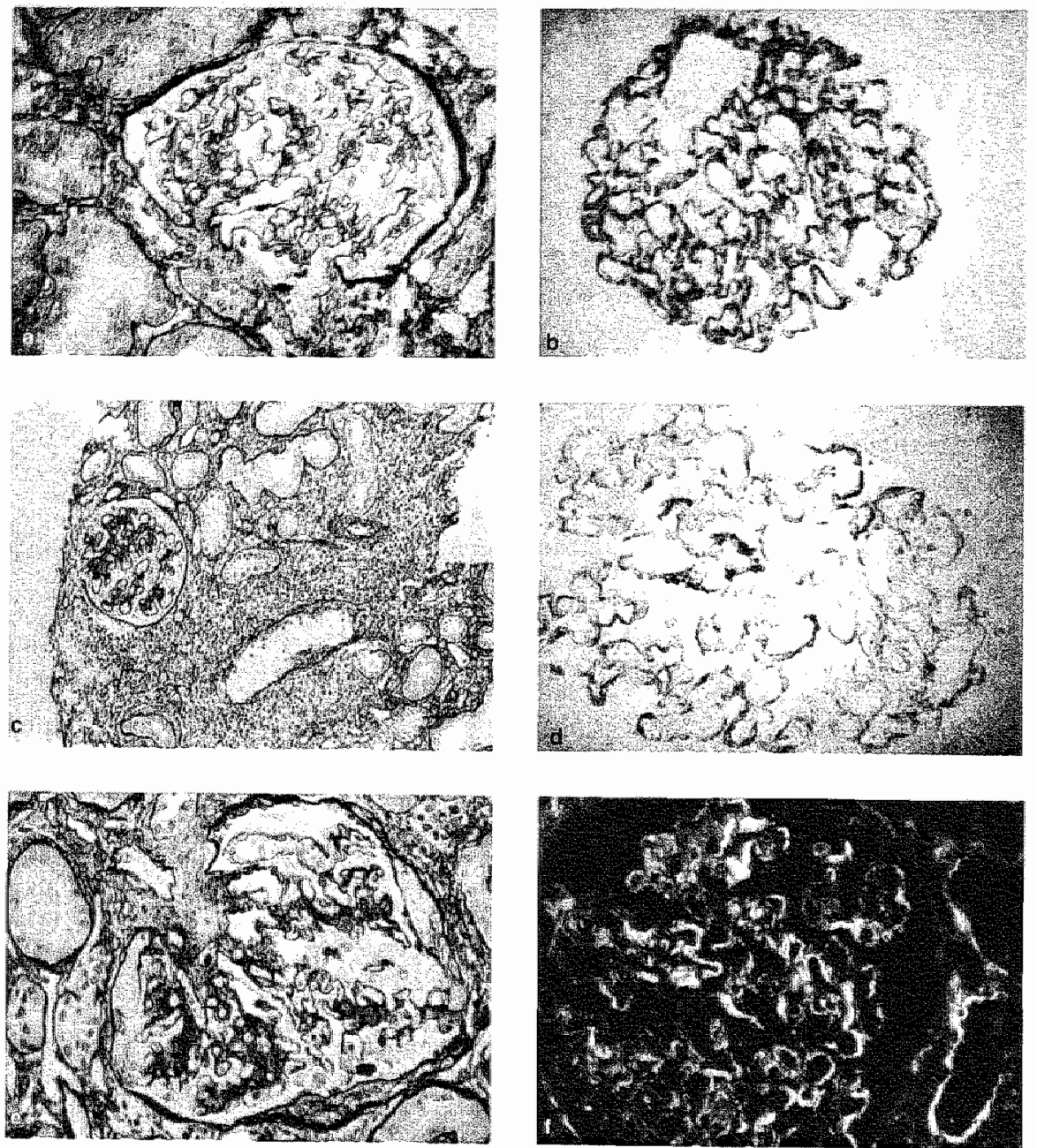
Lately it has been suggested that one might do without repeat biopsy in order to determine whether or not $\mathrm{IMGN}$ is immunologically active by determining C5b-9 neoantigen in the urine. This approach derives from work on the rat model of passive Heymann nephritis, where it was shown by ex viwo perfusion of kidneys that $\mathrm{C} 8$ (and hence MAC) was required for visceral epithelial cell damage and proteinuria [67]. In the same model of passive Heymann nephritis it was shown in vivo that the MAC is first inserted in the cell membrane of the epithelial cell, then selectively endocytosed and transported across the epithelial cell in order to be exocytosed into the urinary space [68]. It was also shown that in passive Heymann nephritis the urine contained. the MAC [69]. This issue was taken up clinically where the question was asked whether enhanced MAC excretion into the urine was specific for immunologically active membranous glomerulonephritis. In order to answer this question MAC was determined in the urine of patients with nephrotic syndrome of divers causes; greater amounts of MAC were found in the urine of patients with focal and segmental glomerulosclerosis and diabetic nephropathy, but not in membranous glomerulonephritis [70]. These authors showed by immunohistochemical studies of renal biopsies the MAC complex to be of tubular origin. The issue was taken up by Schulze et al [71] who measured the excretion of the MAC relative to $C 5$, in order to correct for C5 dependent postglomerular, tubular MAC generation. Using this correction, they observed increased excretion of $\mathrm{MAC}$ relative to $\mathrm{C5}$ in immune complex mediated glomerular diseases of divers origin when compared to e.g. minimal lesion nephropathy. Interestingly, of the 40 patients with membranous glomerulonephritis only 9 had increased excretion of MAC into the urine. This study does not answer the question whether in membranous glomerulonephritis enhanced. excretion of MAC over $\mathrm{C5}$ is indicative of active immune mediated gllomerular disease, as no renal biopsies were taken. It is clear that the measurement of C5b-9 neoantigen, even when corrected for excretion of $\mathrm{C5}$, does not answer the question whether or not at any given point in time the proteinuria in $\mathrm{iMGN}$ is immune driven or is due to aspecific mechanisms. This requires a renail biopsy.

\section{Summary}

In this Chapter we have reviewed the role of renal biopsy in charting disease history in primary IgA nephropathy and membranous glomerulonephritis. Both are immune-mediated glomerular diseases, that present either with chronic haematuria or with nephrotic syndrome. It is pointed out that in chronic non-proteinuric mild 
haematuria, renal biopsies are required to differentiate glomerular from urological causes; and that in adults younger than 40 years of age a renal biopsy is the diagnostic procedure of choice. Provided renal biopsies are taken early in the natural history of IgA nephropathy, measuring activity and chronicity indices is of prognostic significance in terms of predicting late renal insufficiency. This is probably caused by the severity of early onset immunological injury which dictates to a considerable extent late onset, non-immune, scarring.

Review of the data of idiopathic membranous glomerulonephritis reveals that many therapeutic trials have been conducted without taking into account the natural history of disease, which includes early and late remission and recurrences, stable nephrotic syndrome and renal insufficiency. So far, the only risk factor for renal insufficiency is chronic sustained proteinuria, particularly when associated with decreasing renal function, but this conclusion was not arrived at in a prospective study taking into account the natural history of disease. We estimate that in cases of chronic sustained proteinuria, repeat renal biopsies are required to separate immune-mediated proteinuria, susceptible to immunosuppressive therapy, from the proteinuria due to aspecific mechanisms. 


\section{References}

1. Ooijendijk W., Bloemhof A., Chorus A., Miedema H. Investeren in de preventie van nierziekten een investering in de toekomst. TNO publicatie 1994

2. Mallick N., Jones E., Selwood N. The European (European Dialysis and Transplantation Association-European Renal Association) Registry. Am J Kid Dis 1995; 25: 176-187.

3. Agodoa L., Eggers P. Renal replacement therapy in the United States: data from the United States Renal Data System. Am J Kid Dis 1995; 25: 119-133.

4. Tiebosch T. Idiopathic glomerular disease, a prospective regional study. Maastricht 1987.

5. Editorial. Thin membrane nephropathy - how thin is thin. Lancet 1990; 336: 469470.

6. Topham P., Harper S., Furness P., Harris K., Walls J., Feehally J. Glomerular disease as a cause of isolated microscopic haematuria. Q J Med 1994; 87: 329 . 335 .

7. Carson C., Segura J., Greene L. Clinical importance of microhematuria. JAMA $1979 ; 241: 149-150$.

8. Froom P., Ribak K., Benbassat J. Significance of microhematuria in young adults. BMJ 1984; $288: 21-21$.

9. Birch D., Fairley K. Hematuria: glomerular or non-glomerular? Lancer 1979; 845846.

10. Nieuwhof C, Doorenbos C., Grave W., De Heer F., De leeuw P., Zeppenfeldt E, Van Breda Vriesman P. A prospective study of the natural history of idiopathic non-proteinuric hematuria. Kidney Int $1996 ; 49: 222-225$.

11. Fairly K., Birch D. Hematuria: a simple method for identifying glomerular bleeding. Kidney Int 1982; 21: 105-108.

12. Köhler H., Wandel E., Brunck B. Acanthocyturia - A characteristic marker for glomerular bleeding. Kidney Int 1991; 40: 115-120.

13. Andres A., Praga M., Bello I. , Diaz-Colon I. , Gutierrez-Millet V., Morales J. , Rodicio J. Haematuria due to hypercalciuria and hyperuricosuria in adult patients. Kidney Int 1989; 36: 9699.

14. KJahr S., Levey A., Beck G., Caggiula A., Hunsicker L., Kusek J., Striker G. , for the Modification of Diet in Renal Disease Study Group. The effects of dietary protein restriction and blood-pressure control on the progression of chromic renal disease. N Engl J Med 1994; 330: 877-884. 
15. Narins R, Cortes $P$. The role of dietary protein restriction in progressive azotemiá. Editorial. N Engl J Med 1994; 330: 929-930.

16. Tullius S., Hancock W., Heemann U., Azuma H., Tiney N. Reversibility of chronic renal allograft rejection. Transplantation 1994; 58: 93-99.

17. Williams P., Fass G., Bone J. Renal pathology and proteinuria determine progression in untreated mild/moderate chronic renal failure. Q J Med 1988; New Series 67: $343-354$.

18. Isoniemi H., Taskinen E., Hayry P. Histological chronic allograft damage index accurately predicts chronic renal allograft rejection. Transplantation 1994; 58: 1195-1198.

19. Diamond J., Tilney N., Frye J., Dinco G., McElroy J., Pesek-Diamond I., Yano H. Progressive albuminuria and glomerulosclerosis in a rat model of chronic renal. allograft rejecion. Transplantation 1992; 54: 710-716.

20. Modena F., Hostetter T., Salahudeen A., Najarian J., Matas A., Rosenberg M. Progression of kidney disease in chronic renal transplant rejection. Transplantation $1991 ; 52: 239-244$.

21. Feehally J., Bennett S., Harris K., Walls J. Is chronic renal transplant rejection a non-immunological phenomenon? Lancet 1986; 486-488.

22. Brenner B., Meyerm T., Hostetter T. Dietary protein intake and the progressive nature of kidney disease: the role of hemodynamically mediated glomerular injury in the pathogenesis of progressive glomerular sclerosis in aging, renal ablation and intrinsic renal disease. N Engl J Med 1982; 307: 652-659.

23. Couser W., Schulze M., Pruchno C. Role of C5b-9 in experimental membranous nephropathy. Nephrol Dial Transplant 1992; Suppl.1: 25-31.

24. Rostoker G. Desvaux-Belghiti D., Pilatte Y., Petit-Phar M., Philippon C.. Deforges L., Terzidis H., Intrator I., André C., Lagrue G., Lang P., Weil B. High-dose immunoglobulin therapy for severe IgA nephropathy and Henoch Schönlein purpura. Ann Int Med 1994; 120: 476-484.

25. van Saase J., van der Woude F., Thorogood I., Hollander A., van Es L., Weening J., van Bockel J., Bruijn A. The relation between acute vascular and interstitial renal allograft rejection and subsequent chronic rejection. Transplantation 1995 ; $59: 1280-1285$.

26. Schlöndorff D. Roles of the mesangium in glomenular function. Kidney Int 1996; 49: $1583-1585$.

27. Roy-Chaudhury P., Wu B., King G., Carnpbell M., Macleod A., Haites N., Simpson J., Power D. Adhesion molecule interactions in human 
glomerulonephritis: importance of the tubulointerstitium. Kidney Int 1996; 49: 127-134.

28. Llach F. Hypercoagulability, renal vein thrombosis and other complications of the nephrotic syndrome. Kidney Int 1985; 28: 429-439.

29. Kaysen G. Hyperlipidemia of the nephrotic syndrome. Kidney Int 1991; 39: 815.

30. Tiebosch A., Wolrers J., Frederik P., van de Wiel T., Zeppenfeldt E., van Breda Vriesman P. Epidemiology of idiopathic glomerular disease: A prospective study. Kidney Int 1987; 32: 112-116.

31. Kassirer J. Is renal biopsy necessary for optimal management of the idiopathic nephrotic syndrome? Kidney Int 1983; 24: 561-575.

32. Cattran D., Delmore T., Roscoe J., Cole E, Cardella C., Charron R., Ritchie S., and the Toronto Glomerulonephritis study group. A randomized controlled trial of prednisone in patients with idiopathic membranous nephropathy. N Engl J Med 1989; 320: 210-215.

33. Cameron J.S., Healy M., Adu D. The Medical Research Council Trial of shortterm high-dose alternate day prednisolone in idiopathic membranous nephropathy with nephrotic syndrome in adults. Q J Med 1990; New Series 74: 133-156.

34. Ponticelli $C$. Focal and segmental glomerulosclerosis: to treat or not to treat. Nephrol Dial Transplant 1995; 10: 2351-2354.

35. Ambavalan S., Fauvel J., Sibley R., Myers B. Mechanism of the antiproteinuric effect of cyclosporine in membranous glomerulonephritis. J Am Soc Nephro] 1996; 7: 290-298.

36. Orth S., Ritz E. Medical Progress: The nephrotic syndrome. N Engl J Med 1998; 338: 1202-1211.

37. Ehrenreich T., Churg J. Pathology of membranous nephropathy. Pathology Annual 1968; 3: 145-186.

38. Gluck M., Gallo G., Lowenstein J., Baldwin D. Membranous glomerulonephritis, evolution of clinical and pathological features. Ann Int Med 1973; 78: 1-12.

39. Noel L., Zanetti M., Droz D., Barbanel C. Long-term prognosis of idiopathic membranous glomerulonephritis. Am J Med 1979; 6: 82-90.

40. Churg J., Bernstein J., Glassock R.: Renal Disease (second edition). Tokyo-New York, Igaku-Shoin, 1995. 
Chapter I

41. Howie A., Lee S., Green N., Newbold K., Kizaki T., Koram A., Richards N., Micheal J., Adu D. Different clinicopathological types of segmental sclerosing glomerular lesions in adults. Nephrol Dial Transplant 1993; 8:590-599.

42. Kim Y., Butkowski R., Burke B., Kleppel M., Crosson J., Katz A., Michael A. Differential expression of basement collagen in membranous nephropathy. Am J Pathol 1991; 139: 1381-1388.

43. Richard-Mendes da Costa C., Dupont E., Hamers R., Hooghe R., Dupuis F., Potvliege R. Nephrotic syndrome in bronchogenic carcinoma: report of two cases with immunochemical studies. Clin Nephrol 1974; 2: 245-251.

44. Van Damme B., Fleuren G., Bakker W., Vernier L., Hoedemaeker Ph. Experimental glomerulonephritis in the rat induced by antibodies against tubular antigens. V Fixed glomenular antigens in the pathogenesis of heterologous immune complex glomerulonephritis. Lab Invest 1978; 38: 502-510.

45. Couser W., Steinmuller D., Stilmant M., Salant D., Lowenstein L. Experimental glomerulionephritis in the isolated perfused rat kidney. J Clin Invest 1978; 62: 1275-1287.

46. Couser W. Salant D. In situ immune complex formation and glomerular injury. Kidney Int 1980; 17; 1-13.

47. Sallant D., Darby C., Couser W. Experimental membranous glomerulonephritis in rats. J Clin Invest 1980; 66:71-81.

48. Cybulsky A., Salant D., Quigg R., Badalamenti J., Bonventre J. Complement C5b-9 complex activates phospholipases in glomerular epithelial cells. Am J Physiol 1989; 257: F826-F837.

49. Fukatsu A., Matsuo S., Killen P., Martin G., Andres G., Brentjens J. The glomerular distribution of type TV collagen and laminin in human membranous glomerulonephritis. Hum Pathol 1988; 19: 64-68.

50. Floege J., Johnson R., Gordon K., Yoshimura A., Campbell C., Iruela-Arispe L., Alpers C., Couser W. Altered glomerular extracellular matrix synthesis in experimental membranous nephropathy. Kidney Int 1992; 42: 573-585.

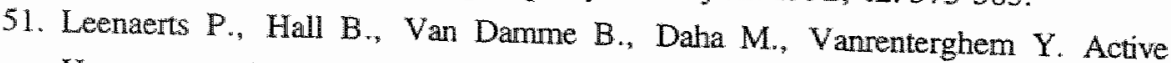
Heymann nephritis in complement component C6 deficient rats. Kidney Int 1995; 47: $1604-1614$.

52. Quiza C., Leenaerts $\mathbb{P}$., Hall B. The role of $T$ cells in the mediation of glomerular injury in Heymann nephritis in the rat. Int Immunol 1.992; 4: 423-432.

53. Cameron JS. Proteinuria and progression in human glomerular diseases. Am J Nephrol $1990 ; 10: 81-87$. 
54. Remuzzi G, Zoja C., Bertani T. Glomerulonephritis. Current Opinion in Nephrology and Hypertension 1993; 2: 465-474.

55. Lee $\mathrm{H}$., Koh $\mathrm{H}$. Nature of progressive glomerulosclerosis in human membranous nephropathy. Clin Nephrol 1993; 39; 7-16.

56. Donadio J., Torres V., Velosa J., Wagoner R., Holley K., Okamura M., Ilstrup D., Che C. Idiopathic membranous nephropathy: the natural history of untreated patients. Kidney Int 1988; 33: 708-715.

57. Collaborative study of the adult idiopathic nephrotic syndrome. A controlled study of short-term prednisone treatment in adults with membranous nephropathy. $\mathrm{N}$ Engl J Med 1979; 301: 1301-1306.

58. Ponticelli C., Zucchelli P., Imbasciati E., Cagnoli L., Pozzi C., Passerini P., Grassi C., Limido D., Pasquali S., Volpini T., Sasdelli M., Locatelli F. Controlled trial of methylprednisolone and chlorambucil in idiopathic membranous nephropathy. N Engl J Med 1984; 310: 946-950.

59. Ponticelli C., Zucchelli P. , Passerimi P., Cesana B., and the Italian Idiopathic Membranous Nephropathy Treatment Study Group. Methylprednisolone plus chlorambucil as compared with methylprednisolone alone for the treatment of idiopathic membranous nephropathy. N Engl J Med 1992; 327: 599-603.

60. Murphy B., McDonald I., Fairley K., Kincaid-Smith P. Randomized controlled trial of cyclophosphamide, warfarin and dipyidamole in idiopathic membarnous nephropathy. Clin Nephrol 1992; 37: 2229-234.

61. West M., Jindal K., Bear R., Goldstein M. A controlled trial of cyclophosphamide in patients with membranous glomerulonephritis. Kidney Int $1987 ; 32: 579-584$.

62. Falk R., Hogan S., Muller K., Jennette C., and the Glomerular Disease Collaborative Network. Treatment of progressive membranous glomerulopathy, a randomized trial comparing cyclophosphamide and corticosteroids with corticosteroids allone. Ann Int Med 1992; 116:438-445.

63. Jindal K., West M., Bear R., Goldstein M. Long-term benefit of therapy with cyclophosphamide and prednisone in patients with membranous glomerulonephritis and impaired renal function. Am J Kid Dis 1992; XIX: 61-67.

64. Pei Y., Cattran D., Greenwood CMT. Predicting chronic renal insufficiency in idiopathic membranous glomerulonephritis. Kidney Int 1992; 42:960-966.

65. Cattran D., Pei Y., Greenwood C., Ponticelli C., Passerini P.* Honkanen E. Validation of a predictive model of idiopathic membranous nephropathy: its clinical and research implications. Kidney Int 1997; 51: $901-907$. 
66. Reichert L., Huysmans F., Assmann K., Koene R. Preserving renal function in patients with membranous glomenlopathy. Ann Int Med 1994; 121: 328-333.

67. Cybulsky A., Rennke H., Feintzeig I., Salant D. Complement-induced glomentar epithelial cell injury. J Clin Invest 1986; 77: 1096-1107.

68. Kerjaschki D., Schulze M. Binder S., Kain R., Ojha P., Susani M., Horvat R., Baker $P_{.,}$Couser W. Transcellular transport and membrane insertion of the C5-9 membrane attack complex of complement by glomerular epithelial cells in experimental membranous nephropathy. J Immunol 1989; 143: 546-552.

69. Pruckno C., Bexens M., Schulze M., Johasm R. , Baker P., Couser W. Urinary excretion of $\mathrm{C} 5 \mathrm{~b}-\mathrm{C} 9$ reflect disease activity in passive Heymann nephritis. Kidney Int 1989; 36:65-71.

70. Ogrodowski J., Hebert L., Sedmak D., Cosio F., Tamerius J., Kolb W. Measurement of SC5b-9 in urine in patients with the nephrotic syndrome. Kidney Int 1991; 40: 1141-1147.

71. Schulze M., Donadio J., Pruchno C., Baker P., Johnson R., Stahl R., Watkins S., Martin D., Wurzner R., Gotze O., Couser W. Elevated urinary excretion of the C.5b-9 complex in membranous nephropathy. Kidney Int 1991; 40: 533-538. 
39 


\section{Chapter 2.}

A prospective study of the natural history of idiopathic non-proteinuric haematuria 


\section{Summary}

In a prospective study of idiopathic glomerulonephritis we determined the natural history of 49 adult patients (12 primary IgA nephropathy, 13 thin GBM nephropathy. 20 nomal renal tissue and 4 miscellaneous nephropathies) who presented with idiopathic non-proteinuric non-azotaemic haematuria of at least six months duration, in the absence of hypertension and with a negative urological work-up. Median followup was 11 years with a range of $8-14$ years.

At the end of follow-up renal function had remained stable in all subsets exept for those with miscellaneous disease. Hamaturia was still present in all patients with thin GBM nephropathy, in all but two patients with IgA nephropathy who went into immunopathological remission, in three out of four miscellaneous nephropathies and in seven out of 20 patients with nomal renal tissue. Of the latter patients five had a history suggestive of urolithiasis at follow-up, which was in the absence of hypercalciuria and hyperuricosuria. Seven thin GBM patients, five IgA nephropathy patients and three miscellaneous nephropathies developed hypertension; the incidence of hypertension in each subset was significantly higher thar in patients with normal renal tissue.

The study shows that in young adults with idiopathic chronic non-proteinuric haematuria of 4 years duration, renal biopsy will give a definite diagnosis in $86 \%$ of the patients, and that those patients with so-called minor glomerular diseases are at high risk for hypertension. Those patients with normal renal tissue have a bigh incidence of urolithiasis and should have an urological follow-up. 


\section{Introduction}

Chronic non-azotaemic and non-proteinuric haematuria, either recurrent macroscopic haematuria or persistent erythrocyuria, is usually considered, contrary to proteinuric haematuria, to be of urological origin. Patients presenting with these symptoms are referred to the urologist and are often subjected to several invasive diagnostic procedures. However, several nephrological diseases such as thinglomerular-basement-membrane nephropathy (thin GBM nephropathy), IgA nephropathy or interstitial nephritis, are known to present with isolated haematuria [1]. The prognosis of these haematuric non-proteinuric patients is considered to be benign, but precise long-term follow-up data are lacking, and defined cohorts of patients with appropriate controls are not available. In this paper we report on the long-term followup of 49 patients with chronic non-proteinuric haematuria, both of glomerular and of non-glomerular origin, who were derived from a prospective epidemiological study of idiopathic glomerulonephritis conducted between 1978-1984 in the Netherlands.

\section{Patients and Methods}

Patients were derived from a prospective epidemiological study conducted between 1978 and 1984 in three regional hospitals in the Netherlands, which served a total adherent population of 1.5 million. In this study incidence and natural history of idiopathic glomerulonephritis were determined by renal biopsy according to strict indications [1]. For a patient to be included in this arnalysis, chronic non-azotaemic haematuria had to be present for more than six months, but, to ensure evaluation of patients early in the natural history of disease, renal biopsy had to be performed within 2 years after patient identification. Urological examination, including intravenous urography, ultrasonography, cystoscopy and, occasionally, renal arteriography had to be negative. Patients with known systemic disease, liver disorders, chronic bypertension or from known families with hereditary renal disease were excluded from anallysis.

Eighty-one adult patients, aged 16 to 65 years, were biopsied because of recurrent macroscopic haematuria or persistent erythrocyturia, defined as the consistent presence of more than five erythrocytes per high power field of centrifuged urine. Of these 81 patients, five patients ( 1 idiopathic IgA nephropathy, 2 mesangiopioliferative glomerulonephritis and 2 with interstitial nephritis) presented with an increased serum 
creatinine concentration ( $>140 \mu$ mol/) and hence were excluded. Two patients with thin GBM nephropathy and one patient with focal global glomerulosclerosis died during follow-up due to pancreatic carcinoma, lung carcinoma and a malignant lymphoma. Twenty-four patients were biopsied because of chronic haematuria in combination with significant proteinuria ( $>500 \mathrm{mg} /$ day), and therefore were excluded from this analysis. Thus 49 patients, of whom 12 patients with idiopathic IgA nephropathy. 13 patients with thin GBM nephropathy, 20 patients with normal renal tissue and 4 with miscellaneous nephropathies ( 2 focal lymphocytic interstitial nephritis, 1 old interstitial nephritis and 1 focal global glomerulosclerosis) were analysed. In the cases of active interstitial nephritis the renal disorder was not caused by previous nephrotoxic medication and was not treated with steroids. None of the patients with thin GBM disease was consanguinous or had knowledge of familial hematuria at the time of renal biopsy. All patients had a normal serum creatinine concentration ( $<120 \mu \mathrm{mol} / \mathrm{l}$ ) and normal blood pressure at the time of renal biopsy. Follow-up was 11 years median, with a range of 8 to 14 years. Progression of disease was defined as an increase in serum creatinine $(>140 \mu \mathrm{mol} / \mathrm{l}$, in the absence of therapy that reduces GFR), development of hypertension (diastolic blood pressure > $90 \mathrm{~mm}$ of $\mathrm{Hg}$ and systolic blood pressure $>140 \mathrm{~mm} \mathrm{Hg}$ ), increase in proteinuria (over $2 x$ initial proteinuria) or a combination of these three.

Renal biopsies were processed for light microscopy, immunofluorescence and ultrastructural analysis as described elsewhere [1]. Renal biopsies were classified according to the criteria as defined by the World Health Organisation [2]. Glomenular basement membrane thickness was determined by the method of Steffes et al [3]; using this method in adults a value of less than $260 \mathrm{~nm}$ is diagnostic for thin glomerular basal membranes [4]. The extent of focal global glomerulosclerosis in renal biopsies of all patients presenting with idiopathic haematuria was determined by counting the total number of glomeruli and the amount of sclerosed glomeruli in at least five consecutive $2 \mu \mathrm{m}$ sections, which were selected for their highest amount of glomenuli per section. stained with silver methenamin bematoxylin eosin.

Protein excretion was determined in a 24 -hour urine collection using the biuret method, and adequate sampling was verified by measuring creatinine excretion in an automated analyser based on the Jaffe reaction. Blood was drawn at the time of renal biopsy and at follow-up, allowed to clot for one hour at room temperature, and subsequently one hour on ice, serum was harvested and snap-frozen in liquid nitrogen. Serum samples were stored at $-70^{\circ} \mathrm{C}$ until usage. At the time of renal biopsy routine Jaboratory tests included antinuclear and rheumatoid factor, anti-streptolysin titre, 
classical haemolytic complement activity and liver function tests. In addition, in five patients with normal renal histology urinary calcium, phosphate, uric acid and creatinine excretion was determined. Hypercalciuria was said to exist when calcium excretion exceeded $4 \mathrm{mg} / \mathrm{kg} / 24 \mathrm{hr}$, hyperuricosuria was present if uric acid exceeded $800 \mathrm{mg} / 24 \mathrm{hr}$ in men, $750 \mathrm{mg} / 24 \mathrm{hr}$ in women [5]. Statistical analysis was performed on the SPSS 4.0 statistical package for the Apple computer.

\section{Results}

At the entry of the study 18 of the 49 patients were biopsied because of recurrent macroscopic haematuria; in the remaining 31 patients persistent microscopic erythrocyturia was the indication for renal biopsy.

Of the 49 patients who were biopsied $67 \%$ (33 patients) had isolated haematuria; their 24-hour protein excretion did not exceed the physiological limit ( $<180 \mathrm{mg} /$ day). The remaining sixteen patients had proteinuria that corresponded to 'trace' to ' + ' protein-excretion on the routine dipstick test, which in the 24 hour protein collection measured 180 to $500 \mathrm{mg} /$ day. After a median follow-up of 11 years haematuria was still present in 33 patients (10 patients with idiopathic IgA nephropathy, in 7 patients with normal renal tissue and in 3 patients with miscellaneous nephropathies). All patients with thin GBM nephropathy showed persistent microscopic haematuria at follow-up (Table 1). In IgA nephropathy resolution of haematuria occurred in two patients who had presented with persistent erythrocyturia. Both underwent repeat renal biopsy. Histologically "there was regression of the mesangial proliferation. Immunofluorescence analysis showed that the deposition of immunoglobulin $\lg A_{1}$ and complement $\mathrm{C} 3$ had disappeared, and on ultrastructural examination mesangial electron-dense deposits were no longer demonstrable. In the group with miscellaneous nephropathies haematuria disappeared during follow-up in one patient with active focal interstitial nephritis.

Of the patients with normal renal tissue $13(65 \%)$ spontaneously lost haematuria during follow-up. The median duration of persistent haematuria in nomal renal tissue was 53 months after renal biopsy. Remission of haematuria was equally distributed over the patients with macroscopic and microscopic haematuria, although in patients with macroscopic haematuria more symptoms of urolithiasis (passage of a stone, visible stone fragments in the urine, symptoms of renal colic) were encountered (Table 2). 
Table 1. Follow-up findings in patients presenting with chronic nonazotaemic non-proteinuric haematuria.

Immunopathological diagnosis

IgAN Thin GBM normal miscellaneous*

Initial presentation:

Number of patients

Malr fernale

Mean age (years)

Macroscopic haematuria

Microscopic haematuria

Proteinuria:

$-<180 \mathrm{mg} / \mathrm{day}$

- $180-500 \mathrm{mg} /$ day

12

11:1

13

20

4

30

8.5

$17: 3$

$2: 2$

61

612

30

44

10

12

10

1

3

Initial creatinine clearance

(mli/min) \#

109

115

113

93

Total number of

glomeruli/section

Focal global

glomerulosclerosis

Follow-up 11 years

Macroscopic haematuria

Microscopic haematuria

Hypertension

Increase in proteinuria

Creatimine clearance

(ml/min)

100

110

113

75 @

$\begin{array}{llll}5.0 \% \pm 6.8 & 9.6 \% \pm 13.7 \mathrm{a} \quad 2.6 \% \pm 4.2 \quad 4.0 \% \pm 4.9\end{array}$

$\begin{array}{llll}3 & 0 & 0 & 1 \\ 7 & 13 & 7^{*} & 2 \\ 5 & 7 & 1 & 3 \\ 4 & 5 & 2 & 2 \\ 100 & 110 & 113 & 75^{\Theta}\end{array}$

Legend to the table

${ }^{2}$ interstitial nephritis ( $\mathrm{x} 3$ ), focal global glomerulosclerosis $(\mathrm{x} 1)$

* renal calculi $(x 5)$, persistent erythrocyturia of unknown cause $(x 2)$

\# as determined using the Cockroft formula

a Mann Whitney $U$ test: $p=0,03$ thin GBM vs normal

b Mann Whitney U test: $p=0,002$ IgA ws. thin GBM, $p=0,03$ IgA vs. normal

@ Mann Whitney U test: $\mathrm{p}=0,006$ miscellaneous vs. normal 
At 11 year follow-up persistent haematuria was still present in seven patient with normal renal tissue. Five of these patients, of whom four were originally biopsied because of macroscopic haematuria, had symptoms suggestive of unolithiasis during follow-up. In two patients no cause was known for the haematuria (Table 2). Twentyfour hour urinary calcium, phosphate and uric acid excretion was determined in five of the seven patients with normal renal tissue who had a history of urolithiasis or persistent haematuria, and no hypercalciuria or hyperuricosuria could be demonstrated.

Table 2. Natural history of chronic idiopathic haematuria in 20 adults with normal renal tissue at renal biopsy and negative urological workup.

\begin{tabular}{lll} 
& $\begin{array}{l}\text { Macroscopic } \\
\text { haematuria }\end{array}$ & $\begin{array}{l}\text { Microscopic } \\
\text { haematuria }\end{array}$ \\
\hline Number of patients & 10 & 10 \\
Male:female ratio & $8: 2$ & $9: 1$ \\
Age at follow-up & $38 \pm 10.7$ & $46 \pm 11$ \\
Spontaneous resolution* & 6 & 7 \\
Renal calculi within 11 year & 4 & 1 \\
Persistent erythrocyturia & 0 & 2
\end{tabular}

Legend to the table

* Median duration of persistence of haematuria was 53 months

Hypertension was present in all patient groups after a follow-up period of 11 year (median). In thin GBM nephropathy 7 out of 13 (54\%) patients were hypertensive, in IgA nephropathy 5 out of $12(42 \%)$ and the miscellaneous group 3 out of 4. In three patients with thin GBM nephropathy, one patient with IgA nephropathy and 2 patients with miscellaneous nephropathies development of hypertension coincided with an increase in proteinuria. An isolated increase in proteinuria was found in two patients with thin GBM disease, three patients with IgA nephropathy, and two patients with normal renal tissue (subsequently, diabetes 
mellitus of late onset was diagnosed in one of them). Two patients with active interstitial nephritis showed decreased creatinine clearances at follow-up (Table 1). The incidence of hypertension was significantly higher both in the thin GBM nephropathy and IgA nephropathy patients, compared to patierts with normal renal tissue on biopsy, as only one patient in the latter group developed hypertension in the context of a positive family history for hypertension (Table 2). At follow-up all patients had a normal serum creatinine concentration.

Table 3. Incidence of hypertension in 49 patients presenting with chronic non-azotaemic non-proteinuric haematuria.

Diagnosis

N End follow-up

Hypertension Increased p-value

(+proteinuria) Proteinuria

$\begin{array}{lllll}\text { IgA nephropathy } & 12 & 5(1) & 3 & 0,019^{\mathrm{a}} \\ \text { Thin GBM } & 13 & 7(3) & 2 & 0,003^{\mathrm{b}} \\ \text { Miscellaneous } & 4 & 3(2) & 0 & 0,007^{\mathrm{c}} \\ \text { Normal } & 20 & 1 & 2 & \end{array}$

Legend to the table

The median follow-up was 11 years.

Hypertension is defined as RR $>140 / 90 \mathrm{~mm} / \mathrm{Hg}$, increased proteinuria is more than twic degree of proteinuria.

a Fisher exact test: IgA hypertensives vs. nomal renal tissue

b Fisher exact test: thin GBM hypertensives ws. normal renal tissue

C Fisher exact test: miscellaneous bypertensives vs. normal renal tissue

Because of the increased incidence of hypertension in thin GBM nephropathy renal biopsies were reassessed for histological changes. Apart from focal global glomerulosclerosis and sometimes local interstitial fibrosis and tubular atrophy, 
biopsies of thin GBM disease showed no histological changes. When the numbers of focally globally sclerosed glomeruli were counted in all renal biopsies, the $9.6 \%$ of sclerosed glomeruli which were found in thin GBM disease significantly exceeded that of controls (Table 1, Mann-Whitney-U test, $\mathrm{p}=0.03$ ). In IgA nephropathy the mean focal global glomerulosclerosis was $5 \%$, which was not significantly different from normal renal tissue, nor from thin GBM nephropathy. The increased presence of focal global glomerulosclerosis in thin GBM nephropathy was neither related to an increased total number of glomeruli ( $20 \pm 7$ glomeruli/section in thin GBM nephropathy, $17 \pm$ 11 glomenli/section in normal renal tissue; $\mathrm{p}=0.09$ ), nor caused by long standing hypertension as only one patient showed signs of renal microvascular abnormalities indicative of hypertension (intima fibrosis of the interlobar arteries and hyalinosis of the afferent arterioles). Focal global glomerulosclerosis was not secondary to interstitial nephritis, as none of the biopsies of thin GBM nephropathy showed interstitial nephritis. Nor was the focal global glomerulosclerosis in thin GBM disease related to age.

\section{Discussion}

In this study we report on the follow-up of 49 patients who underwent renal biopsy because of chronic non-azotaemic idiopathic haematuria with proteinuria less than $0.5 \mathrm{gram} /$ day ('trace' to ' + ' proteinuria on dipstick examination) and with a normal urological evaluation. From a previous study it was already known that the prevalence of IgA nephropathy and thin GBM nephropathy was identical in patients biopsied because of chronic haematuria [1]; in this report the prevalence was $24 \%$ and $26 \%$, respectively. This prevalence may differ between countries since it is influenced by the availability of transmission electron microscopy [6], and organization of the local health care including urine analysis for insurance, sports and draft registration. It has been shown that clinical findings at presentation are not belpful in the differential diagnosis of patients with chronic haematuria, as patients with urological causes of bleeding could not be distinguished from those with glomerular haematuria [4].

The role of renal biopsy in patients with non-proteinuric non-azotaemic haematuria is topic of debate, particularly after the introduction of urinary red cell morphology to differentiate between glomerular or non-glomerular haematuria. The initial claim [7] that the detection of dysmorphic erythrocytes by phase contrast microscopy could differentiate between glomerular and urological haematuria was 
refuted in a blind and controlled study [8]. A recent paper showed that of all the dysmorphic erythrocytes identified by phase contrast microscopy, only the acanthocyte, which is characterised by a ring form with vesicle-like protrusions, is indicative of glomenular bleeding, with a specificity of $98 \%$ and a sensitivity of $52 \%$ provided that over $5 \%$ of the urinary erythrocytes have this configuration [9].

As this acanthocyte is not inducible by changes in osmolarity, pH or urinary protein concentration it is useful clinically. In fact, in vittro work has demonstrated that passage of erythrocytes through the nephron together with some degree of haemolysis is required for acanthocyte formation [10]. However, patients with $\operatorname{IgA}$ nephropathy and patients with minor glomerular abnormalities, like thin GBM nephropathy, tend to have a low percentage of acanthocytes in the urine [9]. Therefore, renal biopsies remain necessary to differentiate chronic non-proteinuric glomenlar haematuria from urological causes.

In this study we found that the cause of haematuria corresponded well with the persistence of haematuria during follow-up, since haematuria remained present in almost all patients with glomerular or interstitial diseases. Only two patients with IgA nephropathy were shown to be in clinical, histological and ultrastructural remission; a finding described previously only in children with IgA nephropathy [11]. Conversely, in more than $65 \%$ of the patients with normal renal tissue haemanuria disappeared spontaneously during follow-up or was related to complaints suggestive of urolithiasis. We determined the excretion of calcium and uric acid in 24-hour urine collection of five patients who had symptoms of urolithiasis with persistent erythrocyturia of unknown origin, but were unable to demonstrate any hypercalciuria or hyperuricosuria. In the evaluation of hypercalciuria no measurement of citrate was obtained. The possibility that stone formation was caused by a low urine citrate concentration has not been excluded. Similarly, no information abont diet was obtained. It is well known that diet changes can reverse hypercalciuria and hyperunicosuria [5].

Our study shows that non-proteinuric thin GBM nephropathy and IgA nephropathy carry an excellent prognosis in relation to development of renal insufficiency, but are definite risk factors for the development of hypertension. Similarly, increased proteinuria (with or without development of hypertension) develops in $36 \%$ of the patients with glomerular haematuria, suggesting a slowly progressive course of disease. In contrast to patients with thin GBM nephropathy or IgA nephropathy, patients with interctitial nephritis show a significant decrease in creatinine clearance at the end follow-up, illustrating a faster progression of disease. 
How the hypertension in thin GBM disease is brought about is not known; the increased presence of focal global glomerulosclerosis suggests a renal origin. There is evidence that thin GBM nephropathy is slowly progressive in some patients, since they may develop renal insufficiency [12]. The high incidence of hypertension in thin GBM and IgA nephropathy is noteworthy considering the fact that the patients with chronic non-proteinuric haematuria are often not eligible for renal biopsy and subjected to prolonged follow-up on an outpatient basis [13], which in our and other"s [6] experience often leads to loss of the patient for medical evaluation or to repeated urological examinations.

As the median duration of persistence of haematuria in nomal renal tissue was four years it is advisable to perform renal biopsy on those patients in whom chronic non-proteinuric haematuria remains present for more than four years. In those patients nephrological causes of haematuria will be found in $86 \%$ of the biopsies. For adequate patient care it is important that these patients are diagnosed and checked regularly, as development of bypertension and/or proteinuria in the course of renal disease is usually an indication of slowly progressive renal disease and may contribute to development of renal failure. Therefore, we recommend that patients with idiopathic chronic and non-proteinuric haematuria, present for over four years, with normal renal function, blood pressure and a normal urological evaluation are subjected to renal biopsy to determine the cause of haematuria. When renal biopsy does not reveal any nephrological cause for haematuria patients should be under surveillance of an urologist, as they are liable to develop urolithiasis and occasionally malignancies [14]. 


\section{References}

1. Tiebosch A., Wolters J., Frederik $P_{\text {, }}$, van de Wiel $T$., Zeppenfeldi $\mathbb{E}$., van Breda Vriesman P. Epidemiology of idiopathic glomerular disease: A prospective study. Kidney Int 1987; 32:112-116.

2. Churg J., Bernstein J. Glassock R.: Renal Disease (second edition). TokyoNew York, Igaku-Shoin, 1995.

3. Steffes M. "Barbosa J., Basgen J., Sutherland D., Najarian J., Mauer S. Quantitative morphology of the normal human kidney. Lab Invest 1983; 49: 82-86.

4. Tiebosch A., Frederik P., van Breda Vriesman P., Mooy J., van Rie H., van de Wiel T., Wolters J., Zeppenfeldt $E$. Thin-basement-membrane nephropathy in adults with persisting hematuria. N Engl J Med 1989; 320: 14-18.

5. Andres A., Praga M., Bello L, Diaz-Colon J., Gutierrez-Millet V, Morales J., Rodicio J. Haematuria due to hypercalciuria and hypenuricosuria in adult patients. Kidney Int 1989; 36: 96-99.

6. Topham P., Harper S., Furness P., Harris K., Walls I., Feehally J. Glomerular disease as a cause of isolated microscopic haematuria. Q J Med $1994 ; 87: 329-335$.

7. Birch D., Fairley K. Hematuria: glomerular or non-glomerular? Lancet 1979; 845-846.

8. Venkat Raman G., Pead L., Lee H., Maskell R. A blind controlled trial of phase-contrast microscopy by two observers for evaluating the source of haematuria. Nephron 1986; 44: 304-308.

9. Kóhler H., Wandel E., Brunck B. Acanthocyturia - A characteristic marker for glomerular bleeding. Kidney Int 1991; 40:115-120.

10. Schramek P., Moritsch A., Haschkowitz H., Binder B., Maier M. In vitro generation of dysmorphic erythrocytes. Kidney Int 1989; 36: 72-77.

11. Yoshikawa N., Jjma K., Matsuyama S., Suzuki I., Kameda A., Osada S., Nakamura H. Repeat renal biopsies in children with IgA nephropathy. Clin Nephrol 1990; 33: 160-167.

12. Dische F., Weston M., Parsons V. Abnornally thin glomerular basement membranes associated with hematuria, proteinuria or renal failure in adults. Am J Nephrol 1985; 5: 103-109. 
13. Schramek P., Georgopoulos M., Schuster F., Porpaczy P. Value of urinary erythrocyte morphology in assessment of symptomless microhaematuria. Lancet 1989; 1316-1319.

14. Rasmussen O., Andersen J., Olesen E., Dimo B. Recurrent unexplained haematuria and risk of urological cancer. Scand J Urol Nephrol 1988; 22: 335337. 



\section{Chapter 3.}

\section{Thin GBM nephropathy: premature glomerular}

obsolescence is associated with hypertension and late onset renal failure 


\section{Summary}

Thin glomerular basement membrane (GBM) nephropathy, also called familiall benign haematuria, is characterised by chronic haematuria and uniform thinning of the lamina densa of the glomerular basement membrane. It generally holds an excellent renal prognosis. Alport syndrome in early stages can also show attenuation of the GBM; conversely, renal insufficiency has been reported in familial benign haematuria. In order to discem early Alport syndrome from thin GBM nephropathy we carried out a prospective epidemiological study in which 19 normotensive and non-azotemic adult patients with chronic microscopic (18 of 19) and macroscopic ( 1 of 19) haematuria and biopsy-proven thin GBM nephropathy were followed for 12 years median (range 9 to 15 years).

Renal biopsies of thin GBM patients at entry showed an increased incidence of focal global glomerulosclerosis when compared to disease controls as IgA nephropathy ( $p=0.047)$ and normal renal tissue $(p=0.075)$. All renal biopsies showed presence of the Goodpasture antigen when tested immumohistochemically. Presence of Alport syndrome was excluded clinically as none of the patients had complaints of hearing loss or abnormalities by audiography and ophthalmology. At the end of follow-up, the incidence of hypertension in thin GBM nephropathy (35\%) exceeded that of healthy clinical controls $(\mathrm{p}=0.048$ ), one of the hypertensives developed mild renal failure. In the normotensive patients, the glomerular filtration rate at follow-up as measured by inulin clearance, was reduced in 3 out of 7; these were over 50 years of age. Although no family members were known to have renal disease at inclusion, within four families six elderly first degree relatives had developed unexplained renal insufficiency at the end of follow-up. Thus, thin GBM nephropathy predisposes to premature glomerular obsolescence leading in time to increased incidences of hypertension and late onset renal insufficiency. 


\section{Introduction}

Thin glomerular basement membrane (GBM) nephropathy, so-called benign familial haematuria, refers to patients with chronic haematuria, usually of the microscopic variety, is equally distributed over the sexes, and carries an excellent short-term renal prognosis [1]. The diagnosis is made by means of quantitative ultrastructural morphometry which shows a uniform thinning of the lamina densa of the glomerular basement membranes. Silver impregnation staining of these biopsies reveals thinning, local reduplication and a moth-eaten mesangial matrix, all considered to be minor glomerular changes (Figure 2c) [2]. Clinically, thin GBM nephropathy and the progressive renal hereditary disorder Alport syndrome appear distinct entities, although in individual cases, particularly in children, the differentiation may be difficult as the histological changes cited above may also be seen in early Alport syndrome [3]. Also, renal insufficiency has been observed occasionally in thin GBM nephropathy [4, 5]. In Alport syndrome, different clinical manifestations are recognised and juvenile and adult-onset renal insufficiency are distinguished [6]. The mode of inheritance is most often $\mathrm{X}$-linked, occasionally autosomal recessive or, rarely, autosomal dominant. In Alport syndrome immunohistochemical studies have implicated collagen type $\mathrm{IV}$ in the pathogenesis and several causative mutations have been identified in different type IV collagen genes (reviewed in [7]) Recently, Lemmink et al. $[8]$ detected a missense mutation in collagen type $N$ in benign familial haematuria, which raises the question whether or not thin GBM nephropathy is an atypical form of Alport syndrome. We addressed this question by long-term follow-up of normotensive adults with thin GBM nephropathy and appropriate clinical controls in a prospective epidemiological study on idiopathic haematuria. None of the patients were known to have familial renal disease at entry into the study, but follow-up showed most of the thin GBM nephropathy patients to have familial haematuria with an increased incidence of renal insufficiency in first degree relatives.

\section{Patients}

Patients were derived from a prospective epidemiological study of idiopathic glomerular disease carried out between 1978 and 1984 [1]. Briefly, renal biopsies were taken from patients between 16 and 65 years of age. provided systemic extrarenal disease or known farnilial renal disorders were absent. The indications for renal 
biopsy included, in herarchical order: recent onset uraemia (serum creatinine $>300$ $\mu$ mol/1), recent onset nephrotic syndrome (proteinuria $>5$ gram/day, in the absence of Bence Jones proteinuria), recent onset haematuria with proteinuria and mild azotaemia, chronic non-urological haematuria of the persistent microscopic or recurrent macroscopic variety with or without proteinuria $(>250 \mathrm{mg} /$ day), chronic proteinuria in the absence of hypertension. In the present study, renal biopsies were taken from normotensive non-azotaemic patients with idiopathic chronic haematuria of six months duration. Twenty-seven patients were shown to have primary IgA nephropathy, 24 patients had normal renal tissue and 19 patients were diagnosed as thin glomerular basement nephropathy patients. Patients were biopsied because of non-urological microscopic or macroscopic haematuria, in the absence of gross proteinuria. Biopsies were taken within two years after patient identification. Persistent erythrocyturia was defined as the consistent presence of more than 5 erythrocytes per high power field of centrifuged urine. All patients had a normal blood pressure, none had increased serum creatinine concentrations and in all patients urologic examination was negative. Patients with systemic disease, Henoch Schönlein nephritis, liver disease or patients from families with known hereditary nephritis were excluded from the study. None of the patients with thin GBM disease was consanguineous or had knowledge of farnilial haematuria at the time of renal biopsy. All patients were followed for a median period of 12 years (range 9 to 15 years). Signs of disease progression were development of hypertension ( $>140 / 90 \mathrm{~mm} \mathrm{Hg}$ ), increase in proteinuria (at least $0.5 \mathrm{~g} /$ day at followup), or development of uraemia (serum creatinine concentration $>140 \mu \mathrm{mol} /$ ). In three patients repeat renal biopsies were taken to determine the histological substrate for disease progression.

\section{Methods}

\section{Renal biopsies}

Renal biopsies were processed for light microscopy, immunofluorescence and ultrastructural analysis as described elsewhere [9]. Renal biopsies were classified according to the criteria as defined by the World Health Organization [3]. Glomerular basement membrane thickness was determined by the method of Steffes et al [10]; using this method in adults a value of less than $260 \mathrm{~nm}$ is diagnostic for thin glomerular basement membranes [1]. The extent of focal global glomerulosclerosis in renal biopsies of all patients presenting with idiopathic haematuria was determined by 
counting the totall number of glomeruli and the amount of sclerosed glomerull in at least five consecutive $2 \mu \mathrm{m}$ sections - were selected for their highest amount of glomeruli per section (range of glomeruli per section 12-41) - stained with silver methenamin hematoxylin eosin. In addition, $2 \mu \mathrm{m}$ thick cryosections were stained in an indirect immunofluorescence assay for presence of the putative Goodpasture antigen with a mouse monoclonal antibody (mAb 17) against the non-collagenous domain of the $\alpha 3$-chain of collagen type 4 (kindly provided by J. Wieslander, Biocarb, Denmark). Binding of the monoclonal antibody was visualised with a 1 to 100 dilution of a polyclonal goat anti-mouse Ig-FITC-labelled antibody (Organon Teknica Cappel, Boxtel, The Netherlands). As positive controls, sections of normal renal tissue were tested.

Tone and speech audiograms were obtained from 10 thim GBM patients, performed by a trained audiometrist. These patients were also subjected to a slit-lamp and fundoscopy examination performed by a trained ophthalmologist. Development of hypertension and decreased GFR was equally divided over the patients who were tested with audiograms and those who were not tested. Proteinuria was determined at initial biopsy and at follow-up in a 24-hour urine specimen. Protein content was measured with a biuret assay, and adequate sampling was verified by measuring creatinine excretion in an automated creatinine analyser based on the Jaffe reaction. When patients had proteinuria in excess of $200 \mathrm{mg} /$ day, orthostatic proteinuria was ruled out by overnight collections. Aliquots of urine were dialysed against water, lyophilised and stored at $4^{\circ} \mathrm{C}$. When desired, equal fractions of this lyophilised urine were subjected to gradient electrophoresis in order to determine the type of proteinuria.

\section{Renal function studies}

Creatinine clearances were calculated using the Cockroft formula ${ }^{3}$, while GFR and ERPF values were determined using a continuous infusion of inulin and paraamino-hippuric acid. For determination of GFR and ERPF patients were examined in a supine position and adequate diuresis was ensured by oral intake of 200 to $300 \mathrm{ml}$ water per hour. A blood blank was drawn and a priming dose of $500 \mathrm{mg} / \mathrm{m}^{2}$ PAH (Aminohippurate sodium, MSD, West Point, USA) and $1000 \mathrm{mg} / \mathrm{m}^{2}$ inulin (Inutest, Laevosan Gesellschaft, Linz, Austria) was prepared, of which $80 \%$ was given in a bolus and the remainder at a flow rate of $100 \mathrm{ml} / \mathrm{hour}$. This was followed by a

The Cockroft formula as employed: GFR = (140-age $)^{*}$ weight/serum creatinine ( $\mathrm{mg} / \mathrm{dl}$ )*72, for females the result was multiplied with 0.85 . 
continuous infusion of PAH and inulin, while the infusion rate was adapted to the estimated renal function. An equilibration time of 120 minutes was used, and subsequently four blood samples were drawn every 10 minutes. PAH was determined spectrophotometrically according to Brun [11], inulin was determined spectrophotometrically as glucose after full enzymatic hydrolysis of fructose [12]. We only tested the normotensive thin GBM patients to avoid confounding of the effects of hypertension and therapy for hypertension on GFR and ERPF.

\section{Statistics}

Statistical callculations were performed on a SPSS programme for the Apple computer. When absolute numbers were compared, the chi square test with the Fisher exact correction was used. Comparison between IgA nephropathy, thin GBM nephropathy and normal renal tissue were performed using the Mann Whitney rank test.

Table 1. Family history in thin GBM nephropathy and primary IgA nephropathy

\section{Thin GBM nephropathy IgA nephropathy}

Family history available in

Number of parents

:of which in renal failure

Number of siblings

:of which in renal failure

Total first degree relatives

of which in renal failure*
16

32

3

57

3

89

6
25

50

1

79

0

129

1

Legend to the table

Renal failure was defined as serum creatinine concentration over $300 \mu \mathrm{mol} / \mathrm{h}$

\#Fisher exact test: $\mathrm{p}=0,019$ 


\section{Results}

\section{Family history in thin GBM nephropathy}

Af the time of renal biopsy all patients with thin GBM nephropathy were considered to be isolated cases of idiopathic chronic haematuria, but caneful history taking and family research at follow-up revealed that haematuria, proteinuria and/or impaired renal function was present in the families of 9 of 16 patients (in three cases no data on the family history was available). Within four families, six elderly first degree relatives of the index patients were identified who had end-stage renal disease necessitating dialysis (Table 1). Of these elderly family members, aged between 55 and 67 years, no renal biopsy specimens were available. At the time of presentation they all had moderate to severe renal failure with microscopic haematuria, proteinuria and hypertension. The mode of inheritance in these familles appeared to be autosomal dominant (figure 1). Similarly, the family history in $25 \mathrm{IgA}$ nephropathy patients who were biopsied for idiopathic haematuria was examined. A positive family history for renal failure was present in one instance, while familial haematuria was identified in another family (Table 1). Of the 24 patients with normal renal tissue, none had family members with renal insufficiency.

\section{Follow-up of thin GBM nephropathy}

During the 12 year follow-up period two patients had died due to pancreatic and lung carcinoma, and therefore no renal data were obtained from them. Nine patients showed clinical signs suggestive of progressive renal disease: 7 patients developed hypertension, associated with increased proteinuria in 2, and mild renal insufficiency (creatinine concentration of $150 \mu \mathrm{mol} /$ ) in 1 . Two other patients developed increased proteinuria (of 0.6 and $1.6 \mathrm{~g} / \mathrm{day}$ ) in the absence of hypertension. In all but one patient with thin GBM nephropathy, proteinuria was of the non-selective variety, both at the time of renal biopsy and at the end of follow-up. Of the hypertensives, six of seven were male, while increased proteinuria was seen 2 of the eight females. In the normal renal tissue group, two patients developed essential hypertension with a positive family history hypertension; one patient developed increased proteinuria, which preceded the diagnosis of type II diabetes mellitus (Table 2). The incidences of hypertension and increased proteinuria were comparable to those in IgA nephropathy. However, in IgA nephropathy three patients progressed to renal failure, necessitating renal replacement therapy in one. Creatinine clearances, in the thin GBM group, calculated by the Cockroft formula showed no significant change during the follow. 
Table 2. Clinical presentation and follow-up in thin GBM nephropathy and hematuric controls.

$\begin{array}{lll}\text { Thin GBM } & \text { IgA } & \text { Normal } \\ \text { nephropathy } & \text { nephropathy } & \text { renal tissue }\end{array}$

Clinical presentation:

Number of patients

$$
19
$$

36

Male:female ratio

-Isolated haematuria

-Haematuria + proteinuria*

Histology at entry:

-Total glomeruli/section

-Focal global glomerulosclerosis

-GBM thickness (nm)
$18 \pm 7$

$13.5 \pm 17 \%$

$191 \pm 28$
27

31

20:7

7

20

$14 \pm 8^{\#}$

$5.1 \pm 7.3 \% \&$

$345 \pm 55$

27

$8(3)$

3

3(1)
24

32

$21: 3$

18

6

\section{Follow-up:}

No. patients

-Hypertensivell

-Increased proteinuria

-Increased serum creatininefl

170

6(1)

2

1

$\begin{array}{ll}7 & 24 \\ (3) & 2(0) \\ & 1 \\ (1) & 0\end{array}$

$16 \pm 10$

$3.1 \pm 4.4 \%$

$361 \pm 69$

Legend to the table

\#Protein excretion $>250 \mathrm{mg} /$ day

\# Total number of glomeruli: IgA nephropathy vs. thin GBM nephropathy: $\mathrm{p}=0.059$; IgA nephropathy vs. normal renal tissue: $p=0.17$; thin GBM nephropathy vs. normal renal tissue: $p=0.37$.

\& Focal global glomerulosclerosis: thin GBM nephropathy vs. IgA: $\mathrm{p}=0.047$; thin GBM nephropathy vs. normal renal tissue: $\mathrm{p}=0.0075$.

a Two patients with thin GBM nephropathy had died during follow-up due to pancreatic and lung carcinoma.

II Between parenthesis are mentioned the proteinuric hypertensives.

II Increased serum creatinine concentration over $140 \mu \mathrm{mol} / 1$, between parenthesis are mentioned the end-stage renal failure patients 
up. (creatinine clearance at initial biopsy $98 \pm 18 \mathrm{ml} / \mathrm{min} / 1.73 \mathrm{~m}^{2}$, at follow-up $92 \pm 17$ $\left.\mathrm{ml} / \mathrm{min} / 1.73 \mathrm{~m}^{2}\right)$. GFR and ERPF were determined in seven of 10 patients who had remained normotensive at the end of follow-up. GFR was shown to be reduced in 3 patients over 50 years of age when compared to age matched controls. Similarly, ERPF was slightly lower than expected in these patients compared to normal controls (Figure 2). In the initial renal biopsies of thin GBM nephropathy an increased percentage of focal global glomerulosclerosis, when compared to normal renal tissue controls, was already present (Table 2), which was confirmed in the repeat renal biopsies that were taken from three patients who had shown development of hypertension ( $1 \mathrm{x})$, increase in proteinuria $(1 \mathrm{x})$ or development of renal insufficiency (1x). In these repeat renal biopsies increased focal global glomerulosclerosis with tubular atrophy and interstitial fibrosis was present, without lesions of active interstitial infiltrate, focal and segmental glomerulosclerosis or foam cells (Figure 2b). Persistent thinning of the GBM in an unimodal distribution was present ultrastructurally, with only local reduplication and splitting, histologically.

Table 3. GFR and ERPF measurements in normotensive thin glomerular basement nephropathy patients at the end of follow-up

Thin GBM nephropathy

\begin{tabular}{|c|c|c|c|c|c|c|}
\hline \multirow{2}{*}{ no. } & \multirow{2}{*}{$\begin{array}{l}\begin{array}{l}\text { sex/age } \\
(\mathrm{yr})\end{array} \\
\mathrm{f} / 35\end{array}$} & \multicolumn{3}{|c|}{$\begin{array}{l}\text { GFR ERPF FF } \\
\left(\mathrm{ml} / \mathrm{min} / 1.73 \mathrm{~m}^{2}\right)\end{array}$} & \multicolumn{2}{|c|}{$\frac{\text { GFR ERPF }}{(\mathrm{ml} / \mathrm{min} / 1.73 \mathrm{~m})}$} \\
\hline & & 117 & 531 & 0.22 & \multicolumn{2}{|c|}{ (age $33-47$ years, $n=11$ ) } \\
\hline no. 2 & $\mathbb{1} / 38$ & 116 & 409 & 0.28 & $113 \pm 11$ & $414 \pm 70$ \\
\hline no. 3 & $1 / 38$ & 96 & 429 & 0.22 & & \\
\hline no. 4 & $\$ / 48$ & 102 & 358 & 0.28 & \multicolumn{2}{|c|}{ (age $52-67$ years, $n=6$ ) } \\
\hline no. 5 & $\mathrm{f} / 5 \mathrm{I}$ & 79 & 322 & 0.25 & $97 \pm 13$ & $404 \pm 59$ \\
\hline no. 6 & $\mathrm{f} / 58$ & 79 & 357 & 0.22 & & \\
\hline no. 7 & $\mathrm{~m} / 6 \mathrm{i}$ & 64 & 251 & 0.25 & & \\
\hline
\end{tabular}

* GFR and ERPF are determined using inulin and para-aminohippuric acid clearances. 


\section{Differentiation from Alport syndrome}

None of the patients with thin GBM nephropathy suffered from subjective hearing loss or vision problems. Audiograms and ophthalmologic examination showed. that none of the patients had signs of sensorineural hearing loss, anterior lenticonus or macular changes. Furthermore, all renal biopsies of the thin GBM patients were stained for the presence of the NCl-domain of the a3-chain of collagen type $\mathrm{TV}$. All patients showed diffuse intense glomerular capillary linear staining with variable intensity of tubular staining, illustrating presence of the Goodpasture antigen. The staining pattern was similar to that seen in eighteen patients with normal renal tissue on biopsy.

\section{Discussion}

The main findings of this study were fourfold. First, field studies showed the majority of the patients with apparently non-familial erythrocyturia to be in fact of the familial variety. Second, thin GBM nephropathy was associated with a significantly higher incidence of renal insufficiency in the first degree relatives and with an increased incidence of focal global glomerulosclerosis when compared patients with idiopathic IgA nephropathy and with normal renal tissue associated with haematuria. Third, twelve year follow-up revealed an incidence of hypertension comparable to that in patients with idiopathic non-azotemic $\operatorname{IgA}$ nephropathy, and showed a decline in renal function in three elderly patients. Finally, none of the thin GBM patients developed sensorineural hearing loss.

The familial nature of haematuria in thin GBM nephropathy has been described previously $[2,4,5,13,17]$. Since the haematuria is usually of the microscopic variety and renal failure occurs only in elderly family members, none of the thin GBM patients in the present study, of whom the mean age at entry was 36 years, was aware of the familial nature at the time of renal biopsy. It should be noted that of our thin GBM patients five presented with significant proteinuria ( $>250 \mathrm{mg} / \mathrm{day}$ ), while this increased during follow-up in another two. The origin of the increase in proteinuria in the absence of hypertension is not clear. In some of the thin GBM nephropathy patients, not belonging to the present series, increased proteinuria is associated with the presence of focal and segmental glomerulosclerosis in the renal biopsy (unpublished observation). Renal failure, either in patients with thin GBM nephropathy or in first degree relatives has only been reported occasionally $[4,5]$, but 
the high incidence of renal insufficiency in elderly first degree relatives as seen in our study has not been described previously. We could not find an increased prevalence of renal disease in our population of $\operatorname{Ig} A$ nephropathy patients, contrary to experience of others [18].

During follow-up hypertension developed in more than one third of the patients, while proteinuria increased in an additional two. Interestingly, although males and females were equally affected by thin GBM nephropathy, hypertension was more prevalent in males while increased proteinuria occurred only in femalles. This predominance of males with hypertension was not seen in our patients with IgA nephropathy, but corresponds to the findings in both a large study of hypertension in patients with glomerulonephritis [19] and to the increased prevalence of hypertension in men in the general population [20]. In a recent study of haematuric patients with thin and ultrathin GBM, in which the criteria for diagnosis of thin GBM nephropathy were different from our study, $14 \%$ presented with hypertension, while $28 \%$ had proteinuria. [21] Reduced renal function was found in three nomotensive thin GBM palients over 50 years of age as illustrated by a decreased GFR and ERPF, one other patient showed an increased serum creatinine concentration. We postulate that the increased incidence of hypertension, development of proteinuria and decreased GFR and ERPF is related to the increased degree of focal global glomerulosclerosis as seen in the initial biopsy and repeat renal biopsies of the thin GBM patients. The fact that decreases in GFR and ERPF were seen in patients who had remained normotensive, suggest that a renal substrate is responsible for the loss in renal function. Histologically, focal global glomerulosclerosis with tubular atrophy and interstitial fibrosis appears a likely substrate. Thus, the prognosis of thin GBM nephropathy may not be as benign as generally thought $[2,13]$ and, given the incidence of the disease in The Netherlands [9], may be a cause of end-stage renal disease in a significant portion of elderly patients.

Clinically, thin GBM nephropathy and Alport syndrome appear distinct entities, although in individual cases, especially in children, the differentiation between Alport and thin GBM nephropathy can be difficult since Alport syndrome can present with uniform thinning of the GBM. In Alport syndrome, different clinical manifestations are recognised, and juvenile and adult onset end-stage-renal disease are distinguished. The juvenile form is said to exist if end-stage-renal-disease occurs before 31 years of age. Atkin, Gregory and Border [6] classified the disease in six types with dominant inheritance: type I is dominant juvenile Alport associated with deafness, either Xlinked or autosomal, type II is $\mathrm{X}$-linked dominant juvenile associated with deafness, 
type III is X-linked dominant adull associated with deafness, type $\Gamma V$ is X-linked adult without deafness or other extrarenal symptoms, type $V$ is autosomal with deafness and macrothrombocytopathy and type VT is autosomal dominant juvenile with deafness. For the diagnosis of Alport syndrome four criteria have been defined, three of which have to be present to allow the diagnosis: (1) positive family history of haematuria or chronic renal failure, (2) electron microscopic evidence of Alport syndrome on renal biopsy, (3) progressive high tone sensorimeural deafness and (4) characteristic eye symptoms [22]. Our thin GBM nephropathy patients fulfilled only one or two criteria of this classification. Furthermore, in all our patients the $\alpha 3$ collagen type IV chain was demonstrable in immunofluorescence studies of frozen biopsies. Repeat renal biopsies in thin GBM nephropathy, taken after a median disease history of 10 years, showed persistent thinning of the GBM in a unimodal distribution, with only local reduplication and splitting; foam cells were not seen in these biopsies. Thus, thin GBM nephropathy resembles Alport syndrome in terms of chronic, often familial, erythrocyturia, but lacks the extra-renal symptoms of Alport syndrome. It may share some of the ultrastructural GBM features, but does not fulfil the diagnostic criteria for Alport syndrome.

Biopsies of thin GBM nephropathy show extreme attenuation of the lamina densa, which is built up from collagen type TV, a triple helix collagen incorporating differing combinations of the $\alpha 1($ TV)- $\alpha 5$ (IV) chains. The genes involved in collagen type IV synthesis are therefore good candidates for genetic analysis. In the X-linked form of Alport syndrome, mutations in the COLAA5 gene have been described, (reviewed in [7]). In the autosomal recessive form of Alport syndrome, mutations in the COL4A3/COLAA4 have been identified, where several homozygous mutations resulted in either prematurely teminated collagen chains or in interruption of the Glly$X$-X-repeat, thus interfering with the aggregation of the collagen type 4 trimer. [23] Savige et al. [16] excluded linkage of thin GBM nephropathy with the collagen type IV $\alpha 1$ and $\alpha 2$ chains. Lemmink et al., using intragenic COLAA3/COLAA4 markers, recently detected a missense mutation in the COL $4 A 4$ gene in a family with benign familial haematuria, of which the propositus, a 6 year old boy, showed focal and segmental GBM abnormalities, typical for Alport syndrome, and thinning of the GBM [8].

Thus, although thin GBM nephropathy is clinically different from Alport syndrome, both thin GBM nephropathy and Alport syndrome appear to result from defects in collagen type TV coding genes. Hence, thin GBM nephropathy predisposes 
to premature glomerular obsolescence, hypertension and increased incidence of late onset renal insufficiency.

Legend to figure 1.

Two family pedigree depicting the mode of inheritance in thin GBM nephropathy. The open symbols or '?' represent untested individuals, the unaffected individuals are indicated by a ' $n$ '. The hematuric individuals are indicated with a semi-filled symbol, while hematuric and proteinuric individuals are indicated with a three-quarterly filled symbol. Renal insufficiency is indicated with a black symbol, the deceased individuals are represented with a slash through the symbol. Squares represent males, while circles represent females. Arrows refer to renal biopsy-proven thin GBM nephropathy. 
Chapter 3

Figure 1. Family trees of two families with thin GBM nephropathy, biopsy-proven in the index patients

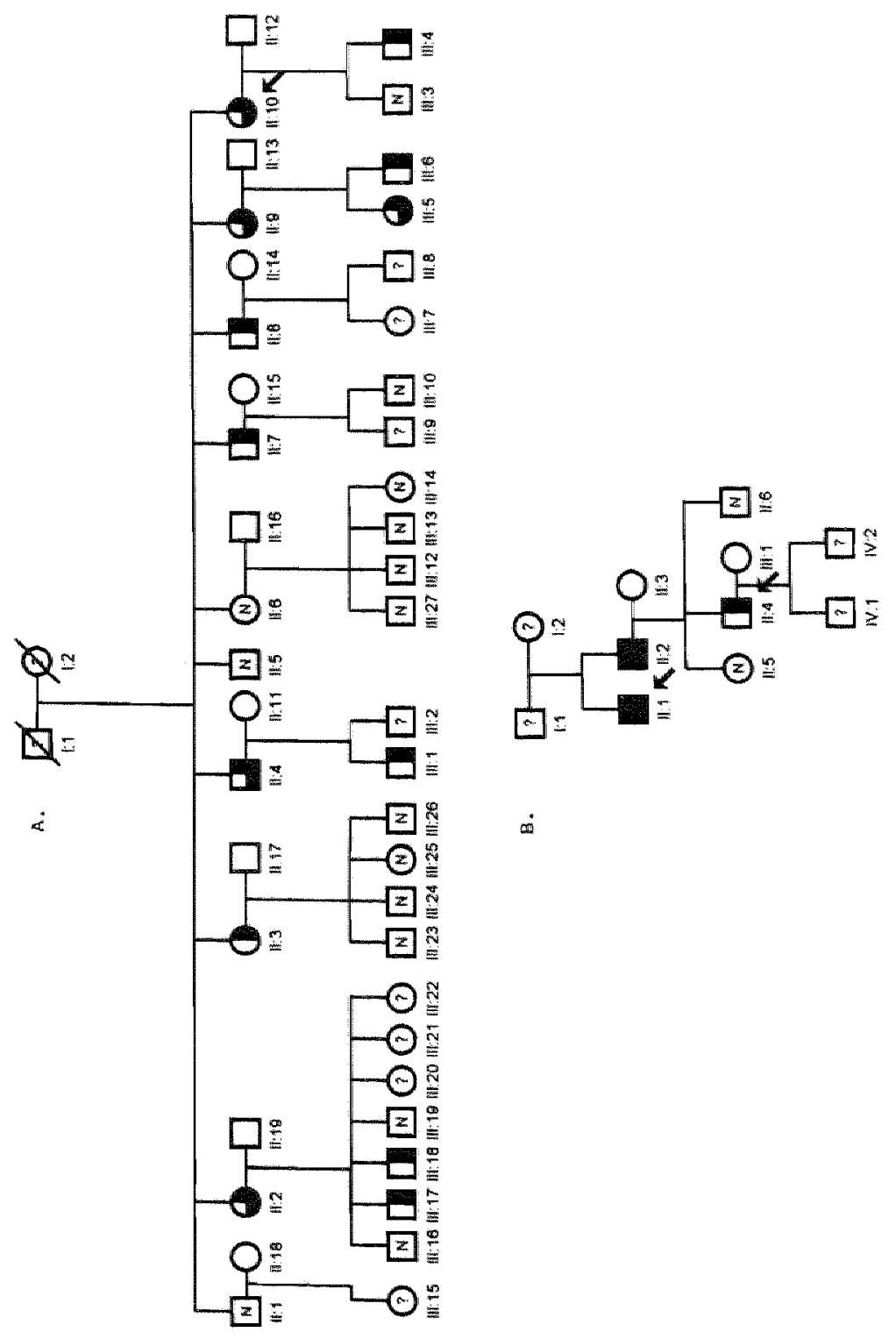


Figure 2. GFR (A) and ERPF (B) in normotensive thin GBM nephropathy

a. GFR measurements
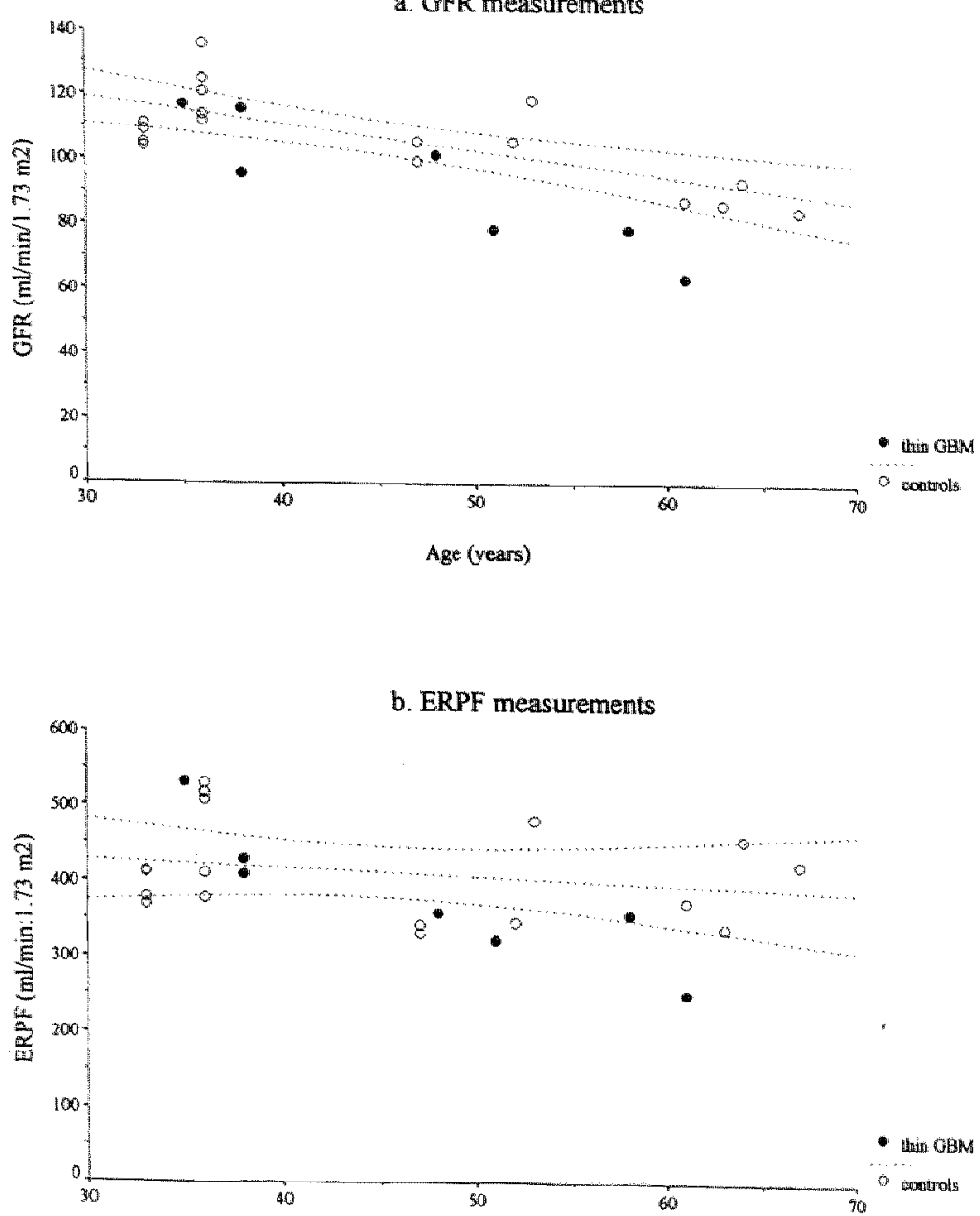

Legend to figure 2.

Determination of GFR (figure 2a) and ERPF (figure $2 b$ ) in normotensive thin GBM nephropathy, as measured with inulin and PAH clearances, compared to healthy subjects. Symbols are: (o) GFR and ERPF measurements in healthy subjects between 33 and 67 years of age; ( $)$ GFR and ERPF data in the normotensive thin GBM patients that were available for renal function studies. GFR is reduced in three elderly thin GBM nephropathy patients, in one patient ERPF also clearly is lower than in the nomal subjects. The middle line represents the linear regression line, the outer lines represent the upper and lower $95 \%$ confidence intervals. Multiple regression analysis showed the GFR regression line of thin GBM patients to differ significantly from the healthy controls $(p=0.022)$; the ERPF regression lines are not different $(p=0.37)$. 
Figure 3. Histology in thin GBM nephropathy
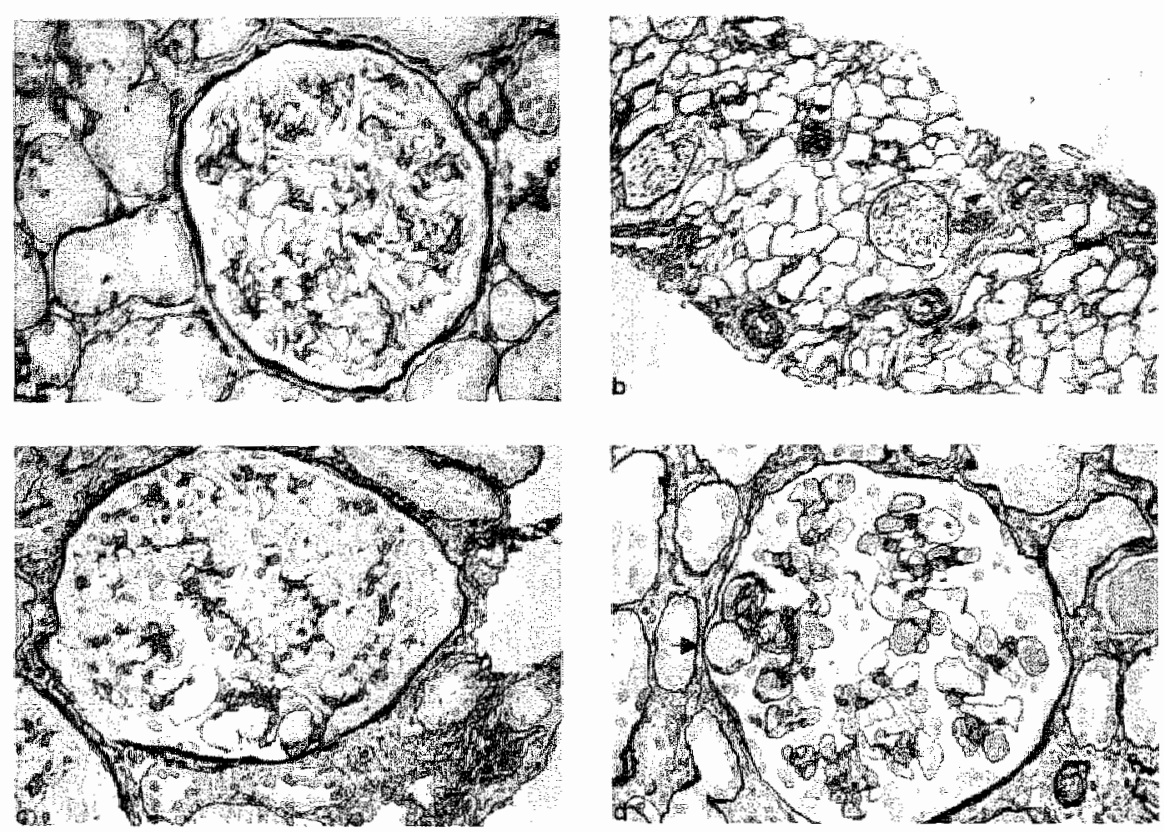

Legend to figure 3.

A. Normal glomerulus (silver methenamin hematoxylin eosin stain). Note the silverimpregnation of the GBM in all capillaries which is comparable to the staining of the tubular basement membrane (160x).

B. Thin GBM nephropathy, silver methenamin hematoxylin eosin stainin (160x). Repeat renal biopsy, taken because of hypertension and increased serum creatinine. Note the diffuse focal global glomerulosclerosis, as indicated with arrow-heads, tubular atrophy and interstitial fibrosis.

C. Thin GBM nephropathy, glomerulus with minimal abnormalities besides abnormal silver-impregnation of the GBM (arow, silver methenamin hernatoxylin eosin stain). The diagnosis thin GBM nephropathy is made upon measurement of the GBM thickness in ultrathin sections $(160 \mathrm{x})$.

D. Thin GBM nephropathy with classical lesions of focal and segmental hyalinosis and sclerosis (arrow) (160x). 


\section{References}

1. Tiebosch A., Frederik P., van Breda Vriesman P., Mooy J., van Rie H., van de Wiel T., Wolters J., Zeppenfeldt $\mathrm{E}$. Thin-basement-membrane nephropathy in adults with persisting hematuria. N Engl J Med 1989; 320: 14-18.

2. Saxena $S$., Davies D., Kirsner $R$. Thin basement membranes in minimally abnormal glomerulli. J Clin Pathol 1990; 43: 32-38.

3. Churg J., Bernstein I., Glassock R.: Renal Disease (second edition). Tokyo-New York, Igaku-Shoin, 1995.

4. Trachiman H., Weiss R., Bennett B., Greifer I. Isolated hematuria in children: indications for a renal biopsy. Kidney Int 1984; 25: 94-99.

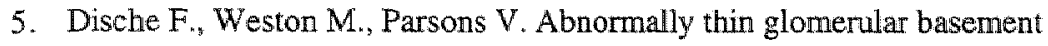
membranes associated with hematuria, proteinuria or renal failure in adults. Am J Nephrol 1985; 5: 103-109.

6. Atkin CL., Gregory M., Border W.: Alport syndrome Boston/Toronto, Little, Brown and Company, 1988.

7. Tryggvason K., Zhou J., Hostikka S., Shows T. Molecular genetics of Alport syndrome. Kidney Int 1993; 43: 38-44.

8. Lemmink H., Nilesen W., Mochizuki T., Schröder C., Brunner H., van Oost B., Monnens L., Smeets H. Benign familial hematuria due to mutation of the Type IV collagen $\alpha 4$ gene. J Clin Invest 1996; 98: 1114-1118.

9. Tiebosch A., Wolters J., Frederik P., van de Wiel T., Zeppenfeldt E., van Breda Vriesman P. Epidemiology of idiopathic glomerular disease: A prospective study. Kidney Int 1987; 32: 112-116.

10. Steffes M. , Barbosa J., Basgen J., Sutherland D., Najarian J., Mauer S. Quantitative morphology of the nomal human kidney. Lab Invest 1983; 49: 8286.

11. Brun C. A rapid method for the determination of para-aminohippurin acid in kidney function test. J Lab Clin Chem 1951; 37: 955-98.

12. Zittan L. Enzymatic hydrolysis of inulin. An altemative way to fructose production. Storch/Stärke 1981; 33: 5373-5377.

13. Lancet. Benign familial haematuria. Editorial. Lancet 1988; 549-550.

14. Schröder C., Bontemps C., Assmann $\mathbb{K}$, Schuurmans Stekhoven J., Foidart J., Monnens L., Veerkamp J. Renal biopsy and family studies in 65 children with isolated hematuria. Acta Paediatr Scand 1990; 79: 630-636. 
15. Lang S., Stevenson B., Risdon R. Thin basement membrane nephropathy as a cause of recurrent haematuria in childhood. Histopathol 1990; 16: $331-337$.

16. Savige J. Hereditary abnormalities of renal basement membrane. Pathology 1991 ; 23: 350.355 .

17. Bodziak K., Hammond W., Molitoris B. Inherited diseases of the glomerular basement membrane. Am J Kid Dis 1994; 23: 605-618.

18. Schena $F_{\text {, }}$ Scivitarro V., Ranieri E. IgA nephropathy, pros and cons for a familial disease. Contr Nephrol 1993; 104: 34-45.

19. Johnston P., Dawison A. Hypertension in adults with idiopathic glomerulonephritis and normal serum creatinine. A report from the MRC Glomerulonephritis Registry. Nephrol Dial Transplant 1993; 8: 20-24.

20. Kaplan: Clinical Hypertension (Sixth Edition). Baltimore, Williams and Wilkin, 1994.

21. Goel S., Davenport A., Goode N., Shires M., Hall C., Harrison P., Maciver A. Clinical features and outcome of patients with thin and ultrathin glomerular membranes. Q J Med 1995; 88: 785-793.

22. Flinter $F_{.4}$ Cameron $J .$, Chantler C., Houston I., Bobrow M. Genetics of classical Alport's syndrome. Lancet 1988; ii: 1005-1007.

23. Mochizuki T., Lemmink H., Mariyama M., Antignac C., Gubler A., Pirson Y., Verellen-Dumoulin C., Chan B., Schröder C., Smeets H., Reeders S. Identification of mutations in the $\alpha 3(\mathrm{TV})$ and $\alpha 4(\mathrm{TV})$ collagen genes in autosomal recessive Alport syndrome. Nat Genet 1994; 8: 77-82. 
73 


\section{Chapter 4.}

Chronicity index and mesangial IgG deposition are risk factors for hypertension and renal failure in early IgA nephropathy

Results of a long-term prospective epidemiological study 


\section{Summary}

In order to determine the natural history in early IgA nephropathy we evaluated the long-term follow-up of 27 nomotensive non-azotemic adult idiopathic IgA nephropathy patients with chronic haematuria who derived from a prospective regional epidemiological study of glomerulonephritis conducted between 1978-1984 in The Netherlands. As controls 17 thin GBM patients, 24 patients with normal renal tissue and 9 patients with miscellaneous nephropathies were followed. Median follow-up was 11 years, range 8-14 years. Renal biopsies were scored semi-quantitatively in terms of activity and chronicity indices, using a modified NIH scoring system for lupus nephritis.

During follow-up 2 patients with IgA nephropathy went into histological remission, 4 IgA nephropathy patients developed isolated hypertension, 3 patients developed isolated increased proteinuria, 2 patient developed hypertension in combination with proteinura and 3 patients developed renal failure, necessitating dialysis in one. Initial proteinuria over 1 gram/day was associated with a high activity score, extracapillary lesions and with late onset of uraemia. Mesangial IgG deposition and a higher initial chronicity index were associated with development of hypertension during follow-up. Circulating immune complexes, IgG/IgA theumatoid factor, age and gender had no bearing on disease history. In the multivariate analysis, a high initial chronicity index, erythrocyturia and mesangial IgG deposition are independent determinants of natural history of disease.

We conclude that in patients with IgA nephropathy, identified early in the course of disease, erythrocyturia, a high chronicity index and mesangial IgG deposition in the presence of normal renal function are risk factors for decreased renal survival. Disappearance of haematuria is associated with remission of $\operatorname{IgA}$ nephropathy immunopathologically and a low activity and chronicity index at initial biopsy. 


\section{Introduction}

Primary IgA nephropathy is the most prevalent nephrological cause of chronic macroscopic or microscopic haematuria in adults between 16-65 years of age [1]. Roughly one third of the patients will eventually develop renal insufficiency [2]. The pathogenesis of this disease is still topic of debate with advocates of a local immune complex formation of $\operatorname{IgA} 1$, and occasionally IgA2 antibodies, to mesangial or trapped antigens, and others proposing selective trapping of circulating IgA immune complexes by Fc $\alpha$ receptors on mesangial cells [3] or other mesangial receptors [4]. It is clear that these patients have an aberrant immune response, since immunisation with influenza-virus results in $\operatorname{IgA}$ and not in $\operatorname{IgM}$ and $\operatorname{IgG}$ antibodies [5]. Interestingly, when IgA nephropathy patients are immunised with a novel (cholera toxin subunit B) antigen intranasally, local and systemic IgA1-production appeared to be decreased in comparison to normal controls, suggesting a hyporesponsiveness of the mucosaassociated lymphoid tissue [6]. Many studies have tried to identify patients who are at risk for developing renal insufficiency and thus eligible for treatment. All published studies, however, have looked at a randomly selected population of IgA nephropathy patients, thus inevitably obscuring the natural history of $\operatorname{IgA}$ nephropathy as patients with long-standing disease are compared with patients with early renal disorder. Thus, signs of progression of renal disease may be confounded with risk factors for renal insufficiency in early disease. We conducted a prospective epidemiological study in which non-azotaemic normotensive IgA nephropathy patients were included at an early point in their natural history, comparing them to patients with thin GBM nephropathy, normal renal tissue and miscellaneous nephropathies that were biopsied for the same indication as the IgA nephropathy patients.

\section{Patients}

All patients were derived from a prospective epidemiological study conducted in three hospitals between 1978-1984 in the Netherlands. Briefly, in this study the incidence of idiopathic glomerulonephritis was determined by renal biopsy according to strict indications. Indications for renal biopsy (in hierarchical order as mentioned) were: 1 . recent onset uraemia (serum creatinine concentration $>300 \mu \mathrm{mol} / 1$ ), 2 . recent onset nephrotic syndrome, 3. persistent microscopic or recurrent macroscopic haematuria, 4. chronic proteinuria [1]. 
For a patient to be included in the present analysis, chronic haematuria had to be present for more than six months, but - to ensure evaluation of patients early in the natural history of disease - renal biopsy had to be performed within 2 years after patient identification. Urological examination, including intravenous urography, ultrasonography, cystoscopy and - occasionally - renal arteriography had to be negative. None of the patients were biopsied during bouts of macroscopic haematuria; at least six weeks passed between the last bout of macroscopic haematuria and the renal biopsy. Patients with known systemic disease, liver disorders, chronic hypertension or from famillies with known hereditary renal disease were excluded from analysis.

All idiopathic $\operatorname{IgA}$ nephropathy (IgAN) patients met the following criteria: (1) predominant deposition of $\operatorname{Ig} A_{1}$ and $C_{3}$ mesangially (with variable deposits of $\operatorname{Ig} A_{2}$, IgG and IgM) (2) mesangial electron-dense deposits on ultrastructural analysis and ( 3 ) no signs or symptoms of secondary IgA deposition, e.g. liver disease, ankylosing spondylitis or Henoch Schönlein nephritis. Thirty-one IgAN patients were identified, all presenting with chronic haematuria, two of which had an increased serum creatinine concentration (creatinine $>120 \mu \mathrm{mol} / \mathrm{l}$, but $<140 \mu \mathrm{mol} / \mathrm{l}$ ) at the time of renal biopsy. Of the 29 non-azotaemic patients, one patient was lost during follow-up and one patient developed severe rheumatoid arthritis during follow-up; hence they are excluded from the analysis of natural history. As epidemiological controls for the 27 non-azotaemic IgA nephropathy patients 17 patients with thin GBM nephropathy, 24 patients with normal renal tissue and 9 patients with miscellaneous nephropathies ( $3 x$ interstitial nephritis, $2 \mathrm{x}$ focal segmental mesangioproliferative glomerulonephritis, $2 \mathrm{x}$ focal global glomerulosclerosis, $1 x$ focal and segmental glomerulosclerosis, $1 x$ diffuse intracapillary glomenlonephritis) were followed as well. These controls derived from the same prospective study and were biopsied for the same indication (chronic persistent erythrocyturia on recurrent haematuria of six months duration, biopsied within two years of presentation) as the $\lg A$ nephropathy patients [7]. Follow-up was 11 years median, range $8-14$ years. Follow-up consisted of clinical examination, senum and 24-hour urine collection. Disease progression was clinically defined as development of renal impairment (serum creatinine concentration $>140 \mu \mathrm{mol} / \mathrm{i}$ in the absence of GFR-reducing therapy), development of bypertension during follow-up $(>140 / 90 \mathrm{~mm} \mathrm{Hg}$ ), increased proteinuria (at least twice the initial value, with proteinuria at follow-up over 0.5 gram/day, or persistently more than 1.0 gram/day) or a combination of these three. Six repeat renal biopsy were taken because of clinical progression of disease: development of hypertension (1x), impaired renal function 
$(2 x)$, increased proteinuria ( $3 x)$; another two patients underwent repeat renal biopsy because of disappearance of haematuria.

\section{Methods}

Renal biopsies were processed for light microscopy, immunofluorescence and ultrastructural analysis as described elsewhere [1]. Renal biopsies were classified according to the criteria as defined by the World Health Organisation [8], and had to contain more than five glomeruli per section to qualify for evaluation. At the time of renal biopsy routine laboratory tests included antinuclear and rheumatoid factor, antistreptolysin titre, classical hemolytic complement activity and liver function tests. Protein excretion was determined using the biuret method, adequate 24-hour urine collection was verified by measuring creatinine excretion

\section{Histologic scoring}

To assess the activity and chronicity of the lesions in IgA nephropathy serial 2 $\mu \mathrm{m}$ sections were stained with silver methenamin and counter stained with hematoxylin eosin. Of each biopsy serial sections containing the greatest numbers of glomeruli were used to determine the activity and chronicity index according to modified NIH criteria, originally developed for SLE [9], as follows. The activity index (AI) scored the presence of mesangial cell proliferation, segmental (noncircumferential) cellular crescent formation and mononuclear interstitial nephritis. Presence of mesangial cell proliferation and mononuclear interstitial nephritis was scored in a range of $0-3$, where 0 means absent, 1 means mild $(10-30 \%$ of all glomeruli involved, or focal interstitial infiltrate), 2 means moderate (30-70\% of all glomeruli involved, or multiple foci of interstitial infilitrate) and 3 means severe ( $>70$ $\%$ of all glomeruli involved or diffuse interstitial infiltrate). Presence of cellular crescents was scored in a range of $0-3$, where 0 means absent, 1 means less than $10 \%$ of the glomeruli involved, 2 means $10-30 \%$ glomeruli involved and 3 means more than $30 \%$ glomeruli involved. Cellular crescent scores were multiplied by 2 as their presence was considered to be more ominous; the maximal activity score amounted to 12. The chronicity index (CD) scoted the presence of global glomerulosclerosis, fibrous crescents, focal and segmental glomerulosclerosis, and interstitial fibrosis and tubular atrophy. Focal and segmental glomerulosclerosis was also weighed double; the maximal chronicity index amounted to 18. 
Table 1. Indication for renall biopsy, NIH indices and renal survival in 19 SLE patients from a prospective study on idiopathic glomerular disease carried out by the Regional Working Party on Renal Diseases in Limburg.

Indication

Number of patients

mean $\mathrm{Al} \pm \mathrm{sd}$

mean $\mathrm{CI} \pm \mathrm{sd}$

$* \mathrm{p}<0.05$
EP

$\begin{array}{lll}7 & 4 & 8 \\ 5.2 \pm 3.2 & 9.0 \pm 3.6^{*} & 9.3 \pm 7.9 \\ 1.3 \pm 2.0 & 1.8 \pm 1.7 & 1.1 \pm 1.58\end{array}$

\section{Validation of the NHH score as modified for IgA nephropathy}

Since the NIH score for SLE nephritis consists of an activity and chronicity index, we validated the modified NIH score for IgA nephropathy by comparing the lesions and activity index in renal biopsies of 19 untreated SLE nephritis, with the lesions seen in IgA nephropathy. The 19 SLE patients (16 female) were biopsied, within six months of developing symptoms of renal disease: erythrocyturia and proteinuria over 1 g/day $(7 \mathrm{x})$, recent onset nephrotic syndrome $(8 \mathrm{x})$ or erythrocyturia with proteinuria and uraemia (serum creatinine $120-300 \mu$ mol/1, $4 x$ ). As shown in Table 1. chronicity index was low in all groups, while an activiy index over 9 was associated with uraemia or nephrotic syndrome at biopsy. High activity indicated poor long term renal survival, regardless of therapy (azathioprine and steroids or cyclophosphamide and steroids) as eight year renal survival was $60 \%$ in biopsies with an activity score $<7$, while survival was $25 \%$ in biopsies with an activity $>7$. The NIH score was subsequently applied to score renal biopsies of 80 randomly biopsied IgA nephropathy patients (mean age $42 \pm 16$ years, mean estimated duration of disease $226 \pm 312$ weeks). Fibrinoid necrosis was rarely present in IgA nephropathy ( 2 of 80 patients, $2.5 \%)$ and when observed associated with widespread cellular crescents. Hyaline thrombi and wire loops (0 of 80 patients) and glomerular leucocyte infiltration ( 0 of 80 patients) were not found, hence these items were not incorporated in the modified NIH score for IgA nephropathy. Conversely, focal and segmental 
g]omerulosclerosis was present in 34 of 80 (43\%) randomly biopsied IgA nephropathy patients; thus we included this item in the novel, modified NIH scoring index (Table 2.). Using the modified $\mathrm{NHH}$ index, the 80 randomly biopsied patients had an activity index of $3.5 \pm 2.4$ (range 0 to 10 ) and a chronicity index of $6.2 \pm 4.4$ (range 0 to 15 ). In these IgAN patients, chronicity scores over 3 were associated with presence of mild to moderate azotaemia (serum creatinine 120 - $300 \mu \mathrm{mol} / 1$ ) at renal biopsy.

Table 2. Histological lesions observed in 80 randomly biopsied IgA nephropathy patients presenting with longstanding haematuria, scored according to the original NIH index as developed for SLE nephritis

$$
\text { present in renal biopsy }
$$

Activity scores:

- Mesangial cell proliferation

73

2

26

- Cellular crescents

0

0

51

- Interstitial mononucl. infiltrate

\section{Chronicity scores:}

- global glomerulosclerosis

44

64

- Interstitial fibrosis

- Tubular atrophy

67

- Focal segmental glomerulosclerosis

\section{Circulating immune complexes}

Immune-complexes were measured in a fluid phase $\mathrm{Clq}$ binding assay as described by Zubler et al. [10]. Resolubilised immune-complexes were determined in a solid phase anti-C3b-ELISA as described by Pereira et al. [11]. Extinctions of patient sera were related to a standard curve of heat aggregated human $\operatorname{IgG}$ and $\lg A$ 
immunoglobulins. Sera were considered positive when binding exceeded the 95 th percentile of nomal healthy controls; for IgG anti-C3b this was the equivalent of $6 \mu \mathrm{g}$ heat aggregated $\operatorname{IgG}$, and for $\operatorname{IgA}$ anti-C3b, the equiwalient of $3 \mu \mathrm{g}$ heat aggregated IgA. IgM anti-C3b immune-complexes were expressed as a percentage of binding compared with a pooled normal human serum: less than twice the extinction of normal serum was considered negative.

\section{$\operatorname{IgM} / \operatorname{Ig} A$ anti-IgG rheumatoid factor activity}

IgM and IgA rheumatoid activity was determined in an assay (modified from Otten et al. [12]) in which human IgG is coated by desiccation ovemight on PVC microtitre plates (ICN Pharmaceuticals, Zoetermeer, The Netherlands) in $0,01 \mathrm{M}$ sodium carbonate-buffer $\mathrm{pH}$ 9,6. After pre-incubation with phosphate-buffered saline containing $0,5 \mathrm{v} \%$ Tween-20 (PBS-Tween-20 0,5\%) for 30 minutes at room temperature, serial dilutions of patient sera were incubated at $50 \mu$ /well for 2 hours at $37^{\circ} \mathrm{C}$. After incubation the plates were washed and incubated with appropriate dilutions of goat $F(a b)_{2}$ anti-human IgM*HRP or goat $F(a b)_{2}$ anti-human IgA*HRP (Organon Teknica Cappel, Boxtel, The Netherlands) for 60 minutes at $37^{\circ} \mathrm{C}$. After incubation the conjugate was discarded, and bound antibody was detected by incubation with $5 \mathrm{mg}$ ortho-phenyl-diamine (OPD) in $10 \mathrm{ml}$ citrate-phosphate-buffer with $10 \mu \mathrm{I} \mathrm{H} \mathrm{H}_{2} \mathrm{O}_{2} 30 \%$. The reaction was stopped after 15 minutes by addition of $4 \mathrm{~N}$ $\mathrm{H}_{2} \mathrm{SO}_{4}$ and extinctions were read at an absorbency of $492 \mathrm{~nm}$. In each assay a negative and two positive control sera (one patient with known IgM anti-IgG, one with IgA anti-IgG) were included. Serum samples were considered positive when the optical density was twofold higher than that of a pool of normal healthy controls in at least three dillutions.

\section{$\operatorname{IgG}$ anti-IgA rheumatoid factor}

In this assay, human myeloma-derived $\lg \mathrm{A}_{1}$ was coated on PVC microtitre plates, while the assay was performed as described above. Presence of $\operatorname{IgG}$ in the coating material was excluded by using a patient serum with known IgM anti-IgG activity, which was negative on the IgA 1 -coating.

\section{Statistical analyses}

Statistical analyses were executed on a SPSS 4.0 package for Windows. In the univariate analysis, comparison between groups were done using the Mann-Whitney$U$ test for means and Fisher's exact test for actual counts. Kaplan Meier curves were 
drawn to evaluate the influence of several variables on the clinical disease-free interval. As endpoints for the Kaplan Meier curves all signs of disease-progression were considered (development of isolated hypertension, isolated increased proteinuria, development of uraemia, and the combination of development of hypertension with increased proteinuria). The tested variables were derived from previously published literature, where they were identified to play a role in the prognosis of IgA nephropathy. They included initial degree of proteinuria, gender, mesangial immune globulin depasition, initial type of haematuria (macroscopic versus microscopic), activity index and chronicity index.

\section{Results}

\section{Activity and chronicity index in $\operatorname{IgA}$ mephropathy}

In the prospective epidemiological study 29 patients (mean estimated duration of disease $55 \pm 58$ weeks) presented with non-azotaemic normotensive haematuria, 2 patients presented with haematuria and an increased serum creatinine concentration $(120-140 \mu \mathrm{mol} / 1)$. Renal biopsy in the latter two patients revealed lesions of chronic renal disease resulting in a high chronicity index $(10.5 \pm 2.1$; Table 3 ); follow-up revealed that both patients were dialysis-dependent within 5 years after renal biopsy. The mean chronicity index in IgA nephropathy patients with normal renal function was significantly lower $(2.4 \pm 2.3 ; \mathrm{p}=0.018)$.

When repeat renal biopsies were taken from six patients who had originally presented with normal renal function, but who had demonstrated signs of progressive renal disease during follow-up (indication for repeat renal biopsy: impained renal function $2 x$, hypertension $1 x$ and increased proteinuria $3 x$ ), an increase in chronicity score was seen (mean chronicity score at first biopsy $3.2 \pm 1.6$; at: second biopsy $6.2 \pm 2.7 ; p=0.059)$. The activity index of the repeat biopsy was not significantly different from the initial biopsy (the activity score was $1.3 \pm 1.5$ at first biopsy and $2.7 \pm 2.2$ at repeat biopsy; $\mathrm{p}=0.25$ ). The chronicity and activity indices in these six patients, who had a documented disease history of 11 years median, were comparable with those of the 80 randomly chosen $\operatorname{IgA}$ nephropathy patients (chronicity index $6.2 \pm 2.7$ in the repeat biopsies versus $6.2 \pm 4.4$ in the random biopsies; activity index in the repeat biopsies $2.7 \pm 2.2$ versus $3.5 \pm 2.4$ in the random ones). 
Table 3. Activity and chronicity indices in $29 \mathrm{IgA}$ nephropathy patients who were derived from the prospective study, as determined using the modified NIH score.

$\begin{array}{lll}\begin{array}{l}\text { Indication for biopsy in the } \\ \text { prospective stndy }\end{array} & \begin{array}{l}\text { mean actiwity } \\ \text { index } \\ \text { (range) }\end{array} & \begin{array}{l}\text { mean chronicity } \\ \text { index } \\ \text { (range) }\end{array} \\ \mathrm{E} / \mathrm{P}(\mathrm{n}=27) & \begin{array}{l}1.76 \pm 1.3 \\ (0-5)\end{array} & \begin{array}{l}2.38 \pm 2.31 \\ (0-8)\end{array} \\ \mathrm{E} / \mathrm{P} / \mathrm{U}(\mathrm{n}=2) & 2 & \\ & (2) & 10.5 \pm 2.12^{*} \\ & & (9-12)\end{array}$

Legend to the table

$\mathrm{E} / \mathrm{P}$ stands for erythrocyturia and proteinuria, E/P/U for erythrocyturia, proteinuria and uraemia (serum creatinine concentration $>140 \mu \mathrm{mol} / 1$ )

${ }^{*} \mathrm{p}=0.018 \mathrm{Mann}$-Whitney U-test

\section{Natural history: risk factors for progression of disease}

Hypertension developed in all patient groups that were studied. The incidence of hypertension was highest in miscellaneous group ( $44 \%, 4$ out of 9 patients), followed by thin GBM nephropathy ( $41 \%, 7$ out of 17 patients), and IgA nephropathy ( $20 \%, 6$ out of 27 patients). Two patients in the normal renal tissue group developed hypertension in the context of a positive familly history for bypertension (Table 4). In IgA nepluropathy mesangial IgG deposition in the initial biopsy was related to an increased risk for development of hypertension since 6 out of 8 patients who had IgG deposition initially, became hypertensive during follow-up, ( $p=0.006$ : Fisher's exact test). Development of hypertension did not coincide with the initial degree of proteinuria nor with the degree of proteinuria during follow-up. The initial chronicity score was associated with development of hypertension, as the mean $\mathrm{CI}$ score was $4.4 \pm 2.4$ in those patients who later developed hypertension, while it was $2.0 \pm 2.1$ in those who remained normotensive, (Mann-Whitney U-test: $p=0.018$ ). 
Table 4. Follow-up findings in patients with chronic haematuria

Immunopathological diagnosis

IgAN Thin GBM normal renal miscellaneous ${ }^{2}$ nephropathy tissue

Initial presentation:

Number of patients

Male:female

Mean age (years)

Macroscopic haematuria

Microscopic haematuria

$\begin{array}{llll}27^{1} & 17 & 24 & 9 \\ 20: 7 & 9: 8 & 21: 3 & 4: 5 \\ 31 & 35 & 32 & 38 \\ 10 & 1 & 12 & 2 \\ 17 & 16 & 12 & 7 \\ & & & \\ 7 & 10 & 17 & 3 \\ 5 & 5 & 3 & 2 \\ 3 & 1 & 3 & - \\ 12 & 1 & 1 & 4\end{array}$

Follow-up 11 years

Remission of haematuria

Hypertension

Increase in proteinuria

Uraemia

$\begin{array}{llll}2 & 0 & 17 & 3 \\ 8 & 7 & 2 & 4 \\ 3 & 0 & 0 & 0 \\ 3 & 0 & 0 & 1\end{array}$

Legend to the table

${ }^{1}$ Of the 31 idiopathic IgA nephropathy patients who were biopsied in the prospective epidemiological study, one was lost to follow-up, one developed severe rheumatoid arthritis and two patients with increased serum creatinine at the time of renal biopsy were excluded from the analysis of natural history.

2 interstitial nephritis $(x 3)$, glomerulonephritis $(x 3)$, focal global glomerulosclerosis $(\mathrm{x} 2)$, focal segmental glomerulosclerosis (x1)

Uraemia was found in the patients with IgA nephropathy ( $3 x$ ) and the miscellaneous group (1x), but not in the thin GBM or normal renal tissue group (Table 4). In IgA 
nephropathy the severity of the initial proteinuria was a risk factor for the development of uraemia and hypertension associated with increased proteinuria (Table 5). Initial proteinuria was associated with higher initial activity score $(p=0.008)$. Extracapillary lesions in IgA nephropathy, such as small cellular crescents, fibrous crescents, synechiae and adhesions to the capsule of Bowman, were associated with a higher initial proteinuria (mean $1.3 \mathrm{~g} /$ day in extracapillary vs. $0.5 \mathrm{~g} / \mathrm{day}$ in non-extracapillary glomerulonephritis; $\mathrm{p}=0.004$ ) and with a higher activity and chronicity score (AI in extracapillary $\operatorname{IgAN} 3.1 \pm 2.1$, AI in normextracapillary $\operatorname{IgAN} 1.4 \pm 1.0 ; \mathrm{p}=0.02 ; \mathrm{CI}$ in

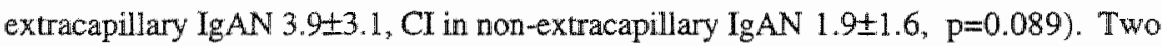
patients with crescentic glomerulonephritis developed impaired renal function, while one patient with non-crescentic glomerulonephritis - but with proteinuria $>1 \mathrm{~g} /$ day had an increased serum creatinine concentration at follow-up. Crescentic disease did not occur in those patients $(n=6)$ who had no mesangial proliferation in the initial biopsy and no renal failure developed in these patients. Two patients who had extracapillary lesions in more than $30 \%$ of the glomeruli, had the poorest prognosis, for it was associated with renal function deterioration in both (data not shown).

Table 5. Effect of proteinuria on the natural history of IgA nephropathy. Patients were normotensive and non-azotaemic nt the time of renal biopsy

\begin{tabular}{lcccccc} 
Proteinuria & no. & median & \multicolumn{3}{l}{ End follow-up } \\
& & follow-up & H+P & U & $p$ \\
\hline$<1000 \mathrm{mg} / 24 \mathrm{~h}$ & 15 & $\mathbb{2}$ & 0 & 0 & \\
$>1000 \mathrm{mg} / 24 \mathrm{~h}$ & 12 & 11 & 2 & 3 & $<0.00981$
\end{tabular}

legend to the table

$\mathrm{H}=$ hypertension $(\mathrm{RR}>140 / 90 \mathrm{~mm} / \mathrm{Hg}) ; \mathrm{P}=$ increase in proteinuria; U =uraemia (serum creatinine concentration $>140 \mu \mathrm{mol} / \mathrm{l}$ )

1 Fisher's exact test 


\section{Univariate versus multivariate analysis}

In the univariate analysis development of hypertension is associated with mesangial deposition of IgG and a higher chronicity index, while development of uraemia is associated with initial proteinuria over $1000 \mathrm{mg} / \mathrm{day}$. High initial proteinuria is related to a high activity index and to extracapillary crescents, but not to development of hypertension, as illustrated above. Kaplan Meier curves showed that mesangiall IgG deposition, initial type of haematuria and a chronicity index higher than 3 had a significant influence on the clinical disease-free interval, as illustrated in Figure $1 \mathrm{a}, \mathrm{b}, \mathrm{c}$. When the same analysis was performed using the clinically more severe endpoints, like development of uraemia and development of proteinuria in combination with hypertension, inital proteinuria over $1000 \mathrm{mg} / \mathrm{day}(\mathrm{p}=0.02)$, erythrocyturia as presenting symptom ( $\mathrm{p}=0.032)$ and an initial chronicity index over $3(\mathrm{p}=0.0247)$ were related to disease progression. Cox regression showed that, of the tested variables, a chronicity index higher than 3, initial type of haematuria and mesangial IgG deposition were independent determinants of renal survival.

\section{Repeat renal biopsies}

Six patients underwent repeat renal biopsies because of clinical progression of disease: the indications were development of non-proteinuric hypertension (1x), increased proteinuria $(3 \mathrm{x})$ and development of impaired renal function $(2 \mathrm{x})$. Two patients underwent repeat renal biopsy because of disappearance of haematuria. Histologically, the latter biopsies showed normal renal morphology, disappearance of IgA and complement C3 deposition and the previously present mesangial clense deposits were no longer visible ultrastructurally. Two of three repeat biopsies taken because of increased proteinuria, showed a striking increase in mesangial IgG deposition, that initially had only been trace positive. The two repeat biopsies, taken because of mildly impaired renal function, showed diffuse mesangial cell proliferation with signs of chronic renal disease. In one patient this was combined with cellular extracapillary crescents. The remaining two patients showed ongoing disease without changes in activity or in chronicity.

\section{Circulating immune complexes and rheumatoid factors}

We investigated whether the presence of circulating immune-complexes and theurnatoid factor was related to the natural history of $\operatorname{IgA}$ nephropathy by testing 27 IgA nephropathy patient sera and comparing them to the control group. The results are summarised in Table 6. Circulating immune-complexes of the IgA subclass were 
detected in four IgA nephropathy patients at the time of renal biopsy, but in thin GBM nephropathy and normal renal tissue respectively two and four patients also had circulating IgA immune-complexes [13]. IgA rheumatoid factor was only found in 3 patients in the IgA nephropathy group. After 11 years follow-up two IgA nephropathy patients denonstrated IgA-containing inmune-complexes. IgA rheumatoid factor was no longer demonstrable in the IgA nephropathy group at follow-up. Circulating immune-complexes as measured in the Clq binding assay and anti-C3b assay were not related to the natural history of IgA nephropathy. Moreover, in thin GBM nephropathy and in patients with normal renal tissue only low titre circulating immune-complexes were found.

As mesangial $\mathrm{IgG}$ deposition appeared to be associated with progressive renal disease in IgAN we tested whether IgG deposition in the kidneys could be explained by IgG anti-IgA rheumatoid factor activity. No rheumatoid factor activity could be found in the sera of these patients against purified human $\operatorname{IgA}$.

Legend to figure 1

Survival curves for mesangial Ig deposition (figure 1a), chronicity index (figure 1b) and initial type of haematuria (figure 1c). All signs of disease-progression are considered: development of hypertension, increased proteinuria, development of uraemia and a combination of hypertension and increased proteinuria. Mesangial IgG deposition $(p=0.0085)$ and an initial chronicity index above $3 \quad(p=0.029)$ were associated with a decreased clinical disease-free interval, while erythrocyturia as presenting symptom just failed to achieve statistical significance $(p=0.062)$ 
Figure 1. Survival curves in IgA nephropathy

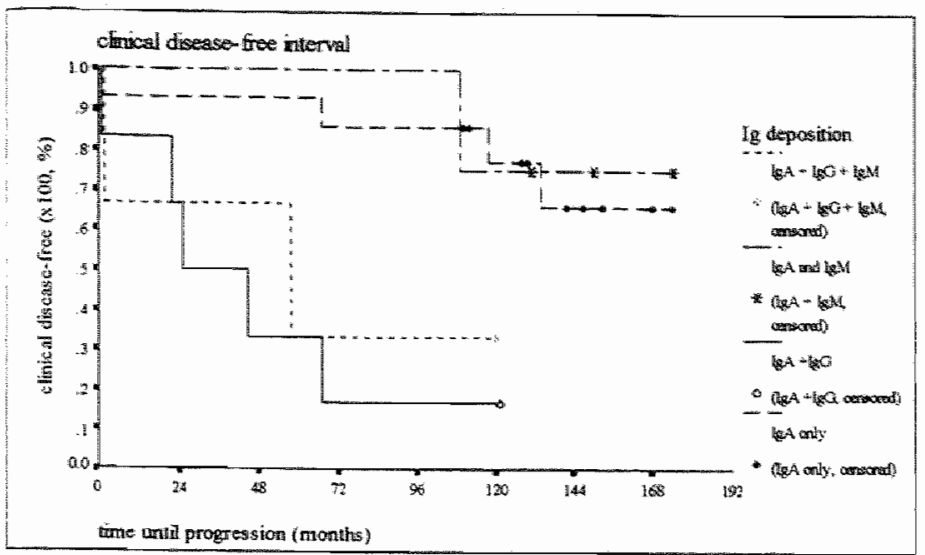

figure la.

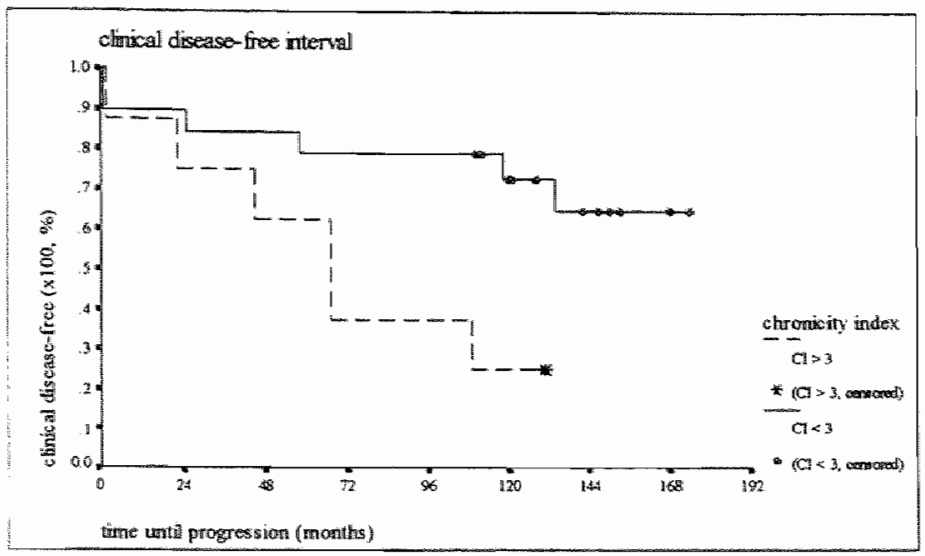

figure $1 \mathrm{~b}$.

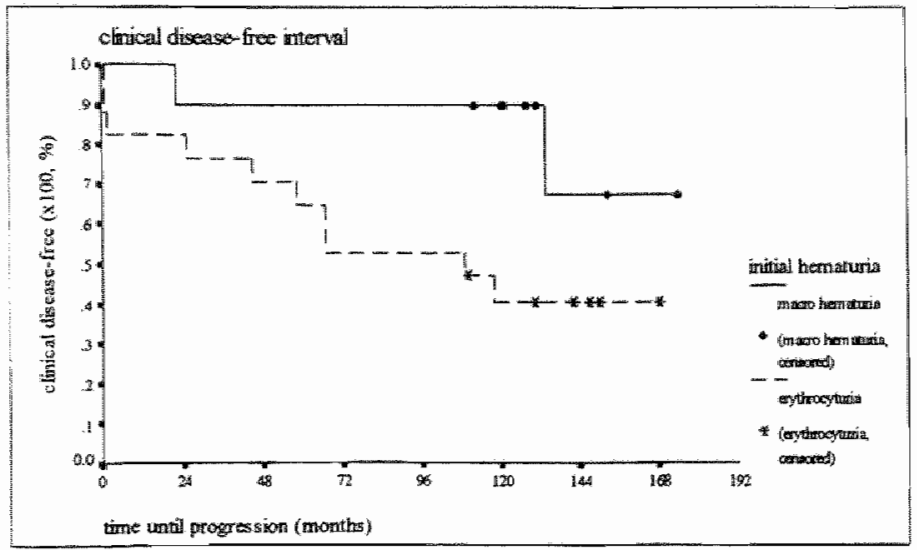

figure $1 \mathrm{c}$. 
Table 6. Circulating immune complexes in idiopathic chronic haematuria as determined at the time of renal biopsy and at 11 years follow up in idiopathic haematuria.

\begin{tabular}{lll}
$\lg A N$ & Thin GBM & normal \\
$(n=27)$ & $(n=17)$ & $(n=24)$ \\
\hline
\end{tabular}

\section{At renal bionsy}

Clq binding assay Anti-C3b assay $\lg A_{1}$ Anti-C3b assay IgA2 Anti-C3b assay IgG Anti-C3b assay IgM

IgA rheumatoid factor

$\begin{array}{lll}0 & 0 & 0 \\ 4 & 1 & 4 \\ 0 & 1 & 0 \\ 0 & 0 & 0 \\ 0 & 0 & 0\end{array}$

At follow-up (11 years median)

Clq binding assay

$\begin{array}{lll}8 & 5 & 3 \\ 2 & 0 & 0 \\ 1 & 2 & 1 \\ 5 & 1 & 4\end{array}$

IgA rheumatoid factor

0

0

3

0

1

4

Anti-C3b assay IgM

0

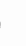

\section{Discussion}

Reviewing 34 studies published prior to 1985 encompassing 2317 patients with $\operatorname{Ig}$ A nephropathy, Emancipator et al. concluded that in the vast majority of patients the disease had an indolent course with $20-50 \%$ of the patients developing end-stage renal disease in a 20 year period $[2,14]$. Other retrospective studies from Italy [15] and the UK [16], each encompassing over 600 patients, have confirmed the slowly progressive course of the disease with a 10 year actuarial renal survival from the time of renal biopsy of $83 \%$ [16] and a 20 year actuarial renal survival of $75 \%$ [15], when taken from the estimated onset of disease. Renal biopsies that were taken during bouts 
of macroscopic haematuria associated with azotaemia may show focal crescentic glomerulonephritis [17], the azotaemia in these cases is usually transient in nature and is thought to be brought about, at least partially, by erythrocyte casts causing tubular obstruction [18]. Crescentic lesions were not observed when renal biopsies were taken a few weeks after bouts of macroscopic haematuria [19]. A truly rapidly progressive crescentic form of IgA nephropathy does exist, however, but was only seen in 3 out of 374 patients and associated with glomerular crescents in over $50 \%$ of the glomeruli in two patients [15].

Previous studies have identified heavy microscopic haematuria $[2,19-21]$, the absence of gross haematuria $[15,19]$, the severity of proteinuria $[2,15,16,19,22]$, decreased renal function at the time of renal biopsy $[15,19]$ and uncontrolled hypertension [2, 15, 19] as risk factors for an unfavourable renal prognosis. Recently, several reports discussed ACE-gene polymorphisms as risk factors for progression in IgA nephropathy [23-26] When results of follow-up are rellated to the initial biopsy findings there is consensus that glomerular fibrosis of the global and segmental variety, interstitial fibrosis with interstitial infiltrates and vascular fibrosis and hyalinosis are associated with azotaemia, severe proteinuria and hypertension and hence with an unfavourable prognosis $[2,15,19,22,27,28]$. Whether or not the presence of focal and glomerular crescents is indicative of a poor prognosis is topic of debate: in several studies it is identified a risk factor $[17,20,21,29-33]$ in contrast to others $[19,28]$. A major drawback of these studies is that patients identified early in the natural history of disease are inevitably mixed with those well on the way to endstage renal disease. Hence many of the risk factors, e.g. old age, hypertension, azotaemia and severe proteinuria are related [15], since they are secondary to a slowly decreasing renal mass, histologically characterised by global glomerular fibrosis, focal and segmental glomenulosclerosis, interstitial fibrosis and inflammation and vascular hyalinosis $[15,34]$.

The current prospective epidemiological study differs from previous studies in two major aspects. First, during the study period renal biopsies were taken from all patients with chronic idiopathic non-hypertensive haematuria of non-urological origin within 2 years after first presentation of symptoms. The current organization of the health care in the Netherlands, including urine analysis for insurance, sports and draft registration, enables asymptomatic patients to be identified early in the course of disease. Second, in order to verify that renal biopsies were taken early in the natural history of disease we determined the activity and chronicity indices using a modified NIH score system. Renal biopsies from 80 non-selected patients with erythrocyturia 
and proteinuria with or without mild renal impairment, showed high chronicity and - to a lesser extent - high activity indices. Our patients with primary IgA nephropathy and with a normal serum creatinine concentration were biopsied early in the natural history, as they had low chronicity and activity indices. In repeat renal biopsies, taken after a documented disease history of 11 year median because of development of hypertension, increase in proteinuria or decrease in creatinine clearance, high chronicity and activity indices were present. In our opinion, in patients with bigh activity scores, normal renal function and normal blood pressure, one might consider immunomodulating therapy [35-37], while high chronicity scores usually indicate long-standing disease, not eligible for immunotherapy. Interestingly, in a recent report on immunoglobulin therapy in $\operatorname{Ig} A$ nephropathy a high activity score, as scored by mesangial and epithelial cell proliferation, was reversed by treatment with parental immunoglobulins, at the cost of an increased histological sclerosis index [38].

The development of hypertension during the natural history of IgA nephropathy has been reported to be an independent risk factor for renal insufficiency [39], while lesions of hypertension have been used in scoring $\operatorname{IgA}$ nephropathy $[20,21,27-29$, $33,40-42$ ]. We have not included vascular lesions in our index, since none of the patients included in our study had hypertension at renal biopsy. No lesions attributable to hypertension were seen in these initial biopsies. The incidence of renal hypertension after 11 year follow-up is identical in patients with non-azotaemic IgA nephropathy and with thin GBM nephropathy. Renal function studies in thin GBM nephropathy indicate that increased loss of renal mass due to the original disease precedes the hypertension [43], casting doubt on the independency of the hypertension in terms of causing renal insufficiency. Mesangial deposition of $\mathrm{IgG}$ was associated with development of hypertension. This could be related to the greater potential of $\operatorname{IgG}$ to initiate and sustain an inflammatory process, thus interfering with the paracrine and endocrine functions of the mesangium. IgA itself does not activate classical complement. Whether mesangial $\operatorname{IgG}$ is directed to the same antigens as the $\operatorname{IgA}$ deposition, to mesangial cell constituents [44-46] or due to rheumatoid factor activity to deposited IgA is unclear. The latter alternative is unlikely, as $\operatorname{IgG}$ anti-IgA rheumatoid activity was absent in sera obtained at the time of initial biopsy and at follow-up (unpublished observation). Moreover, no Clq deposition was demonstrable in the mesangial immune complexes, although ex vivo generated $\operatorname{IgG}$ anti $\operatorname{IgA}$ rheumatoid factors readily bind $\mathrm{Clq}$. However, glomerular IgG rheumatoid factor activity can not be ruled out as elution studies of glomerular deposited IgG were not done. 
In the multivariate analysis we showed that an increased chronicity index, mesangial IgG deposition and erythrocyturia as presenting symptom are independent determinants of renal survival in early IgA nephropathy. Increased proteinuria during the follow-up period occurred in $11 \%$ of our patients, which is comparable to what was found in other studies [22]. Microscopic haematuria was associated with a poor prognosis, when compared with macroscopic haematuria. Nevertheless, two erythrocyturic patients with primary IgA nephropathy went into histological remission at cessation of haematuria, an observation which has previously only been described in children [47]. Others have reported heavy continuous microscopic haematuria, or absence of macroscopic bouts, to be a risk factor for renal insufficiency in $\operatorname{Ig} A$ nephropathy, but it is not clear whether this was due to patients being identified late in the natural history of disease. When the chronicity indices of our erythrocyturic IgA nephropathy patients are compared with those of the patients with the macroscopic variant of IgA nephropathy, the index in the former group was higher than in the latter. Although statistically not significant, this suggests that the microscopic variant is detected later in the natural history of disease and therefore might be associated with a relatively poor prognosis.

Crescentic IgA nephropathy has been considered a bad prognostic sign in many studies $[17,20,21,29-33]$. In this study, the presence of extracapillary lesions was associated with high initial proteinuria but not with renal insufficiency at follow-up. However, it seems reasonable to assume that the presence of glomerular cellular crescents, in the absence of recent macroscopic haematuria, early in the natural history of disease is a risk factor for progressive disease, as it indicates active inflammation of the glomerulus. Whether or not mesangial $\operatorname{lgG}\left(\mathrm{IgG}_{1}\right.$ and $\mathrm{IgG}_{3}$ ) deposition favours crescent formation is unclear, we did not observe a relation between mesangial $\mathrm{IgG}$ deposition and presence of crescents. 
Legend to figure 2

A. Immunofluorescence study of active primary IgA nephropathy. The mesangium stains diffusely positive for IgA and C3 (not shown) in a mesangial granular pattern, with occasional capillary deposition $(100 \mathrm{x})$

B. Immunofluorescence study of primary $\operatorname{IgA}$ nephropathy in remission. Clinically, erythrocyturia is no longer present, at immunohistochemical analysis only trace staining for IgA and negative staining for C3 is present (160x)

C. Ultrastructural analysis of active primary $\operatorname{Ig} A$ nephropathy. The mesangium shows. clear deposition of electron dense material, with sparing of the capillary loops (6660x)

D. Ultrastructural analysis of primary IgA nephropathy in remission. Increase in mesangial matrix with thickening of the capillary is visible, but no electron dense deposits are present in the mesangium $(6660 \mathrm{x})$

E. Primary IgA nephropathy with active focal segmental mesangioproliferative glomerulonephritis and small cellular extracapillary component (arrow). The interstitium shows a normal aspect (silver methenamin hematoxylin eosin stain, 65x.) F. Primary IgA nephropathy with chronic lesions like fibrous extracapillary crescent formation (arrow), tubule atrophy and interstitial inflammation and fibrosis (silver methenamin hematoxylin eosin stain, 65x.) 
Figure 2. Immunohistochemical and ultrastructural remission of IgA nephropathy
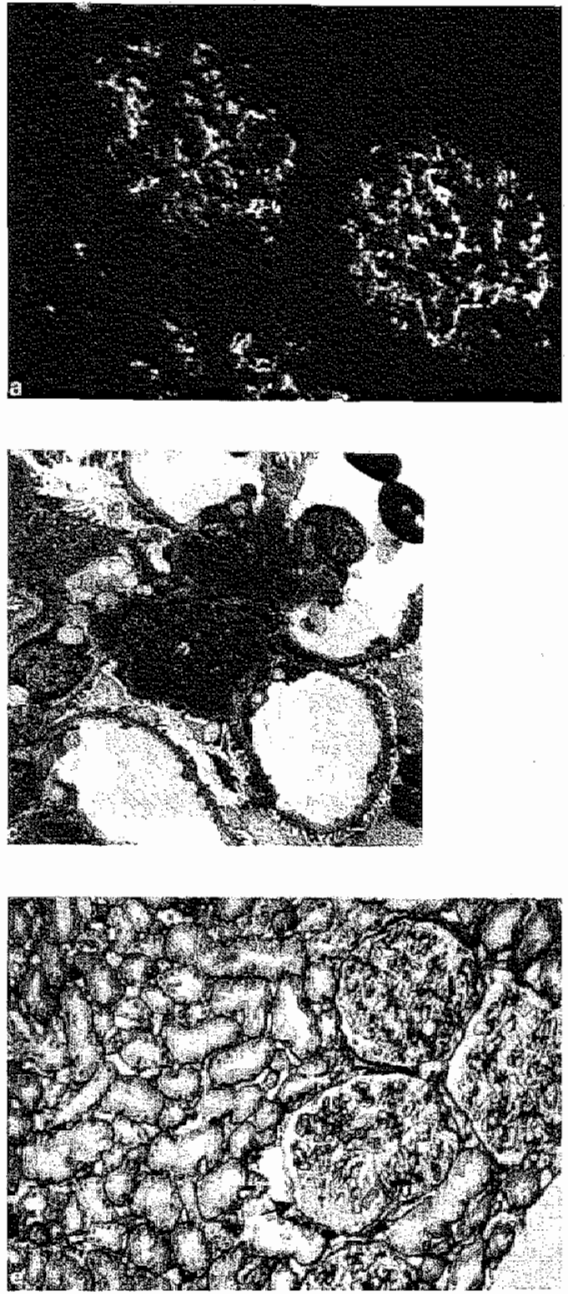
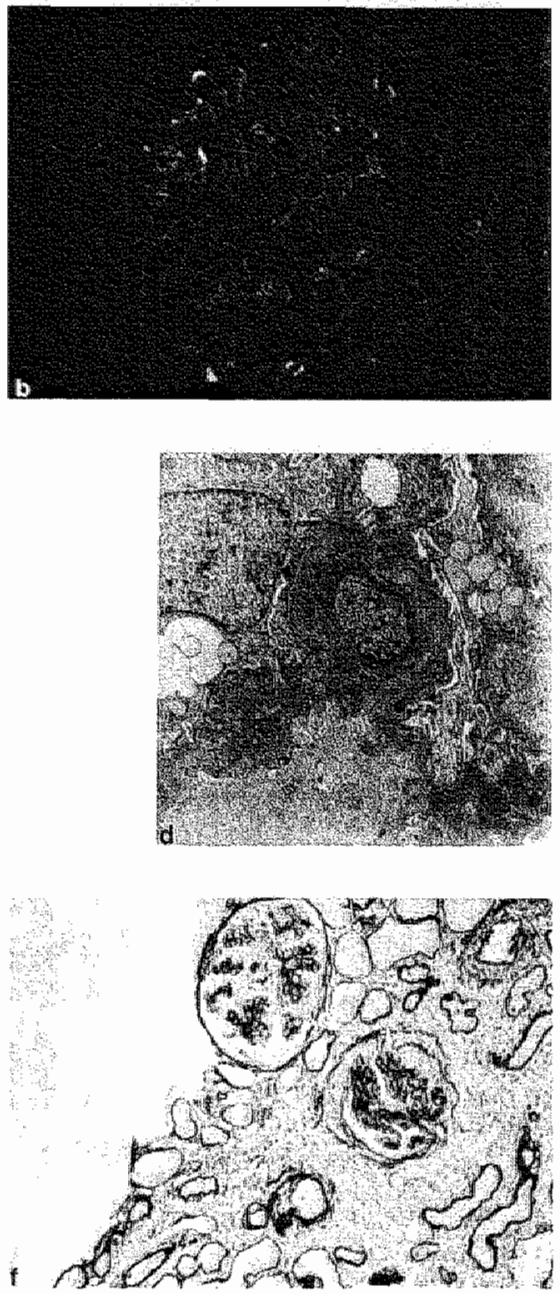


\section{References}

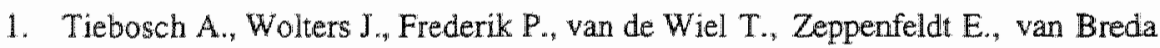
Vriesman P. Epidemiology of idiopathic glomerular disease: A prospective study. Kidney Int $1987 ; 32: 112-116$.

2. Emancipator S., Gallo GR., Lamm M. IgA nephropathy: perspectives on pathogenesis and classification. Clin Nephrol 1985;24:161-179.

3. Gomez-Guerrero $\mathbb{C}_{\text {, }}$ Gonzalez $\mathbb{E}$., Egido J. Exidence of a specific IgA receptor in rats and human mesangial cells. J Immunol 1993; 151: 7172-7181.

4. Lai K., To W., Li P., Leung J. Increased binding of polymeric lambda-IgA to cultured human mesangial cells in IgA nephropathy. Kidney Int 1996; 49: 839845 .

5. Van den Wall Bake A., Beyer W. Evers-Schouten J., Hermans J., Daha M., Masurel N., van Es L. Humoral immune response to influenza vaccination in patients with primary immunoglobulin A nephropathy. An analysis of isotype distribution and size of the influenza-specific antibodies. J Clin Invest 1989;84: 1070-1075.

6. de Fijter J., Eijgenraam J., Braam C., Holmgren J., Daha M., Van Es L., van de Wall Bake W. Deficient IgAl immune response to nasal cholera toxin subunit $B$ in primary IgA nephuropathy. Kidney Int 1996; 50: 952-961.

7. Nieuwhof C, Doorenbos C., Grave W., De Heer F., De lleeuw P., Zeppenfeldt E, Van Breda Vriesman P. A prospective study of the natural history of idiopathic non-proteinuric hematuria. Kidney Int 1996; 49:

8. Churg J., Bernstein J., Glassock R.: Renal Disease (second edition). Tokyow New York, Igaku-Shoin, 1995.

9. Austin H., Muenz L., Joyce K., Antonovych T., Kullich M., Klippel J., Decker J., Balow J. Prognostic factors in lupus nephritis, contribution of histological data. Am J Med 1983; 75: 382-391.

10. Zubler R., Lange G., Lambert P., Miescher P. Detection of immune complexes in unheated sera by a modified 125I-Clq binding assay. I Immunol 1976; 116: 232235.

11. Pereira A., Theophilopoulos A., Dixon F. Detection and partial characterization of circulating immune complexes with solid-phase anti-C3. J Immunol 1980; 125 : $763-770$. 
12. Otten H., Daha M., de Rooij H., Breedveld F. Quantitative detection of classspecific rheumatoid factors using mouse monoclonal antibodies and the biotin/streptavidin enhancement system. Brit J Rheumatol 1989; 28: 310-316.

13. Tiebosch $\mathrm{T}$. Idiopathic glomerular disease, a prospective regional study. Maastricht 1987.

14. Ibels L., Gyory A. IgA nephropathy: analysis of the natural history, important factors in the progression of renal disease and a review of the literature. Medicine 1994; 73: 79-102.

15. D"Amico G., Imbasciati E., Barbiano di Belgioioso G., Bertoli S., Fogazzi G., Ferrario F., Fellin G., Ragni A., Colasanti G., Minetti L., Ponticelli C. IgA nephropathy. Medicine $1985 ; 64: 49-60$.

16. Johnston P., Brown J., Braumholtz D., Davison A. Clinico-pathological correlations and long-term follow-up of 253 UK patients with IgA nephropathy. Q J Med 1992; 84: 619-627.

17. Bennett W., Kincaid-Smith P. Macroscopic Lematuria in mesangial IgA nephropathy: correlation with glomerular crescents and renal dysfunction. Kidney Int $1983 ; 23: 393-400$.

18. Delcaux $C_{\text {., Jacquot }} C_{\text {, }}$, Callard $P$., Kleinknecht $D$. Acute reversible renal failure with macroscopic haematuria in IgA nephropathy. Nephrol Dial Transplant 1993; 8: 195-199.

19. Beukhof J., Kardaun O., Schaafsma W., Poortema K., Donker A., Hoedemaeker P., van der Hem G. Towards individual prognosis of IgA nephropathy. Kidney Int $1986 ; 29: 549-556$.

20. Nicholls K., Fairley K., Dowling J., Kincaid-Smith P. The clinical course of mesangial IgA associated nephropathy in adults. Q J Med 1984; New Series LIIII: $227-250$.

21. D'Amico G., Minetti L., Ponticelli C., Fellin G., Ferrario F., Barbiano di Belgioioso G., Imbasciati E., Ragni A., Bertoli S., Fogazzi G., Duca G. Prognostic indicators in idiopathic IgA mesangial nephropathy. Q I Med 1986; New Series 59: $363-378$.

22. Neelakantappa K., Gallo GR., Baldwin DS. Proteinuria in IgA nephropathy. Kidney Int 1988; 33: 716-721.

23. Harden P., Geddes C., Rowe P., Mclioy J., Boullton-Jones M., Rodger R., Junor B., Briggs J., Connell I., Jardine A. Polymorphisms in angiotensinconverting-enzyme gene and progression of IgA nephropathy. Lancet 1995; 345: $1540-1542$. 
24. Yorioka T., Suehiro T., Yasuoka N., Hashimoto K., Kawada M. Polymorphism of the angiotensin converting enxyme gene and clinical aspects of IgA nephropathy. Clin Nephrol 1995; 44: 80-85.

25. Yoshida H., Mitarai T., Kawamura T., Kitajima T., Miyazaki X., Nagasawa R., Kawaguchi $Y .$, Kubo H., Ischikawa I, Sakai O. Rolle of the deletion polymorphism of the angiotensin converting enzyme gene in the progression and therapeutic responsiveness of IgA nephropathy. J Clin Invest 1995; 96: 21622169 .

26. Schmidt S., Stier E., Hartung R., Stein G., Bahnisch J., Woodroffe A., Clarkson A., Ponticelli C., Campise M., Mayer G., Ganten D., Ritz E. Na association of converting enzyme insertion/deletion polymorphism with immunoglobulin A glomerulonephritis. Am J Kid Dis 1995; 26: 727-731.

27. Alamartine E., Sabatin JC., Berthoux FC. Comparison of pathological lesions on repeated renal biopsies in 73 patients with primary IgA nephropathy: value of quantitative scoring and approach to final diagnosis. Clin Nephrol 1990; 34: 4551.

28. Okada H., Suzuki H., Konishi K., Sakaguchi H., Sanuti T. Histological alterations in renal specimens as indicators of prognosis of $\operatorname{IgA}$ nephropathy. Clin Nephrol 1992; 37: 235-238.

29. Clarkson A., Seymour A., Thompson AJ, Haynes W., Chan Y-L., Jackson B. IgA nephropathy: a syndrome of uniform morphology, diverse clinical features and uncertain prognosis. Clin Nephrol 1977; 8:459-471.

30. Lee $\mathbb{K}$., Rao V., Franklin W., Schiffer M., Aronson A., Spargo B., Katz A. IgAnephropathy: morphologic predictors of progressive renal disease. Hum Pathol $1982 ; 13: 314-322$.

31. Kobayashi Y., Tateno S., Hiki T., Shigematsu $\mathbb{H}$. IgA-nephropathy: prognostic significance of proteinuria and histologic alterations. Nephron $1983 ; 34: 146-153$.

32. Okada K., Funai K., Kagami S., Yano I., Kuroda Y. IgA nephropathy in Japanese children and adults: a comparitative study of climicopathological features. Am J Nephrol 1990; 10: 191-197.

33. Hotta O., Taguma Y., Kurosawa K., Sudo K., Suzuki K., Horigome I. Predictive value of small crescents in IgA nephropathy: analysis of four patients showing a deteriorated renal function during a long follow-up period. Clin Nephrol 1993; 40: $125-130$.

34. Widstam-Attorps U., Berg U., Bokman S., Lefvert A. Proteinuria and renal function in relation to renal morphology. Clin Nephrol 1992; 38: 245-253. 
35. Peh C., Clarkson A. Treatment of IgA nephropathy. Nephrol Dial Transplant 1995; 10: 1119-1121.

36. Goumenos D., Ahuja M., Shortland J., Brown C. Can immunosuppressive drugs slow the progression of IgA nephropathy? Nephrol Dial Transplant 1995; 10: 1173-1181.

37. Rostoker G., Deswaux- Belghiti D., Pilatte Y., Petit-Phar M., Philippon C., Deforges L., Terzidis H., Intrator L., André C., Adnot S., Bonin P., Bierling P., Remy P., Lagrue G., Lang P., Weil B. Immunomodulation with low-dose immumoglobulins for moderate IgA nephropathy and Henoch-Schónlein purpura. Nephron $1995 ; 69: 327-334$.

38. Rostoker G., Deswaux-Belghiti D., Pilatte Y., Petit-Phar M., Phillippon C., Deforges L., Terzidis H., Intrator I., Andre C., Lagnue G., Lang P., Weil B. High-dose immunoglobulin therapy for severe $\operatorname{IgA}$ nephropathy and Henoch Schönlein purpura. Ann Int Med 1994; 120: 476-484.

39. Rekola S., Bergstrand A., Bucht H. Development of hypertension in IgA nephropathy as a marker of a poor prognosis. Am J Nephrol 1990; 10: 290-295.

40. Hood S., Velosa J., Holley K., Donadio J. IgA-IgG nephropathy: predictive indices of progressive disease. Clin Nephrol 1981; 16:555-62.

41. Bogenschuitz O., Bohle A., Batz C., Wehrmann M., Pressler H., Kendziorra H., Gärtner H. IgA nephritis: on the importance of morphological and clinical parameters in the long-term prognosis of 293 patients. Am J Nephrol 1990; 10: $137-147$.

42. Radford M., Donadio J., Bergstrahl E., Grande J. Predicting renal outcome in IgA nephropathy. JASN 1997; 8: 199-207.

43. Nieuwhof C., De Heer F., de Leeuw P., van Breda Vriesman P. Thin GBM nephropathy: premature glomerular obsolescence is associated with hypertension and late onset renal failure. Kidney Int 1997; 51: 1596-1601.

44. Ballardie F., Brenchley P., Wiliams S., O'Donoghue D. Autoimmunity in IgA nephropathy. Lancet 1988 ; ii: 587.

45. ODonoghue D., Darvil V., Ballardie F. Mesangial cell autoantigens in immunoglobulin A nephropathy and Henoch Schoenlein purpura. J Clin Invest 1991; 88: 1522-1530.

46. Fornasieri A., Pinerolo C., Li M., Armelloni S., Gibelli A., D'Amico G. Antimesangial and anti-endothelial cell antibodies in IgA mesangial nephropathy. Clin Nephrol 1995; 44: 71-78. 
Chapter 4

47. Yoshikawa N., ljima K., Matsuyama S., Suzuki J., Kameda A., Osada S., Nakamura $H$. Repeat renal biopsies in children with IgA nephropathy. Clin Nephrol 1990; 33: 160-167. 
101 


\section{Chapter 5.}

Natural history of idiopathic membranous

glomerulonephritis

A prospective epidemiological study. 


\section{Summary}

A prospective regional epidemiological study was carried out over the period 1978-1988 in order to determine the incidence and natural history of idiopathic glomerular disease in adults between 16 and 65 years of age. For this study, renal biopsies were taken from all patients with signs or symptoms of idiopathic glomerular disease on the basis of strict indications early in the natural history of disease.

Of the renal biopsies, taken because of apparent recent onser nephrotic syndrome and chronic proteinuria, 25 had idiopathic membranous glomerulonephritis (iMGN). Twentyfour patients had a proteinuria over $5.0 \mathrm{~g} /$ day (or $>4.5 \mathrm{~g} / \mathrm{day}$ per $15 \mathrm{mmol} / \mathrm{l}$ urimary creatinine corrected for body surface area 1.73 m2) The patients were usually normotensive, all had a creatinine clearance $>60$ $\mathrm{ml} / \mathrm{min} / 1.73 \mathrm{~m} 2$ and stage 1 histology with glomerular $\mathrm{C} 3$ and $\mathrm{C} 5 \mathrm{~b}-9$ neoantigen deposition, in the absence of interstitial changes. One patient had chronic mild proteinuria and stage 3 histology. The incidence of iMGN was remarkably constant over the observation period and in the order of 10 per $10^{6}$ adults at risk/year. Twentyone patients, all presenting with recent onset nephrotic syndrome, were followed for 8 to 14 years ( 11 years median).

Of these patients, $80 \%$ was in complete $(57 \%)$ or partial $(23 \%)$ remission after 5 years, late recurrences of nephrotic syndrome occurred in $19 \%$ and chronic renal insufficiency developed, either very early or late, in 19\%. Thrombo-embolic phenomena occurred in $14 \%$, early after onset of nephrotic syndrome or after recurrence. Initial proteinuria had no bearing on prognosis. Chronic nephrotic syndrome was associated with an enhanced risk for chronic renal insufficiency. In the absence of early chronic renal failure however, a chionic nephrotic syndrome over 2 years duration carried a good prognosis with complete remission in $66 \%$. The chronic renal insufficiencies could be divided in two subsets: those which develop early in the disease history and are presumably immune-mediated and those which develop late and are not necessarily immune-mediated. Repeat renal biopsies are of required to differentiate one subset from the other. 


\section{Introduction}

The natural history of idiopathic membranous glomerulonephritis (MGN) has been described in retrospective [1,2] and prospective studies [3], each dealing with about 100 patients with follow ups of 5 years median. The common denominator in all of these studies is the scarcity of stage 1 lesions, and - the study of Noell et. al. [1] excepted - the absence of clinico-pathological correlations at inclusion. Thorough investigation of these studies shows that patients have been included on the basis of renal biopsy diagnosis, regardless of presenting sign or symptoms of disease, which included discrete proteinuria with normal renal function [3], uraemia, and nephrotic syndrome in the presence or absence of renal insufficiency $[1,2]$. These studies make an evaluation of risk factors for renal insufficiency such as age, degree of proteinuria and renal functions at inclusion, hazardous [4]. It has lately been pointed by Cattran et. al. on the basis of the Toronto Registry study, with a follow-up of 184 unselected non-azotaemic patients with $\mathrm{IMGN}$, that the duration of heavy proteinuria $[5,6]$ is an independent risk factor for renal insufficiency.

In the present study we addressed two questions: how does membranous glomerulonephritis present and is the chronicity of proteinuria a risk factor for renal insufficiency, as follows. Renal biopsies were taken from all patients presenting with signs of symptoms suggestive of idiopathic glomerular disease on the basis of strict indications by the nephrologist of the participating regional hospitals. Next, patients entered the follow up. Results show that almost all patients with membranous glomerullonephritis present with non-azotaemic normotensive nephrotic syndrome and histological stage 1 lesions; follow-up shows chronic heavy proteinuria to be a risk factor for renal insufficiency in these patients.

\section{Patients and methods}

Patients derived from a prospective epidemiological regional study executed between 1978 and 1988 and designed to chart the incidence and natural history of idiopathic glomerular disease [7]. Briefly, renal biopsies were taken on the basis of the following indications in hierarchical order: recent onset uraemia (serum creatinine concentration $>300 \mu \mathrm{mol} / \mathrm{l})$ and recent onsel nephrotic syndrome $(24$ urinary protein excretion $\geq 5.0 \mathrm{~g} / \mathrm{day}$, in the absence of Bence Jones proteinuria). Recent onset implied that renal biopsies were taken within 6 months after discovery 
of signs and symptoms of renal disease. The other indications were chronic erythrocyturia or recurrent macroscopic hematuria and chronic proteinuria ( 24 hour protein excretion $\geq 0.5 \mathrm{~g} / \mathrm{day}$ ) in the absence of chronic hypertension (blood pressure $\leq 140 / 90 \mathrm{mmHg}$ ) of at least six months duration; here renall biopsies were taken within 2 years of patient identification. The first part of the study was carried out between 1978 and 1984 and the second part, in which more hospitals participated, between 1984 and 1987 . In each period renal biopsies were taken out of a total adherent population of $1.5 \times 10^{6}$ adults between 16 and 65 years at risk, in the second period renal biopsies were taken out of a total adherent population of $1.0 \times 10^{6}$. Patients with systemic disease were excluded [7].

Included in the present study were the patients with membranous glomerulorephritis, biopsied because of any of the indications cited above. The diagnosis idiopathic membranous glomerulonephritis required the absence of systemic disease at the time of renal biopsy and the first year of follow up. The ANF and rheumatoid factor had to be negative, and the liver function tests had to be normal.

At the time of renal biopsy and at follow up at least twice a year serum creatinine concentration, urinary creatinine and protein excretion was measured. Proteinuria was measured by the Biuret method. Complete and partiall remission was defined as proteinuria $<0.5 \mathrm{~g} /$ day and $0.5-2.0 \mathrm{~g} /$ day, respectively, in the presence of a creatinine clearance of $\geq 60 \mathrm{ml} / \mathrm{min} / 1.73 \mathrm{~m} 2$.

All patients were treated with diuretics: no prophylactic oral anticoagulation was given. Anticoagulation was given when thrombo-embolic episodes occurred. When desired patients were treated with indomethacin $150 \mathrm{mgr} /$ day for a 2 year period commencing at the time of renal biopsy; when indomethacin was not tolerated short courses of corticosteroids - 4 weeks not exceeding $1 \mathrm{mg} / \mathrm{kg}$ - could be given. Indomethacin was stopped when the serum creatinine concentration exceeded 120 $\mu$ mol/l or when side effects occurred. One participating physician treated, contrary to protocol, three patients with cyclosporine $A$, these were excluded from follow-up. Patients were followed up for a mean of 11 years (range 8 - 14 years) or until endstage renal disease developed.

All renal biopsies were processed and stained for light microscopy, transmission electron microscopy, and immunohistochemistry as described elsewhere [7]. All renal biopsies were stained with ant-Hbe; Hbe had to be absent from the glomeruli for the membranous glomerulonephritis to be considered idiopathic. Renall biopsies were classified histologically using silver methenamine 
hematoxylin and eosin stains of 1 to 2 micron sections according to Noel [1], where stage 1 is no subepithelial spikes or small spikes seen focally and segmentally only, stage 2 is the presence of diffuse subepithelial spikes, and stage 3 incorporation of the deposits into the altered glomerular basement membrane. Transmission electron microscopy was done on all to confirm or refine the light microscopic classification according to Churg et al [8], specifically the identification of stage III, IV and V disease.

\section{Statistics}

Statistical analysis was performed on a SPSS programme for Windows. Correlations were determined calculating correlation coefficients corrected for known risk factors for renal failure, as age, gender, initial proteinuria, initial creatinine clearance and type of therapy. Linear regression analysis was used to independence of individual prognostic factors on disease progresision.

\section{Results}

\section{Incidence of idiopathic membranous glomerulonephritis}

The incidence of idiopathic membranous glomerulonephritis presenting with apparently acute nephrotic syndrome was remarkably constant over each study period, when expressed as the number of patient per $1.0 \times 10^{6}$ adherent adult population at risk (table 1). Thus 14 patients who presented with recent onset nephrotic syndrome were identified in the period $1978-1984$ per $1.5 \times 10^{6}$ adults at risk and another 10 in the period 1984 - 1987 with $10^{6}$ patients at risk. Excluded from these data are 2 patients with membranous (stage 1) glomerulonephritis who presented with recent onset nephrotic syndrome, clinically of the idiopathic variety, but who were shown during follow up to have bronchial carcinoma $(\times 1)$ and chronic hepatitis $B$ infection $(x 1)$ with normal liver function test at the time of renal biopsy. The latter patient had Hbe-antigen deposited in the glomerular immune complexes.

Of the 18 patients biopsied because of chronic proteinuria of less than 2 years duration with normal renal function (serum creatinine concentration $<120 \mu$ mol/1) and with a normal blood pressure, one had membranous glomenulonephritis (table 2). The 18 patients were middle aged and had either focal and segmental glomerulosclerosis $(n=7$, male : female ratio: $6: 1$ ) or renal wascular hyalinosis 
Table 1. Idiopathic membranous glomerulonephritis, the clinico-pathological presentation. Prospective epidemiological study of the Working Party on Renal Disease in Limburg"

\section{Clinical characteristics}

Number of patients

Male: female

Mean age (range)

Nephrotic syndrome

Chronic proteinuria

Hypertension ( $>90 \mathrm{mmHg}$ )

Serum creatinine $>120 \mu \mathrm{mol} / \mathrm{l}$

Mean proteinuria (gram/day)

\begin{tabular}{cc}
$1978-1984^{1}$ & $1984-1987^{\mathrm{q}}$ \\
\hline 14 & 11 \\
$10: 4$ & $7: 4$ \\
$43(24-64)$ & $44(16-62)$ \\
14 & 10 \\
0 & 1 \\
0 & 1 \\
0 & 0 \\
$7.9 \pm 2.5$ & $6.8 \pm 4.6$ \\
$(5.0-12.2)$ & $(1.8-16.6)$
\end{tabular}

Light microscopy* Noel classification [1]

- Stage 1(a), no spikes

10

6

- Stage 1(b), focal segmental spikes)

4

4

0

1

0

0

0

0

\section{Legend to the table}

${ }^{5}$ Indications for renal biopsy in hierarchical order: recent onset uraemia serum creatinine $>300 \mu \mathrm{mol} / /$ ), recent onset nephrotic syndrome (proteinuria $>5 \mathrm{~g} /$ day). Renal biopsies for these indications were taken within six months after onset of signs or symptoms of disease. Chronic haematuria \pm proteinuria (between 0.5 and $5 \mathrm{~g} /$ day) of at least six months duration, but within two years after identification, in the absence of chronic hypertension.

Adherent adult population at risk between 18 and 65 years was $1.5 \times 10^{6}$ in the first period, while $10^{6}$ in the second period

*All renal biopsies showed diffuse glomerular, granular capillary deposition of IgG and $\mathrm{C} 3$ on immunofluorescence. C1q was absent in these biopsies. Staging was performed on 1-2 $\mu \mathrm{m}$ sections of cold buffered, $4 \%$ formaldehyde fixed and paraplast embedded renal biopsies. Sections were stained with silver methenaminhematoxylin and eosin. 
$(n=7$, male : female ratio: $1: 6)$ or normal renal tissue $(n=3)$ immunohistologically and, in one instance, membranous glomerulonephritis with a proteinuria of $1.5 \mathrm{~g} / \mathrm{day}$.

Of the 68 patients with chronic erythrocyturia and proteinuria in excess of 0.5 g/day of less than 2 years duration, of unknown origin and with a serum creatinine concentration less than $300 \mu \mathrm{mol} / \mathrm{l}$, none had idiopathic membranous glomerulonephritis.

Table 2. Renal immunopathology in nephrotic syndrome and chronic proteinuria $^{1}$

nephrotic syndrome chronic proteinuriag

recent onset late $<2$ yrs $>2$ yrs

\section{Diagnosis}

Membranous GN:

- Idiopathic

2

1

- secondary

2

Minimal change GP 14

FSGS

Renal vascular

hyalinosis

Normal renal

tissue

Divers $^{\circ}$

Legend to the table:

Nephrotic syndrome: excretion of serum proteins $>5.0 \mathrm{~g} /$ day in the absence of Bence Jones proteinuria, recent indicated that biopsies were taken within 6 months of patient identification. Late: nephrotic syndrome of at least six months duration. Chronic proteinuria: excretion of serum proteins $>0.5 \mathrm{~g} / \mathrm{day}$ in the absence of hypertension ( $<140 / 90 \mathrm{~mm}$. Hg) and azotaemia (serum creatinine $<120 \mu \mathrm{mol} / 1$ ); renal biopsies were taken within 2 years of detection of proteinuria $(n=10)$ or after 2 years $(n=5)$

"FSGS: focal and segmental glomerulosclerosis

Divers: includes acute glomerulonephritis, membranoproliferative glomerulonephritis, chronic interstitial nephritis with FSGS, IgA nephropathy, AL amyloidosis, focal and segmental pauci-immune extracapillary glomerulonephritis and diffuse mesangial proliferative glomerulonephritis. 


\section{Indication for renal biopsy and histological staging}

Since in retrospective [1,2] and prospective studies [3] on the natural history of idiopathic membranous glomerulonephritis patients presented not only with recent onset, but also with chronic nephrotic syndrome, mild proteinuria or uraemia, we assessed whether our indications for renal biopsy excluded such patients as follows. In the first 2 years of this study $(1978-1980) 12$ renal biopsies were taken because of chronic nephrotic syndrome $(n=4)$, chronic proteinuria $(n=5)$ or chromic uraemia $(n=3)$ from patients suspected of having idiopathic glomerular disease, but with a duration of signs of symptoms well theyond the inclusion criteria of this study; hence they were excluded from the analysis of the incidence of disease [7]. Of these 12 patients three had membranous glomerulonephritis stage 3 , presenting with nephrotic syndrome over 1 years duration $\left(x_{2}\right.$ ) or chronic uraemia (x 1) (table 3 ).

Renal biopsies, taken because of any indication cited above in the inclusion criteria, yielded 25 patients with idiopathic membranous glomerulonephritis. Of these patients 24 had stage 1 histology in the absence of tubulo-interstitial changes and all presented with recent onset nephrotic syndrome; only one patient was hypertensive and all had a normal serum creatinine concentration. One patient with stage 3 membranous glomerulonephritis without tubulo-interstitial changes presented with chronic mild proteinuria. In all biopsies the glomerular immune aggregates stained immunohistochemically for polyclonal IgG and complement components C3 and C5b-9 neoantigen (table 2).

\section{Natural history of disease}

Twenty-four patients with recent onset nephrotic syndrome and stage 1 histology entered the follow up. Three patients were, contrary to therapeutic protocol, treated by one participating physician with cyclosporine $A$ and therefore excluded from analysis; of these cyclosporine. A treated patients one died shortly after renal biopsy because of a cerebrovascular accident, one went into partial remission at 2 years follow up and the third, with persisting nephrotic syndrome, became dialysis dependent within a 2 year period.

The other patients (see addendum) were treated with diuretics $(n=5)$ only or with indomethacin ( $n=16) 150 \mathrm{mg} /$ day for the first 2 years. Oral anticoagulation was only given when a thrombo-embolic episode occurred $(n=3)$. Indomethacin was stopped before 2 years of therapy, because of gastrointestinal complaints $(n=2)$, because of partial or complete remission $(n=5)$ or because of an increase in serum creatinine concentration over $120 \mu \mathrm{mol} / \mathrm{(n}=4)$. Three patients were treated with 
steroids, after cessation of indomethacin, without influencing the nephrotic syndrome. At 5 years follow-up the incidence of partial or complete remission in the patients treated with diuretics only ( 4 of 5 ) did not differ significantly from those treated with indomethacin $(11$ of 16$)(p=>0.13)$.

Table 3. Membranous glomerulonephritis: indication for renal biopsy and histological staging ${ }^{1}$

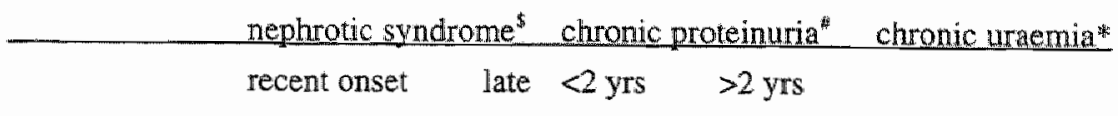

stage 1

stage 2

stage 3

21

1

Legend to the table:

Noel classification: stage 1: no spikes or focal and segmental spikes only, stage 2: diffuse subepithelial spikes, stage 3 : glomerular basement membrane remodelling \pm inmune deposit resolution

${ }^{8}$ Recent onset: renal biopsy taken within 6 months of signs or symptom of disease; proteinuria $>5 \mathrm{~g} /$ day, Late: nephrotic syndrome of at least six months duration

${ }^{*}$ Chronic proteinuria: daily excretion of serum proteins $>0.5 \mathrm{~g} / \mathrm{day}$, in the absence of hypertension $(<140 / 90 \mathrm{mmHg}$ )

$*$ Serum creatinine concentration $>300 \mu \mathrm{mol} / \mathrm{f}$ for at least 6 months

Follow-up (table 4) of patients treated with diuretics, with or without indomethacin, showed the rates of complete remissions at 2,5 and 11 years to be $30 \%, 57 \%$ and $50 \%$ respectively. The rates of partial remissions at 2,5 , and 11 years were $28 \%, 23 \%$ and $19 \%$ respectively. Thus, after 5 years follow up $80 \%$ of all patients were in partial or complete remission. One patient with a partial remission at 2 years developed recurrent nephrotic syndrome at 5 years and went into late renal insufficiency at 12 years of follow-up; repeat renal biopsy in this patient (vide infra) showed Churg IV lesions, focal and segmental glomerulosclerosis, and global glomerular obliteration in the absence of interstitial infiltration histologically and no 
glomerular C3 or C5b-9 deposition immunohistochemically. Of the patients with complete or partial remissions at 5 years follow up three, of whom two with complete remission, developed late recurrences of the nephrotic syndrome; this was treated in one patient, with recurrent nephrotic syndrome after complete remission, with a bolus of intravenous cyclophosphamide $(1000 \mathrm{mg} / 1.0 \mathrm{~m} 2)$ which resulted in complete remission.

Of all patients 4 (19\%) developed chronic renal insufficiency. Analysis of follow-up shows that of the patients $(n=8)$ with persisting nephrotic syndrome at 2 years follow up. two had already developed mild azotaemia (serum creatinine concentration $175-300 \mu \mathrm{mol} / \mathrm{l}$ ) within two years and another developed azotaemia within 5 years, whereas none of the 13 patients with partial or complete remission at two years developed renal falure within 5 years of follow up (table 3 ).

of these 21 patients three experienced thrombotic episodes (poplitial vein $\times 2$, femoral vein $x 1$; ; in two of these this occurred within six months after detecting the nephrotic syndrome and in another late in the history of disease with recurrence of nephrotic syndrome after a complete remission.

In our 21 patients the initial degree of proteinuria at the time of renal biopsy did not predict prognosis. First, the mean initial proteinuria of those patients who went into complete or partial remission at 2 years follow up ( $n=13$, mean proteinuria $6.7 \mathrm{~g} /$ day, range $5-11.2$ grams) did not differ $(\mathrm{p}=0.71)$ from those with persistent nephrotic syndrome $(\mathrm{n}=8$, mean proteinuria $6.8 \mathrm{~g} /$ day, range $5.8-11.2$ grams). Second, of the patients with persisting nephrotic syndrome at two years the initial proteinuria of those going into early or late chronic renal insufficiency $((n=3$, mean proteinuria $6.7 \mathrm{~g} /$ day, range $6.2-7.3$ grams) did not differ from the others ( $\mathbb{n}=5$, mean proteinuria $7.9 \mathrm{~g} /$ day, range $5.8-12.2$ grams) of which 4 of 5 eventually went into complete remission.

The presence of stable nephrotic syndrome at 2 years follow up was associated with a poor renal prognosis, since 3 out of 8 nephrotics developed end-stage renal disease within a 5 year period, whereas none of the 13 patients with partial or complete remission developed renal insufficiency over this period $(p=0.06$, Chi square test). The findings at two years follow-up correlated well with the findings at five year $(R=0.66, p=0.001)$, while age, sex, initial proteinuria, initial therapy and initial creatinine clearance showed no significanrt correlation with follow-up findings at five year. This was confirmed in linear regression analysis. When two patients with persistent nephrotic syndrome and decreased renal function at two year follow-up are not considered, presence of nephrotic syndrome at two year follow-up 
did not differ, in terms of prognosis, from those patients with partial remission at two years, as of the six nephrotics at two years four eventually went into complete remission. One patient with persistent nephrotic syndrome and one patient with partial remission at two years developed late chronic renal insufficiency.

Table 4. Natural history of 21 patients with iMGN, presenting with recent onset nephrotic syndrome. Prospective epidemiological study of the Working Party on Renal Disease in Limburg

Follow-up (in years)

Remission of proteinuria

-complete $(<0.5 \mathrm{~g} /$ day $)$

-partial (0.5-2.0 g/day)

Nephrotic syndrome

End-stage renal disease ${ }^{\text {dx }}$
2

7

6

$8(2)$

0
5

11

4

2

4

\section{Legend to the table}

Renal biopsies were taken within six months of signs or symptoms of disease. All patients were treated with diuretics $(n=5)$ or diuretics with indomethacin $(n=16)$. Renal biopsies all showed histological stage 1 membranous glomerulonephritis: no spikes in 16 patients and focal segmental spikes in 5 patients.

* Defined as proteinuria over 5 g/day per 15 mmol/l urinary creatinine

() Number of patients with creatinine $175-300 \mu \mathrm{mol} /$,

End-stage renal disease: serum creatinine $>300 \mu \mathrm{mol}$

\section{Repeat renal biopsies}

Four repeat renal biopsies were taken. One was taken because of persisting nephrotic syndrome and decreasing renal function (no 38/85) after 1 year follow up and showed stage 2-3 membranous glomerulonephritis and a diffuse interstitial mononuclear infiltrate (fig.1) histologically; the glomerular immune deposits stained for IgG, C3 and MAC. Another biopsy was taken because of stable proteinuria ( 3.5 g/day) and decreasing renal function after 9 years follow up; this patient had recurrent nephrotic syndrome after 5 years follow up after an initial partial remission 
(no 40/79). This biopsy showed Churg IV glomerular disease, global glomerulosclerosis with interstitial fibrosis and tubular atrophy and focal and segmental glomerulosclerosis (fig 2); the glomeruli failed to stain for C3 or MAC immunohistochemically and recently formed immune aggregates were not seen by transmission electron microscopy.

Two renal biopsies were taken after 9 and 11 years of follow up because of persisting mild proteinuria of $1.2 \mathrm{~g} /$ day (no $2 / 83$ ) and persisting severe proteinuria (4.5 g/day) after previous partial remission (no 44/81). Both had normal renal function and in both instances no tubulo-interstitial changes were seen in the renal biopsy; the glomenuli showed GBM remodelling and were ultrastructurally classified as Churg $V$ and IV, respectively (fig 3). No C3 was demonstrable immunohistochemically in these glomeruli.

\section{Discussion}

The main findings of this prospective regional study are four fold. First, the incidence of idiopathic membranous glomerulomephritis is remarkably constant over a prolonged period of obserwation, when renal biopsies are taken on the basis of strict indications from all patients presenting with signs or symptoms suggestive of idjopathic glomerullar disease. Second, almost all newly identified patients present with a proteinuria $\geq 5.0 \mathrm{~g} / \mathrm{day}$, a nomal serum creatinine concentration and normotension. The third observation of interest is that of the patients with persisting nephrotic syndrome of two years duration $37 \%$ will develop end-stage renal disease. Finally, repeat renal biopsies are required to decide whether persisting nephrotic syndrome or proteinuria is immune mediated.

It is clear that this study underestimates the incidence of membranous glomerulonephritis by excluding patients over 65 years of age. Such patients are included in other studies on the natural history of disease 12,3$]$; e.g. of the 100 patients in one study, 50 were over 50 years of age and 13 over 70 years of age [3]. "The mean age of our patients on the other hand is similar to that of the French [1] and the Toronto Registry study [5]; our study yields incidence rates of male : femalle of $2: 1$, which is identical to the sex ratios observed by others $[2,3,5]$, but differs from the French study [1] which includes relatively many females of a young age (ratio male: female $=1: 1$ ). 
Our study differs from other retrospective $[1,2]$ and prospective [3] studies on the natural history of disease, because almost all our patients had stage 1 histology, a normal blood pressure and serum creatinine concentration, and proteinuria $\geq 5.0$ $\mathrm{g} /$ day $/ 1.73 \mathrm{~m} 2$ at presentation. Although the number of patients with stage 1 histology in our study is small, it is equal to that of the French [1] retrospective study ( 22 of 114 patients), the Italian prospective study (24 of 100 [3]) and exceeds those of the retrospective study of the Mayo clinic (7 of 89 [2]); it differs conspicuously from these studies, however, because of the absence of stage 2 lesions and the virtual absence of stage 3 lesions. Stage 3 lesions were also seen by us in patients with relatively long standing disease, who were excluded by the design of the study which aimed to determine the incidence of disease [7]. The study, in addition, minimised doctor tot doctor delay by avoiding a centre effect: the referral of patients for renal biopsy to a centre or referral of the prognostically poor patients to a centre. Also, the Dutch Health Care system insures easy access of the public to the general practitioner, quite apart from routine urine analysis because of draft, sport or insurance check-ups and hence reduces patient to doctor delay. It follows that in other studies patients further advanced in the natural history of disease were included, which is illustrated by the greater number of stage 2 and 3 lesions [1-3]. It implies that risk factors for renal insufficiency like age, hypertension and impaired renal function at inclusion of the patients [2] are not independent risk factors, but rather reflect chronicity of disease or referral of a prognostically less benign subset. Conversely, the good renal prognosis observed in an Italian prospective study [3] may be influenced by the fact that $24 \%$ of the included patients had normal renal function with a proteinuria $0.2-2.0 \mathrm{~g} / \mathrm{day}$, which is compatible with the view that they had entered partial or complete remission at the time of inclusion.

Follow up of our patients showed early partial or complete remission in the majority of patients - with the highest rate of remission $(80 \%)$ at 5 years follow-up-, stable nephrotic syndrome over two years duration in $38 \%$, late recurrences of nephrotic syndrome in $14 \%$ and early and late chronic renal failure in $19 \%$. The severity of proteinuria at the time of the first renal biopsy had no bearing on prognosis, for it did not differentiate between those patients going into early remission and those developing early renal insufficiency. This agrees with the observation of Pei et. al. [5] that the chronicity of proteinuria (e.g. $\geq 6.0 \mathrm{~g} /$ day for a 9 month period) and not the degree of it is the best prediction for chronic renal insufficiency. This observation was based on 184 patients from the Toronto Registry followed up for at least 1 year (mean follow up 5.0 years), after excluding those with 
a creatinine clearance $<60 \mathrm{~min} / \mathrm{ml} / 1.73 \mathrm{~m} 2$ regardless of medication, inclusive immunosuppression. This observation also held when applied to the Helsinki $(n=$ 78) and the Milan $(n=101)$ patients [6]; the positive predictive value for chronic renal insufficiency was naturally enhanced when a decrease in renal function was added to the chronic proteinuria as risk factor for chronic renal insufficiency. When the chronicity of nephrotic syndrome in our patients is considered it appears associated with a poor renal prognosis. However, in the absence of early renal failure a persistent nephrotic syndrome over two years duration carried a good renal prognosis. A small subset of patients (in our series 10\%) will develop - independent of the severity of initial proteinuria - early renal insufficiency associated with an interstitial cellular infiltrate at biopsy (unpublished observation and vide infra), whereas another subset with persistent nephrotic syndrome or partial remission will develop late renal failure, but not necessarily through a similar mechanism.

It is clear that - given the observation that persisting severe proteinuria, certainly when associated with decreasing renal function, is a risk factor for or heralds progression into chronic renal insufficiency - it becomes essential to determine whether the proteinuria is immune mediated before initiating immunosuppressive therapy, be it with cytostatic drugs, like oral cyclophosphamide or chlorambucil (see review by [9]), and azathioprine [10], or intravenous gammaglobulin [11], or cyclosporine [12]. In order to avoid taking repeat renal biopsies, attempts have been made to differentiate immune mediated proteinuria from "fibrotic" renal disease by determining urinary $C 5 \mathrm{~b}-\mathrm{C} 9 \mathrm{~b}$ neoantigen or MAC complex [13] and transforming growth factor $\beta 1$ respectively [14]. The determination of urinary MAC complex derives from studies on passive Heymann nephritis, where it was shown ex vivo that the MAC complex is furst inserted in the epithelial cell membrane, next selectively endocytosed and transported across the epithelial cell, in order to be exocytosed in Bowman"s space [15]; it was also shown in this rat model that the urine contained MAC complex [16]. Clinically, urinary MAC does not indicate an exclusive glomerular origin of MAC, since glomerularly filtered C5 can be converted via the alternative pathway by the tubular cells locally into $\mathrm{MAC}[13]$; but even when this is corrected for by measuring urinary $\mathrm{C} 5$ together with MAC [17], less than $20 \%$ of the patients with membranous glomerulonephritis had increased MAC excretion. Measuring urinary TGF- $\beta$ concentrations, on the other hand, indicates the presence of an interstitial cellular infiltration, but does not reflect duration of disease, renal function or the extent of glomerular basement membrane remodeling [14]. Hence, renal biopsies are required to decide whether 
persistent nephrotic syndrome is immune mediated (presence of $\mathrm{C} 3$ and MAC in the immune deposits with/without an active interstitial mononuclear inflitration); this is shown by our study where one early repeat renal biopsy taken because of persistent nephrotic syndrome showed $\operatorname{IgG}$ and complement in the glomerular deposits, whereas the other late repeat biopsies showed glomerular basement membrane remodelling with resolved deposits, without or with focal and segmental glomerulosclerosis and the absence of glomerular C3 or MAC deposition or interstitial cellular infiltration.

In the present study two patients had venous thrombosis (poplitial vein $\times 2$ ) at the time of the first renal biopsy, and one had a thrombosis of the femoral vein at the time of recurrence of nephrotic syndrome after previous complete remission. Thus, thrombo-embolic episodes occurred in $14 \%$ of our patients early after onset nephrotic syndrome. This supports the notion that prophylactic anticoagulation, rather than initiating anticoagulation after a thrombo-embolic event, is the therapy of choice in these patients, outweighing the potential risks of bleeding [18].

\section{Figure 1}

Repeat renal biopsy after 1 year follow up because of declining renal function and persistent nephrotic syndrome.
A. Histology. Silver methenamine HE (x 145). A diffuse mononuclear cellular infiltration is seen: the glomeruli show stage $1 \mathrm{~b}$ membranous glomerulonephritis (Noel staging).

B. Transmission electronmicroscopy; uranyl acetate and lead citrate staining ( $x$ 12.190). Capillary loop with stage $I$, II and occasional early stage III lesion (Churg staging).

C. Immunohistochemistry staining with FITC labeled anti-C3b in the direct technique $(x$ 165). Strong staining of $C 3$ in a capillary pattern. 
Chapter 5

Figure 1
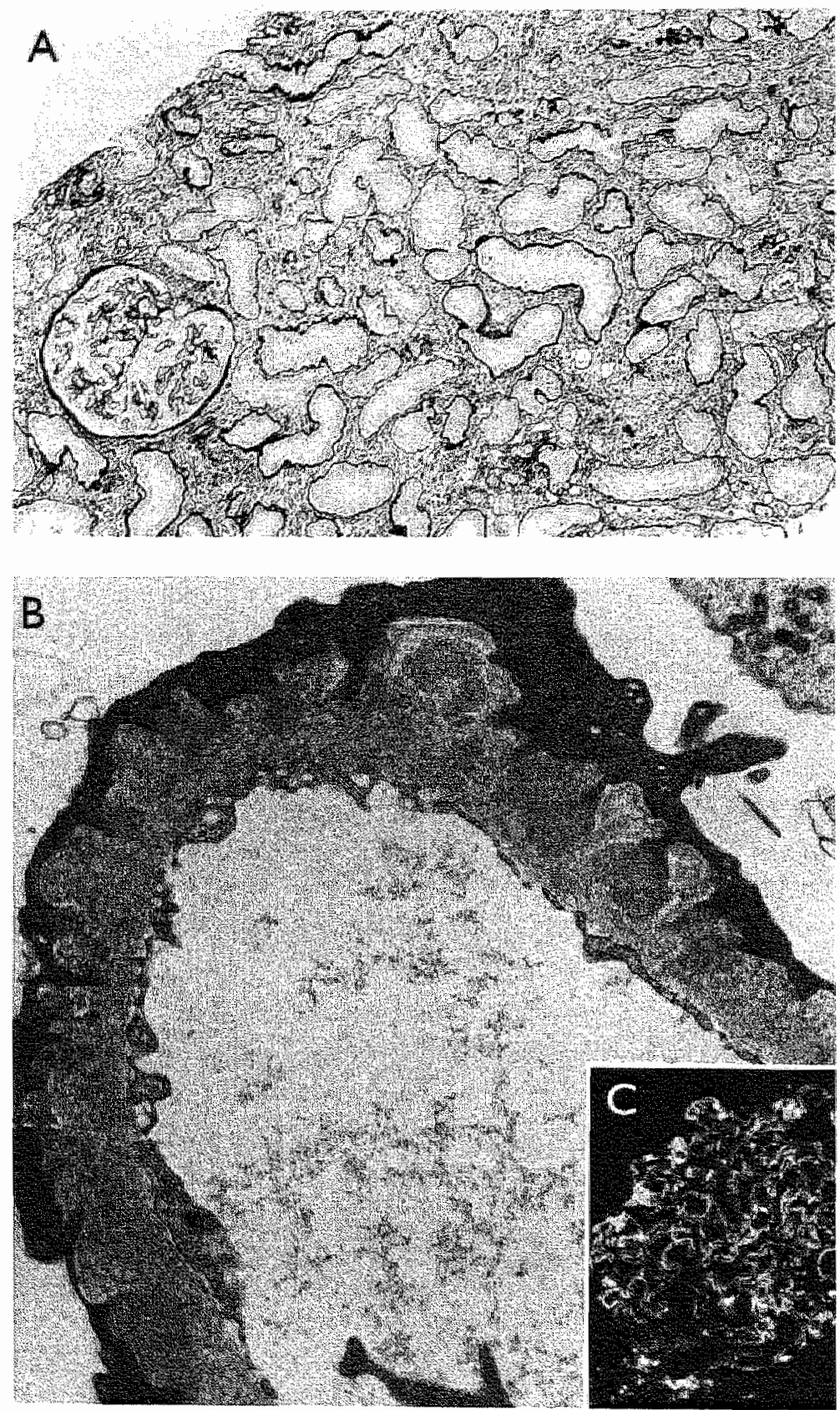


\section{Figure 2}

Recurrent nephrotic syndrome at 5 years follow up after previous partial remission, repeat renal biopsy taken because of persistent nephrotic syndrome with declining renal function at 9 years follow up.

A. Histology. Silver methenamine HE ( $x$ 145). Focal and segmental glomerulosclerosis (left handcorner); global glomerular obliteration and interstitial fibrosis are also present. No active mononuclear infiltrate is seen.

Immunohistochemistry - not shown - revealed the presence of IgG in a capillary, granular pattern but no $\mathrm{C} 3$ deposition.

B. Transmission electronmicroscopy; uranyl acetate and lead citrate staining ( $\mathrm{x}$ 15.290). Non-ischaemic glomerulus. Resolubilised and some non-resolubilised electron dense aggregates are seen within an altered glomerular basement membrane (Churg TV).

C. Histology. Silver methenamine HE (x 165). A non-ischaemic glomerulus is seen: showing stage IV (Churg) lesions. 
Chapters

Figure 2
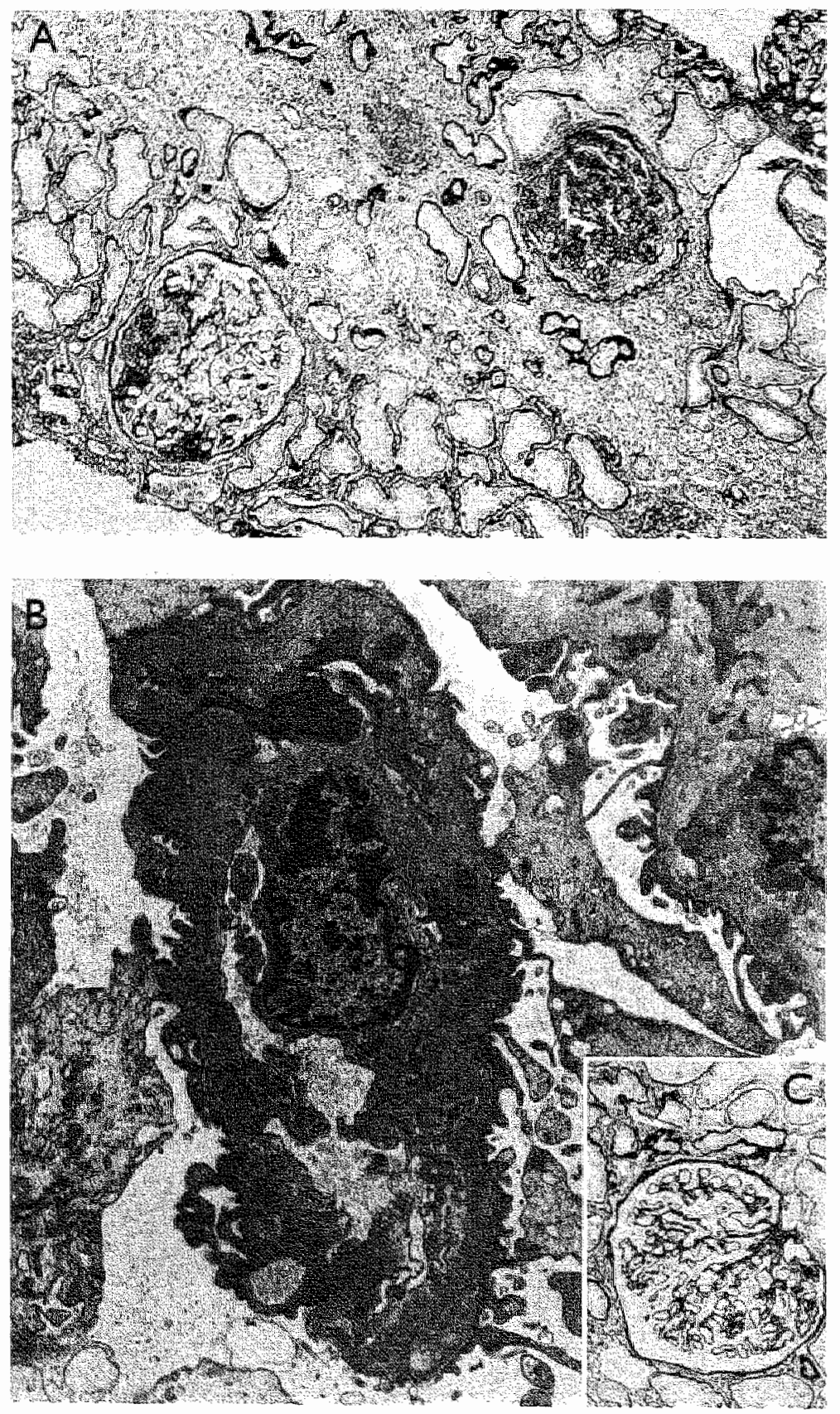
Figure 3

Repeat renal biopsy taken at 9 years follow up because of persisting proteinuria after partial remission at 2 years follow up. Normal renal function at the time of renal biopsy.

A. Histology. Siver methenamine $\mathrm{HE}(x) 360$ ). Histologically the renal biopsy showed a normal architecture. At higher magnification, however, the glomerular basement membrane shows areas of sil ver negative small "punched out" holes. B. Immunofluorescence; FITC labeled (xenogenic) anti IgG in the direct technique. The glomerular basement membrane is stained in a linear, sometimes faintly gramular pattem. Staining for C3 or MAC complex was negative.

C. Transmission electronmicroscopy; uranyl acetate and lead citrate (x 7130). An irregular, remodelled glomerular basement membrane is seen with electron lucent areas, showing the previous presence of resolubilised electron dense aggregates. 
Chupter 5

Figure 3
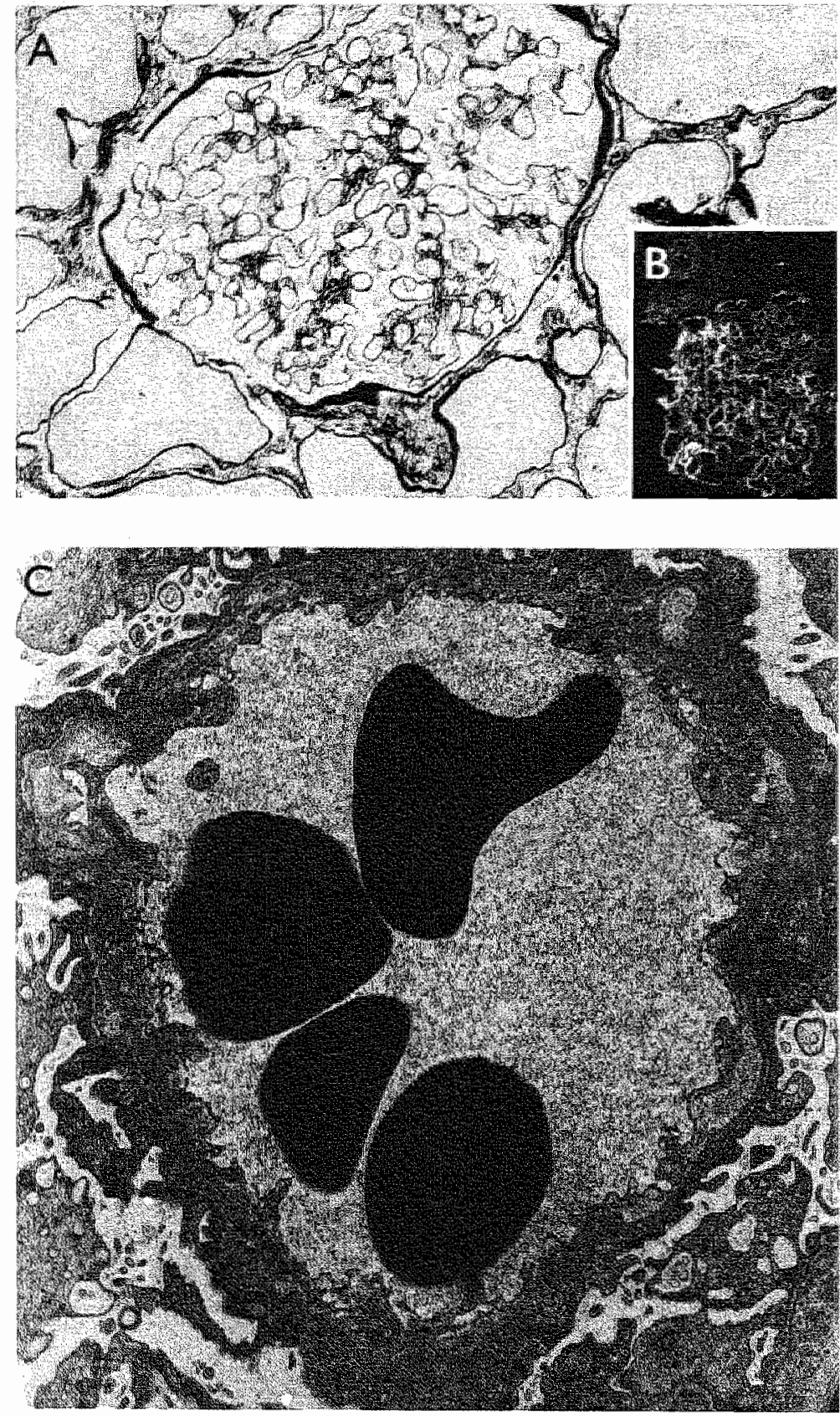


\section{References}

1. Noel L., Zanetti M., Droz D., Barbanel C Long-term prognosis of idiopathic membranous glomerulonephritis. Am J Med 1979; 6: 82-90.

2. Donadio J., Torres V., Velosa J., Wagoner R., Holley K., Okamura M., Ilstrup D., Che C. Idiopathic membranous nephropathy: the natural history of untreated patients. Kidney Int 1988; 33: 708-715.

3. Schiepatti A., Mosconi L., Perna A., Mecca G., Bertani T., Garattini S., Remuzzi G. Prognosis of untreated patients with idiopathic membranous nephropathy. N Engl J Med 1993; 329: 85-89.

4. Reichert L., Koene R., Wetzels J. Prognostic factors in idiopathic membranous nephropathy. Am J Kid Dis 1998; 31: 1-11.

5. Pei Y., Cattran D., Greenwood CMT. Predicting chronic renal insufficiency in idiopathic membranous glomerulonephritis. Kidney Int 1992; 42:960-966.

6. Cattran D., Pei Y., Greenwood C., Ponticelli C. Passerini P., Honkanen E. Validation of a predictive model of idiopathic membranous nephropathy: its clinical and research implications. Kidney Int 1997; 51: 901-907.

7. Tiebosch A., Wolters J., Frederik P., van de Wiel T., Zeppenfeldt E., van Breda Vriesman P. Epidemiology of idiopathic glomerular disease: A prospective study. Kidney Int 1987; 32: 112-116.

8. Churg J., Bernstein J., Glassock R.: Renal Disease (second edition). Tokyo-New York, Igaku-Shoin, 1995.

9. Reichert L., Huysmans F. , Assmann K., Koene R. Preserving renal function in patients with membranous glomerulopathy. Ann Int Med 1994; 121: 328-333.

10. Ehrenreich T., Porush J., Churg J., Garfinkel L., Glabman S., Goldstein M., Grishman E., Yunis S. Treatment of idiopatthic membranous nephropathy. $N$ Engl J Med 1976; 295: 741-746.

11. Palla R., Ciram C., Panichi V., Bianchi A., Parrini M., Grazi G. Intravenous immunoglobulin therapy of membranous neptiropathy: efficacy and safety. Clin Nephrol 1991; 35: 98-104.

12. Cattran D., Greenwood C. Ritchie S., Bernstein K., Churchill D., Clark W., Morrin P., Lavoie S., the Canadian Glomerulonephritis Study Group. A controlled trial of cyclosporine in patients with progressive membranous nephropathy. Kidney Int 1995; 47: 1130- 1135 . 
13. Ogrodowski J., Hebert L., Sedmak D., Cosio F., Tamerius J., Kolb W. Measurement of SC5b-9 in urine in patients with the nephrotic syndrome. Kidney Int 1991; 40: 1141-1147.

14. Honkanen E., Teppo A., Törnroth T., Groop P., Grönhagen-Riska C. Urinary transforming growth factor-1 in membranous glomerulonephritis. Nephrol Dial Transplant $1997 ; 12: 2562-2568$.

15. Kerjaschki D., Schulze M., Binder S. Kain R., Ojha P., Susani M., Horvat R., Baker $P$., Couser W. Transcellular transport and membrane insertion of the C5-9 membrane attack complex of complement by glomerular epithelial cells in experimental membranous nephropathy. J Immunol 1989; 143: 546-552.

16. Pruckno C., Bexens M., Schulze M., Johasm R. Baker P., Couser W. Urinary excretion of $\mathrm{C} 5 \mathrm{~b}$ - C9 reflect disease activity in passive Heymann nephritis. Kidney Int 1989; 36: 65 - 71.

17. Schulze M., Donadio J., Pruchno C., Baker P., Johnson R., Stahl R., Watkins S., Martin D., Wurzner R., Gotze O., Couser W. Elevated urinary excretion of the C56-9 complex in membranous nephropathy. Kidney Int 1991; 40: 533-538.

18. Sarasin F., Schifferli J. Prophylactic oral anti-coagulation in nephrotic patients with idiopathic membranous nephropathy. Kidney Int 1994; 45: 578 - 585. 
Narural History of Idiopathic Membranous Glome rulonephritis: 

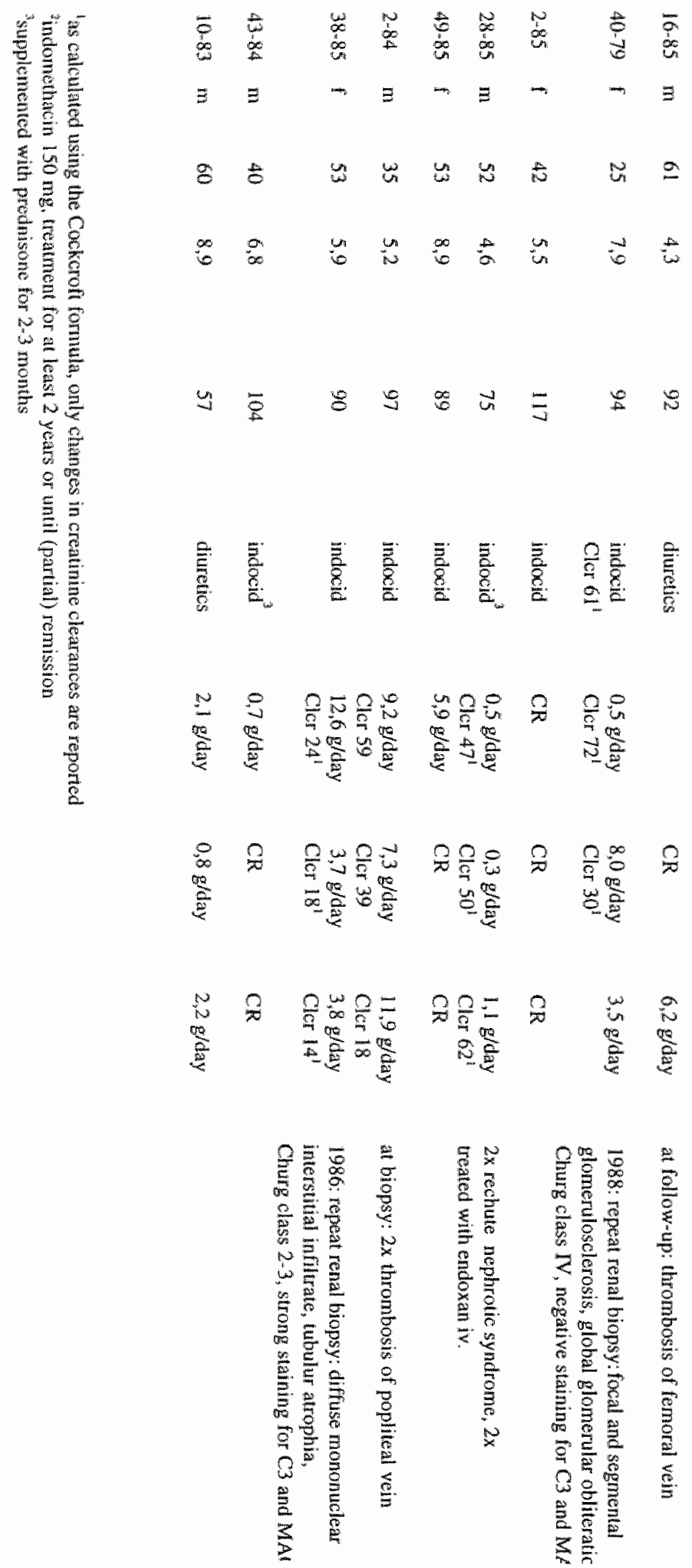


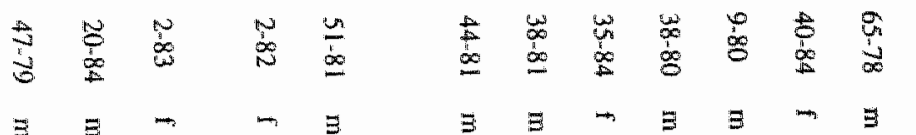

$$
\begin{aligned}
& \text { w }
\end{aligned}
$$

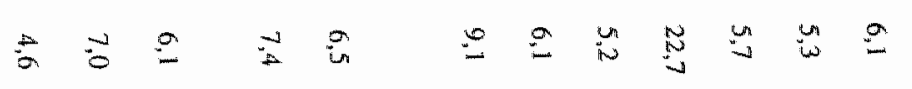

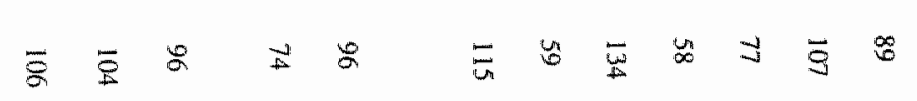

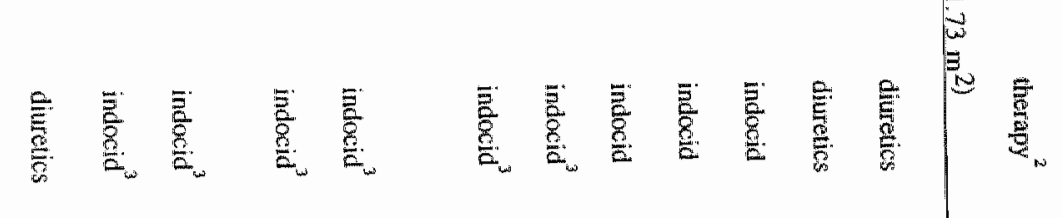

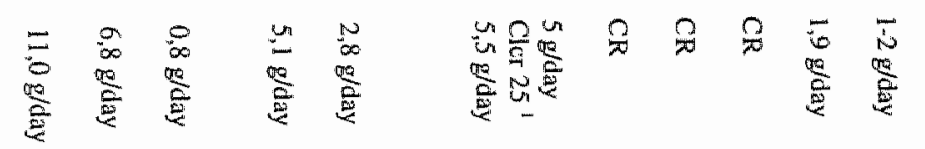

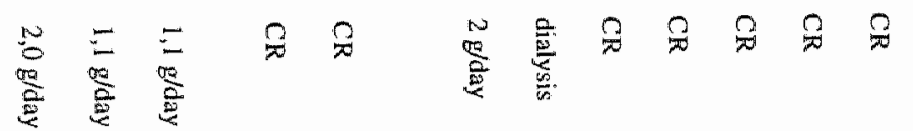

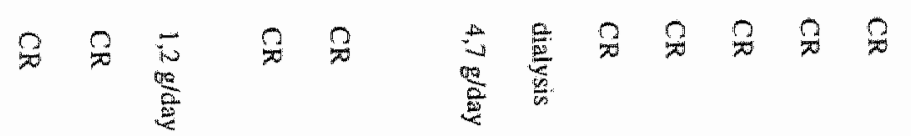

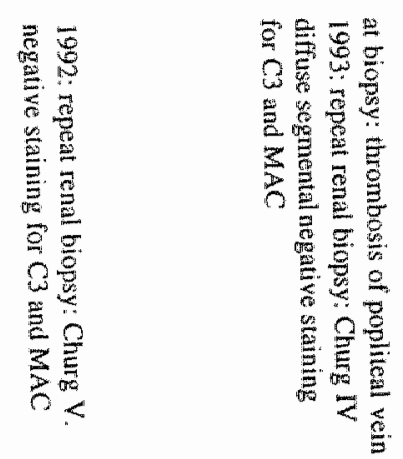

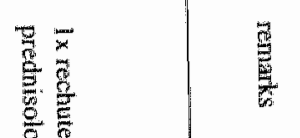

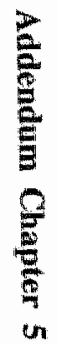

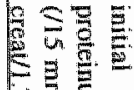

$$
\begin{aligned}
& \text { 㱐 } \\
& \text { N }
\end{aligned}
$$

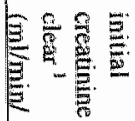

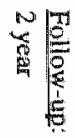

$$
\begin{aligned}
& \breve{\$}
\end{aligned}
$$

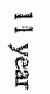

$$
\begin{aligned}
& \text { t. }
\end{aligned}
$$




\section{Summary}


This thesis was designed to outline the natural history of idiopathic glomenulonephritis and to identify risk factors for a poor renal prognosis early in the natural bistory of disease. Patients were all included shortly after patient identification, thus in an early stage of disease, in a prospective regional study. The study outline ruled out a centre-effect, avoiding concentration of patients with an unfavourable prognosis in academic centres. Reviewing current information on patients who enter renal replacement programmes, it is clear that idiopathic glomenulonephritis will remain the cause of renal insufficiency in a substantial number (roughly $20 \%$ ) of patients. For treatment of idiopathic glomerulonephritis and tertiary prevention purposes, clinical decision-making should be based on knowledge of the natural course of disease. Key symptoms that herald a poor prognosis should be timelly recognised and lead to a proper choice in therapy. The treatment options in idiopathic glomerulonephritis consist of immunosuppressive therapy (prednison with or without cyclophosphamide, cyclosporine, chlorambucil), immunomodulating therapy (such as intravenous immunoglobulin treatment) or symptomatic treatment. The symptomatic therapy includes treatment of hypertension, preferably with $\mathrm{ACE}$-inhibition, restriction of protein when slight renal failure is present, and treatment of hyperlipidemia, hyperparathyroidism. The key question in management of idiopathic glomerulonephritis remains the exact timing of which therapy when to start. Repeat renal. biopsies can provide valuable information on the cause of persisting symptoms of renal disease or progression and thus influence this decision.

In Chapter 1 we discuss the literature of idiopathic haematuria and nephrotic syndrome, especially concerning the role of renal biopsy in these syndromes. In idiopathic non-proteinuric haematuria, where differentiation of urological from glomerular causes is the main problem, current clinical methods, like quantitation of dysmorphic erythrocytes in the urine or determination of serological parameters (e.g. circulating immune complexes) are not sufficient. Renal biopsies are needed to establish the diagnosis, and - as in the case of $\operatorname{IgA}$ nephropathy - might reveal information on the prognosis of the individual patient. Furthermore, in establishing a correct diagnosis unnecessary invasive urological examinations are avoided. In membranous glomerulonephritis, a common cause of nephrotic syndrome in adults, renal biopsies do not give information about prognosis, but immune activity may be determined in immunofluorescence studies, guiding decisions about treatment. In contrast to nephrotic syndrome in children, blind immunosuppressive treament is less successful, as mimimal change nephropathy is less prevalent in adults, and is less 
prednisone-sensitive. Reviewing the data in chronic renal allograft rejection, we conclude that early in the course of renal disease immune-mediated mechanisms are probably responsible for histological damage and clinical symptoms, while at a certain point in time non-immune-mediated processes are responsible for ongoing injury. Studies in chronic renal allograft rejection have clearly indicated that removal of an immunological drive (alloantigen driven) by re-transplantation into the original donor does no longer abolish renal disease progression when a critical period has elapsed. Clearly, immune and non-immune mechanisms are responsible for disease progression.

In Chapter 2 we discuss the results of a prospective study on the natural history of 49 adult patients (12 primary IgA nephropathy, 13 thin GBM nephropathy, 20 normal renal tissue and 4 miscellaneous nephropathies) who presented with idiopathic non-proteinuric non-azotaemic haematuria of at least six months duration, in the absence of hypertension and with a negative urological work-up. It has previously been shown that clinical findings at presentation are not helpful in the differential diagnosis of patients with chronic haematuria, as patients with urological causes of bleeding could not be distinguished from those with glomerular haematuria. Median follow-up in this study was 11 years with a range of 8-14 years. At the end of followup renal function had remained stable in all subsets except for those with miscellaneous disease. Haematuria was still present in all patients with thin GBM nephropathy, in all but two patients with IgA nephropathy who went into immunopathological remission, in three out of four miscellaneous nephropathies and in seven out of 20 patients with normal renal tissue. Of the latter patients five had a history suggestive of urolithiasis at follow-up, which was in the absence of hypercalciuria and hyperuricosuria. The cause of haematuria corresponded well with the persistence of haematuria: glomerular causes led to persistence of symptoms, while non-nephrological causes showed disappearance of haematuria during follow-up. Seven thin GBM patients, five IgA nephropathy patients and three miscellaneous nephropathies developed hypertension; the incidence of hypertension in each subset was significantly higher than in patients with normal renal tissue. The study shows that in young adults with idiopathic chronic nonproteinuric haematuria of 4 years duration, renal biopsy will give a definite diagnosis in $86 \%$ of the patients, and that those patients with so-called minor glomerular diseases are at high risk for hypertension. Those patients with normal renal tissue have a high incidence of urolithiasis and should have an urological follow-up. 
In order to differentiate early Alport syndrome from thin GBM nephropathy we describe in Chapter 3 the results of a prospective epidemiological study in which 19 normotensive and non-azotaemic adult patients with chronic microscopic (18 of 19) and macroscopic (1 of 19) haematuria and biopsy-proven thin GBM nephropathy were followed for 12 years median (range 9 to 15 years. All renal biopsies showed presence of the Goodpasture antigen when tested immunohistochemically. Presence of Alport syndrome was excluded clinically as none of the patients had complaints of hearing loss or abnormalities by audiography and ophthalmology. Thus, thin GBM lacked the extrawrenal symptoms of Alport syndrome and did not fulfil the clinical and histological criteria. At the end of follow-up, the incidence of hypertension in thin GBM nephropathy (35\%) exceeded that of healthy clinical controls $(p=0.048)$, one of the hypertensives developed mild renal failure. Of our thin GBM patients five presented with significant proteinuria (> $250 \mathrm{mg} /$ day), while this increased during follow-up in another two; this increased proteinuria may associated with the presence of focal and segmental glomenlosclerosis in renal biopsy. In the normotensive patients, the glomerular filtration rate at follow-up as measured by inulin clearance, was reduced in 3 out of 7 ; these were over 50 years of age. ). Renal biopsies of thin GBM patients at entry showed an increased incidence of focal global glomerulosclerosis when compared to disease controls as IgA nephropathy ( $\mathrm{p}=0.047$ ) and normal renal tissue $(\mathrm{p}=0.075)$. We postulate that the increased incidence of hypertension, development of proteinuria and decreased GFR and ERPF is related to the increased degree of focal global glomerulosclerosis as seen in the initial biopsy and repeat renal biopsies of the thin GBM patients. The fact that decreases in GFR and ERPF were seen in patients who had remained normotensive, suggest that a renal substrate is responsible for the loss in renal function. Histologically, focal global glomerulosclerosis with tubular atrophy and interstitial fibrosis appears a likely substrate. Although no family members were known to have renal disease at inclusion, within four families six elderly first degree relatives had developed unexplained renal insufficiency at the end of follow-up. Thus, thin GBM nephropathy predisposes to premature glomerular obsolescence leading in time to increased incidences of hypertension and late onset renal insufficiency.

In Chapter 4 we evaluated the long-term follow-up of 27 normotensive nonazotaemic adult idiopathic IgA nephropathy patients with chronic haematuria of at least six months duration. within 2 years after patient identification. As controls 17 thin GBM patients, 24 patients. with normal renal tissue and 9 patients with miscellaneous 
nephropathies were followed. Median follow-up was 11 years, range 8 to 14 years. Previous studies have identified heavy microscopic haematuria, the absence of gross haematuria, the severity of proteinuria, decreased renal function at the time of renal biopsy and uncontrolled hypertension as risk factors for an unfavourable renal. prognosis. A major drawback of these studies is that patients identified early in the natural history of disease are inevitably mixed with those well on the way to end-stage renal disease. Hence many of the risk factors, e.g. old age, hypertension, azotaemia and severe proteinuria are related, since they are secondary to a slowly decreasing renal mass, histologically characterised by global glomerular fibrosis, focal and segmental glomerulosclerosis, interstitial fibrosis and inflammation and vascular hyalinosis. In our study renal biopsies were scored semi-quantitatively in terms of activity and chronicity indices, using a modified NIH scoring system originally developed for lupus nephritis. During follow-up 2 patients with $\operatorname{IgA}$ nephropathy went into histological remission, 9 IgA nephropathy patients developed signs of progressive renal disease, consisting of hypertension, increased proteinuria, or a combination of both. Three patients developed renal failure, necessitating dialysis in one. Initial proteinuria over 1 gram/day was associated with a high activity score, extracapillary lesions and with late onset of uraemia. Mesangial $\operatorname{IgG}$ deposition and a higher initial chronicity index were associated with development of hypertension during follow-up. Circulating immune complexes, IgG/IgA rheumatoid factor, age and gender had no bearing on disease history. In the multivariate analysis, a high initial chronicity index, erythrocyturia and mesangial IgG deposition are independent determinants of natural history of disease. We conclude that in patients with IgA nephropathy, identified early in the course of disease, erythrocyturia, a high chronicity index and mesangial IgG deposition in the presence of nomal renal function are risk factors for poor renal survival. Disappearance of haematuria is associated with remission of IgA nephropathy immunopathologically and a low activity and chronicity index at initial biopsy.

In Chapter 5 the natural history of idiopathic membranous glomerulonephritis is given; the data derive from the prospective epidemiological study carried out in Limburg between 1978 and 1988. In this study renal biopsies were taken from all patients which presented with signs or symptoms of idiopathic glomerular disease on the basis of strict indications and contra-indications. Patients were followed for more than 10 years. This unique data base shows that idiopathic membranous glomerulonephritis presents almost invariably with recent onset nephirotic syndrome, 
nomotension, nomal renal function and stage 1 histology. Stage 2 and 3 disease were only observed in chronic disease and therefore excluded by the design of the study. This implies that in other studies on the natural history of this disease patients with chronic disease, remissions of the disease, and recent onset disease were all included in the analysis of risk factors for renal insufficiency. Our data show $80 \%$ of the patients to be in complete or partial remission after 5 years, $19 \%$ to develop end-stage renall disease, about half of which early in the natural history of disease, and $14 \%$ to develop late onset recurrent nephrotic syndrome after partial or complete remission. Thrombo-mbolic episodes occurred in 14\% early in the disease history or after recurrence of nephrotic syndrome. Chronic nephrotic syndrome appeared associated with an enhanced risk for chronic renal insufficiency. In the absence of early chronic renal fallure however, a chronic nephrotic syndrome over 2 years duration carried a good prognosis with complete remission in $66 \%$. The chronic renal insufficiencies could be divided in two subsets: those which develop early in the disease history and are presumably immune-mediated and those which develop late and are not necessarily immune-mediated. Repeat renal biopsies are of required to differentiate one subset from the other:

In this thesis we conclude that renal biopsy at presentation gives important information on the diagnosis and prognosis of non-proteinuric and non-azotaemic haematuria. In early IgA nephropathy several clinical and histological criteria are identified which are independent determinants of renal prognosis. In thin GBM nephropathy, we showed that half of the patients develop hypertension, while in older patients in increased risk on renal insufficiency exist. Unfortunately, which patients will actually develop renal insufficiency is not clear at patient presentation, longer follow-up of preferably larger groups should be undertaken to further elucidate this issue. In membranous glomerullonephritis it appears worthwhile to study methods to determine immune-activity of the disease, preferably without having to resort to invasive procedures. 
Nederlandse samenvatting 
In dit proefschrift worden de resultaten van een studie naar het natururlijk beloop van idiopathische glomerulaire aandoeningen beschreven. Deze studie is het vervolg op een eerder prospectief onderzoek naar de incidentie van idiopathische glomerulonephritiden, dat plaatsvond tussen 1980 en 1985 in een aantal regionale ziekenhuizen in Zuid-Limburg. Alle patiënten die zich tussen 1980 en 1985 presenteerden mel symptomen van een glomerulonephritis werden na nierbiopsie geincludeerd. Nierbiopten werden genomen volgens strikte criteria: recent ontstane nierinsufficièntie (serum creatinine $>300 \mu \mathrm{mol} / \mathrm{l}$ ), recent ontstaan nefrotisch syndroom (proteinurie $>5$ gram/ 24 uur, in de afwezigheid van Bence Jones proteinurie), bij beide indicaties werden biopten binnen zes maanden na patiënt-identificatie genomen. Daamaast werden biopten genomen bij chronische proteinuria (> 0.5 gram/24 uar, in de afwezigheid van hypertensie) en chronische micro- of macroscopische hemanurie met of zonder proteinurie, in de afwezigheid van urologische problematiek. Bij de laatste indicatie-groepen werden biopten genomen na minstens zes maanden klachten, maar binnen twee jaar na patiënt-identificatie. In het huidige proefschrift worden de resultaten weergegeven van all patiënten met hematurie of nefrotisch syndroom, van deze patiënten bedroeg de follow-up 8 tot 14 jaar, gemiddeld 11 jaar.

Omdat nog steeds ruim $20 \%$ van alle patiënten die jaarlijks starten met niervervangende therapie nierinsufficiënt zijn geworden op basis van een idiopathische glomerullonephritis, is het zinvol in deze groep patiënten te proberen nierfunctie-verlies te voorkomen of te vertragen. Dit kan door het geven van immunosuppressieve medicatie, immunomodulerende medicatie of door symptomatische behandeling. De behandeling wordt echter gekenmerkt door onzekerheid over de vraag op welk moment met welk soort therapie gestart moet worden. Kennis van het natuurlijk beloop wan glomerulonephritiden kan bijdragen aan de besluitworming. Het bestuderen van het natuurlijk beloop van glomerulaire aandoeningen is echter vaak gecompliceerd door het feit dat studies slechts voor korte tijd (4 jaar) gefinanciend worden en dat dikwijls patiènten ongeacht de duur van symptomen worden ingeslaten. Veel tot nog toe geidentificeerde risicofactoren woor nierinsufficiëntie bij idiopathische glomerulonephritiden zijn dan ook secundair aan effecten van veroudering, hoge bloeddruk etc. op de nier. In onze studie zijn patiënten geincludeerd vroeg in het natuurlijk beloop; ondat financiering onafhankelijk was konden patiënten gemiddeld 11 jaar gevolgd worden. In de diverse hoofdstukken worden het natuurlijk beloop van hematurie en nefrotisch syndroom toegelicht. 
In hoofdstuk 1 bespreken we de literaturur over hematurie en nephrotisch syndroom, waarbij we met name ingaan op het belang van een nierbiopsie. Als patiënten zich melden met hemanurie moet als eerste onderkend worden of het bloedverlies door urologische afwijkingen, dan wel door een nierafwijking veroorzaakt wordt. Bloed onderzoek (totaal IgA bepaling, circulerende immuuncomplexen) en onderzoek naar de vorm van de rode bloedcellen in de urine is niet voldoende om het onderscheid tussen urologische of andere oorzaken te maken. Met name bij patiënten zich met geissoleerde hematurie - zonder proteinurie presenteren, zijn nierbiopten nodig om de exacte diagnose vast te stellen. In een aantal nierziektes, zoals we aantonen voor IgA nephropathie, kan uit het nierbiopt ook informatie gehaald worden over de prognose van de nierziekte. Als bij nierbiopsie blijkt dat een glomerulonephritis de oorzaak is voor de hematurie ${ }_{3}$ hoeft de patiënt niet onnodig onderworpen te worden aan ingrijpende urologische onderzoeken.

Bij patiënten met een membraneuze glomerulonephritis, de meest voorkomende oorzaak van nefrotisch syndroom bij volwassenen, leveren nierbiopten geen informatie op over de prognose van de patiënt. Wel kan bepaald worden in hoeverre de membraneuze glomerulonephritis immunologisch actief is. Bij kinderen met een nephrotisch syndroom wordt vaak afgezien van een nierbiopt, omdat aangetoond is dat immunosuppressieve medicatie vaak de nierziekte tot rust kan brengen. Bij volwassenen blijkt dit veel moeilijker te zijn, deels omdat een aantal mieraandoeningen - zoals minimale laesie nephropathy - minder voorkomt, deels omdat de glomerulonephritiden minder gevoelig zijn voor prednisolon-behandeling. On deze reden is "blinde" behandeling van volwassenen met immunosuppressieve medicijnen niet gewenst. Uit proefdiermodellen, zoalls diermodellen waarbij naar het beloop van een miertransplantatie wordt gekeken, is bekend dat in het begin van cen nierziekte de afweer verantwoondelijk is voor ziektewerschijnselen, terwijl later in het ziektebeloop miet afweer gerichte reparatie mechanismen verantwoordelijk zjin voor aanhoudende ziekte verschijnselen. Dus, zowel de afweer als reparatie mechanismen kunnen de oorzaak zijn van werslechtering van de ziekte.

In hoofdstuk 2 bespreken we de resultaten van de follow-up wan 49 volwassenen (12 met IgA nephropathie, 13 met dunne GBM nierziekte, 20 met normaal, nierweefsel en 4 met gemengde nieraandoeningen) die zich allen met 6 maanden bestaande geisoleerde hematurie - zonder proteinurie of nierfunctie verlies en met een nomale bloeddruk en normale bevindingen bij urologisch onderzoek 
presenteerden. De gemiddelde duw van de follow-up bedroeg 8 tot 14 jaar, gemiddeld 11 jaar. Aan het einde van de follow-up bleek dat bij alle patiènten met dunne glomerulaire membranen ziekte (GBM) ziekte, IgA nephropathie en normal nierweefsel de nierfunctie stabiel was gebleven, in tegenstelling tot de patiënten met gemengde nieraandoeningen. De hematurie was nog steeds aanwezig bij alle patiënten met dunne GBM ziekte, in vrijwel alle patiënten met IgA nephropathie (twee patiènten bleken in remissie te zijn) en in 7 van de 20 patiënten met normaal nierweefsel. Van deze 7 patiênten bleken er vijf klachten te hebben die pasten bij niersteenlijden, zonder dat in hun urine een te hoog calcium- of urinezuurgehalte werd gevonden. De oorzaak van hematurie hing nauw samen met het verloop van de hematurie: bij glomenulaire afwijkingen bleef de hematurie bestaan, terwijl bij normaal nierweefsel na verloop van tijd - gemiddeld 4 jaar - de hematurie verdween. Zeven patiënten met dunne GBM nierziekte, 5 met IgA nephropathie en 3 patiënten met gemengde nierziekten knegen hoge bloeddruk: hoge bloeddruk kwam bij hen significant veel vaker voor dan bij patiënten met normaal nierweefsel. We concluderen dat bij jonge volwassen met hematurie - zonder eiwit verlies - dat langer dan 4 jaar bestaat, een nierbiopt in $86 \%$ van de patiënten een definitieve diagnose oplevert. Deze patiënten met zogenaamde "lichte" glomerulaire nierontsteking hebben wel een verhoogde kans op hoge bloeddruk. De patiënten die een nierbiopt normaal nierweefsel laten zien, moeten vervolgd worden door de uroloog, omdat vaak niersteenlijden de oorzaak is van het bloedverlies in de urine.

In hoofdstuk 3 gaan we nader in op de ziekte dunne GBM nephropathie. Deze ziekte wordt ook wel benigne familliaire hematurie genoemde en lijkt qua presentatie op een bekende erfelijke nierandoening: de ziekte van Alport. Negentien volwassen patienten met dunne GBM nierziekte werden gedurende 9 tot 15 jaar, gemiddeld 12 jaar, verwolgd. Bijna alle (18 van de 19) patiènten hadden chronische microscopisch hernaturie, terwij] I patiënt met macroscopisch bloedwerlies in de urine had. Bij alle patiënten was middels de nierbiopsie de diagnose dunne GBM nierziekte gesteld. Middels oog- en ooronderzoek werd uitgesloten dat de ziekte van Alport bij deze patiènten bestond: geen van de patiënten klaagde over doofheid, slechtziendheid of had afwijkende bevindingen bij audiografisch onderzoek of oogspiegeling. Bij geen van de patiënten was ten tijde van de eerste bioptie familiaire nierinsufficiêntie bekend. Daarnaast werd in alle nierbiopten van de dunne GBM patiënten met immunofluorescentie het Goodpasture-antigeen aangetoond. Ook herhalingsbiopten, na 11 jaar ziekte beloop genomen, lieten bij ultrastructureel onderzoek geen 
afwijkingen suggestief voor Alport zien. Onze patiënten met dunne GBM nierziekte voldeden dan ook njet aan de criteria voor de ziekte van Alport.

Tijdens de follow-up bleek dat hoge bloeddruk bij dunne GBM patiënten veel vaker voorkwam dan bij patiēnten met normaal nierweefsel $(p=0.048)$, én van de dunne GBM patiènten met hypertensie ontwikkelde later nierfunctie-verslechtering. Vijf dunne GBM patiënten lieten een lichte proteïurie zien van meer dan $250 \mathrm{mg} / 24$ uur; in twee patiënten nam dit eiwit verlies belangrijk toe. Dit wordt vermoedelijk veroorzaakt door focale en segmentale glomerulosclerose. Nierbiopten van dunne GBM patiënten lieten een toename van focale globale glomerulosclerose zien, zeker wanneer dit werd vergeleken met IgA nephroparhie patiënten en normaal nierweefsel patiënten die zich met dezelfde klachten presenteerden. Bij de dunne GBM patiènten, met een normale bloeddruk tijdens het hele ziektebeloop, bleek dat bij GFR metingen een vermindering van de nierfunctie in 3 oudere $(>50$ jaar) patiënten lieten zien. We vermoeden dat het ontstaan van hoge bloeddruk, toename van proteinurie en achteruitgang van de nierfunctie wordt veroorzaakt door de toegenomen glomerulosclerosis, zoals gezien wordt in de eerste nierbiopten. Immers, het feit dat afname in GFR wordt gezien in patiënten met een normale bloeddruk, suggereert dat een renaal probleem verantwoordelijk is voor de nierfunctie-verslechtering en toegenomen focale globalie glomerulosclerosis met tubulus atrofie en interstitièle fibrose lijkt een plausibele oorzaak. Overigens bleek dat, hoewel dit niet bekend was ten tijde van het corspronkelijke nierbiopt, in 4 families 6 oudere eerste graads familieleden aanwezig te zijn met onverklaard nierfalen, het geen suggereert dat op de lange termijn dunne GBM nephropathie aanleiding kan geven tot nierfalen, terwijl op de middellange termijn hoge bloeddruk ontstaat.

In hoofdstuk 4 geven we de follow-up van 27 normotensive volwassenen met idiopathische IgA nephropathie die zich presenteerden met chronische hematurie van minstens 6 maanden en die binnen 2 jaar gebiopteerd zijn. Als controle groepen zijn 17 dunne GBM patiënten, 24 patiènten met normall nierweefsel en 9 patiènten met gemengde glomerullopathieèn gebruikt, die eveneens vanwege chronische hematurie werden gebiopteerd. De gemiddelde follow-up bedroeg 11 jaar, range 8 - 14 jaar. Eerdere studies naar het natuurlijk belloop van en risico factoren voor nierinsufficiëntie bij IgA nephropathie hebben aangetoond dat "hevige" microscopische hematurie, ernst van proteinurie, nierinsufficiëntie ten tijde van de nierbiopsie en onbehandelde hypertensie onafhankelijke risico factoren zijn voor het ontstaan van nierinsufficièntie. In deze studies zijn echter patiënten, die vroeg in het ziektebeloop geïdentificeerd zijn, 
wermengd met patienten die reeds langdurige nierschade hebben ondergaan. Om deze reden hangen diverse risico factoren zoals leeftij, hypertensie, nierinsufficiëntie en emstige proteinurie samen, omdat ze allen secundair zijn aan afname van nierweefsel, histologisch gekenmerkt doot focale globale glomerulosclerose, focale en segmentale glomerulosclerose, interstitièle nephritis, interstitièle fibrose en vasculaire hyalinose. In onze studie werden nierbiopten histologisch gescoord volgens gemodificeerde NIH criteria, oorspronkelijk geformuleerd voor lupus nephritis, in termen van activiteit en chroniciteit. Initielle proteinurie hoger dan 1 gram/24 uur was geassocieerd met een hogere activiteitsindex, het bestaan van extracapillaire laesies en ontstaan van nierinsufficientie. Mesangiale IgG depositie en een hoge chroniciteits-index waren geassocieerd met ontwikkeling van hypertensie. Gedurende de follow-up gingen 2 patiënten met een idiopathisch IgA nephropathie in histologische remissie, 9 patiènten hadden symptomen van progressieve nierziekte zoals hypertensie, toename proteinurie of een combinatie van deze. Drie patiènten ontwikkelden nierinsufficiëntie, hiervan werd 1 patient dialyse-afhankelijk. Circulerende immuncomplexen, IgG-IgA rheumafactoren, leeftijd en geslacht hadden geen invloed op het ziektebeloop. In de multivariate analyse waren een hoge chroniciteit index, persisterende erythrocyturie en mesangiale $\operatorname{IgG}$ depasitie onafhankelijke factoren in het natuurlijk beloop van IgA nephropathie.

We concluderen dat bij patiënten die vroeg in het ziektebeloop van IgA nephropathie geidentificeerd worden erythrocyturie, hoge chroniciteits-score en mesangiale IgG afzetting risicofactoren zijn woor een slechte renale prognose. Het verdwijnen van hematurie is geassocieerd met immunopathologische remissie van IgA nephropathie en met lage activiteits - en chroniciterts scores bij de initiële biopsie.

In hoofdstuk 5 bespreken we de resultaten van de follow-up bij membraneuze glomerulonephritis, de meest voorkomende oorzaak voor nephrotisch syndroom bij volwassenen. Diwerse studies hebben het natuurlijk beloop van iMGN bestudeerd, masr de resultaten van deze studies zijn moeilijk te interpretenen omdat verschillende patiënten populaties bestudeerd zijn, geen informatie over histologische stadiëring wordt gegeven en er weinig patiënten met stadium 1 - en dus vroege ziekte bestudeerd zijn. In onze prospectieve regionale studie werden alle patiënten geincludeerd die tussen 1978 - 1987 gebiopteerd werden vanwege een recent ontstaan nephrotisch syndroom. Vierentwintig patiënten hadden een idiopatische membraneuze glomerulonephritis. Alle patiènten hebben een nephrotisch syndroom (proteìnurie $>5$ gram/24 uur), waren normotensief, hadden een creatinine klaring $>60 \mathrm{ml} / \mathrm{min} / 1.73 \mathrm{~m}^{2}$ 
en hadden stadium 1 histologie met bij immunofluorescentie $\operatorname{IgG}, \mathrm{C} 3$ en C5b-9 afzetting, in de afwezigheid van interstitiele afwijkingen. De incidentie van iMGN ws opvallend constant in de observatie periode en bedroeg 1.2 per $15 \times 10^{6}$ wolwassenen per at risk per jaar. Éénentwintig onbehandelde patiënten werden gevolgd voor 8 tot 14 jaar, gemiddeld 11 jaar. Van deze patiënten was $80 \%$ in remissie (compleet $57 \%$, partieel $23 \%$ ) na 5 jaar, late recidieven van nephrotisch syndroom ontstonden in $19 \%$ van de patiënten en chronische nierinsufficiëntie, vroeg of laat ontstaan, ontstond eveneens in 19\%. Thrombo-embolische verschijnselen bestonden in $14 \%$ van de patienten, vroeg in bet ontstaan van het nephrotisch syndroom of ten tijde van een recidief. De hoogte van initiale proteïnurie had geen invloed op renale prognose. Aanhoudend nephrotisch syndroom van meer dan 2 jaar was geassocieerd met een toegenomen risico op chronische nierinsufficiëntie. In de afwezigheid van vroege nierinsufficiëntie is de prognose van aanhoudend nephrotisch syndroom echter goed met complete remissie in $66 \%$. De chronische nierinsufficiènties kunnen in twee groepen verdeeld worden: vroeg ontstane nierinsufficiëntie, welke waarschijnlijk immuun gemedieerd is en laat ontstane nierinsufficiëntie, die niet noodzakelijkerwijs immuun gemedieerd zijn. Herhalingsbiopten zijn noodzakelijk om de immuun gemedieerde van de niet-immun gemedieerde corzaken te onderscheiden.

In dit proefschrift concluderen we dat nierbiopten belangrijke informatie levert over diagnose en prognose in hematurie. In $\operatorname{IgA}$ nephropathie hebben we enkele klinische en histologische criteria geidentificeerd die onafhankelijke factoren zijn in het ziektebeloop. In dunne GBM nephropathie hebben we laten zien dat de helft van de patiënten hypertensie ontwikkelt, terwijl oudere patiënten meer kans op nierinsufficiëntie lopen. Vooralsnog is onduidelijk welke patiënten met GBM nephropathie nierinsufficiënt zullen worden, hiervoor zijn aanvullende studies van langere duur en in grotere groepen nodig. In membraneuze glomerulonephritis zijn. nierbiopten nodig om de immunologische activiteit van de ziekte vast te stellen, alvorens met immunosuppressieve therapie gestart kan worden. Vooralsnog ontbreken niet-invasieve methodes om immunologische activiteit te onderkennen. 


\section{List of publications}

C.M.G. Nieuwhof, C.A. Gaillard, P.J.C. van Breda Vriesman. Membranous glomerulonephritis Towards tailor-made treatment ?. Neth J Med 1993, 42, 109-111.

E.S. Boon, A.A.. Vrij, C. Nieuwhof, J.A. Noord, E. Zeppenfeldt. Small cell lung cancer with paraneoplastic nephrotic syndrome. Eur Resp Journal 1994, 7, 1192 1193.

GJ. Luyckx, C. Nieuwhof, J. Troost, W. Weber. Parkinsonism in alcohol withdrawal: case report and review of the literature. Clin Neurol Neurosurg, 1995, 97, 336-339

C. Nieuwhof, C. Doorenbos, W. Grave, F. de Heer, P. de Leeuw, E. Zeppenfeldt, P. van Breda Vriesman. A prospective study of the natural history of idiopathic nonproteinuric hematuria. Kidney Int 1996, 49; 222-225.

C. Nieuwhof, F. de Heer, P. de Leeuw, K. Leunissen, P. van Breda Vriesman. Thin GBM mephropathy, premature glomerular obsolescence is associated with hypertension in many and late onset renal insufficiency in some.

Kidney Int 1997, 51, 1596-1601

C. Nieuwhof, M. Kruytzer, P. Frederiks, P. van Breda Vriesman. Chronicity index and mesangial $\mathrm{IgG}$ deposition are risk factors for hypertension and renal failure in early IgA nephropathy. Am J Kid Dis 1998, 31:6, 962-970

C. Nieuwhof, J. Stappers, P. van Breda Vriesman. Wegener's granuloma-like renal lesions and ANCA in subacute bacterial endocarditis. Faseb Journal 6 part I 1992, A1239 nr. 2918 (abstract)

C. Nieuwhof, R. Theunissen, R. Goldschmeding, P. van Breda Vriesman. Wegener"s granuloma-like renal lesions and ANCA in subacute bacterial endocarditis. Nephrol Dial Transpl 1992, 693 (abstract). 
C. Nieuwhof, W. Grave, C. Doorenbos, K.Leunissen, E. Zeppenfeldt, P. van Breda Vriesman. Natural history of idiopathic IgAN and thin GBM disease in adults. Kidney Int 1993, 43, 970 (abstract). 


\section{Curriculum Vitae}

Ciascuno di noi è uno e centamila e cioe, in definitiva, nessino: siano tanti quanti sono coloro che ci osserwano Luigi Pirandello: Uno, nessuno e centomila

The author of this thesis was born June 9, 1964 in Beek, Limburg. After finishing secondary school in Geleen (Athenaeum B, 1982) she immediately started studying Medicine at the Rijksuniversiteit Limburg, from which she graduated in 1988. She continued studying International Relations at the School of Advanced International Studies of the Johns Hopkins University in Bologna, Italy, where she successfully completed the one-year diploma course in 1989. She returned to the Netherlands to work at the Department of Cardiology in Sittard and the Department of Medicine in the University Hospital in Maastricht, before starting to work at the Department of Immunology at the Rijksuniversiteit Limburg in 1990, under supervision of Prof. Dr. Peter van Breda Vriesman. The work performed in this period has been described in this thesis. In 1994 she started training in Medicine at the University Hospital of Maastricht, under Prof. Dr. G. Flendrig and later Prof. Dr. H. Hillen. She will pursue further training in Clinical Immunology. 


\section{Naschrift}

Het is zover, het wordt tijd om afscheid te nemen. Gedurende bijna acht jaren heb ik rondgelopen bij de vakgroep Immunologie te Maastricht, aanvankelijk dagelijks op het lab - zelfs mog op het oude BMC -, later voomamelijk achter de computer. Het waren acht fantastische jaren, waarvoor ik als eerste mijn promotor Peter van Breda Vriesman, onder wiens supervisie dit proefschrift tot stand is gekomen, wil bedanken. Ik heb enorm veel geleerd dankzij jou; over nierziekten, immunologie, wetenschap, geneeskunde en tal van andere zaken: ik zal er zeker vaak en met veel plezier aan blijven denken. Naast de professor wil ik ook zijn twee rechterhanden bedanken, Anne en Anita, voor hun geduld en bereidheid mij uit de brand te helpen op moeilijke momenten. Mijn paranimfen en labgenoten van het eerste uur, Henk en Ruud, wil ik prijzen voor bun verhelderende discussies over de Nederlandse politiek op het lab, waarvan ik na twee minuten de draad al kwijt was. Henk maakt briljante niercoupes en wist, met Zeeuwse nuchterheid, de dingen altijd op de juiste plaats te zetten. Ik heb genoten van de ritjes achter op de motor en hoop nog op een tochtje door de Ardennen. Ruud heeft mij ingewijd in de fijne keuken van de eiwitchemie, ik heb bewondering voor de toewijding waarmee je je werk doet. Met de komst van Lucienne op het lab kreeg ik versterking aan het vrouwelijke front, ik dank je voor de introductie bij het Luikse roeien, nooit gedacht dat dit zou leiden tot een serieuze overweging deel te nemen aan het Belgische kampioenschap lichte dames in de skiff (er waren echter andere prioriteiten). Mijn boot-en kamergenootje Astrid wil ik bedanken voor alle avonturen op en in het water, ik denk dat we hiermee een basis gelegd hebben voor een lange vriendschap, ook al zit jij in Uder en ik op "de Belsj". Mijn andere mede-AlO's, Leo de lange man, Josien, Ywo en Wiel wil ik bedanken voor hun vriendschap, kritische opmerkingen, saté met pinda-saus, ideeèn over multiple toepassingen van een stethoscoop en goede voorbeelden. Harry, het is dat Muis de beer heeft opgegeten, anders had hij er nog gestaan, zó mooi was hij. Ad, Jan, José, Maria en Mia wil ik bedanken voor de gezelligheid in en buiten het lab en vele andere onnoembare zaken. De mensen van het "routine" -immunologie-lab dank ik voor hun efficientie, als ik nog even iets wilde weten of een mooi immunofluorescentie-plaatje wilde zien. Ik hoop nog vaak met jullie te kunnen samenwerken.

Dit proefschrift was niet mogelijk geweest zonder de medewerking van alle patiënten, die mij toelieten in hun huis, met naald en bloedbuis en grote bokalen. Ook 
de nefrologen/internisten van de Regionale Nierwerkgroep Limburg: Karel Leunissen, Bob Zeppenfeldt, Leon Frenken, Hans Wolters, Theo van de Wiel, Cees Doorenbos, Frans de Heer, Willem Grave, Cees van Deursen en Jaap Wals wil ik bedanken voor hun toestemming in om hun dossiers te neuzen en hun patiënten te vervolgen. Er ligt een schat aan informatie in het Regionale Nierarchief begraven en ik hoop dat na mij nog vele mensen aan het delven zullen slaan. Mijn huidige opleider in interne geneeskunde, Harry Hillen, ben ik erkentelijk voor zijn geduld en vertrouwen in mij, Karel Leunissen woor zijn ondersteuning op tijdgebied en Peter de Leeuw voor zijn toestemming gebruik te maken wan de expertise op het hypertensie-lab. Mijn assistenten-maatjes dank ik voor hur sympathie, ik vind dat ik het niet slecht getroffen heb met jullie.

Et enfin toi, Eric, tu seras content que ça c'est fini ! Le pire, c'est que je crains que cela ne changera pas grand chose dans notre vie, parce que j'ai encore tant de projets à faire. Il va falloir que je m'entraine pour la Dent Parrachée, mais ... commençons avec notre maison! Merci pour ta patience, ta compréhension et ton aide, tu as été un chouette copain! Lea, bedankt voor het ontwerp, ik ben blij dat je begreep dat er haast bij was en de tijd ervoor kon vrijmaken. Mijn lieve moeder, broers en zussen, ik denk dat jullie de hoop al hadden opgegeven, maar hier is -ie dan! 